\title{
Teoría de invariantes y variedades tóricas
}

\author{
Autor: Daniel Arturo Sánchez Argáez \\ Departamento de Matemáticas \\ UniVERSidAd AutónOMa MetropolitanA-I \\ Asesor: Dr. Felipe Zaldívar \\ Departamento de Matemáticas \\ UniVERSIDAD AutónOMA METROPOLITANA-I
}





\section{Índice general}

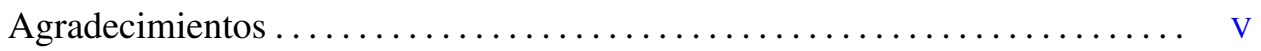

Introducción $\ldots \ldots \ldots \ldots \ldots \ldots \ldots \ldots \ldots \ldots \ldots \ldots \ldots \ldots \ldots \ldots \ldots$ VII

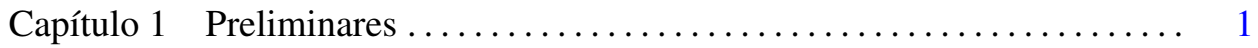

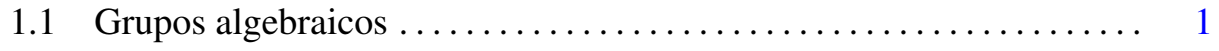

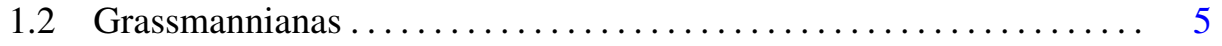

1.3 Variedades de Schubert........................... 12

1.4 Teoría de monomios estándar en variedades de Schubert .......... 13

Capítulo 2 Acciones de grupos algebraicos .................... 19

2.1 Teoremas fundamentales ............................ 24

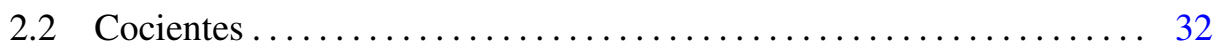

2.3 Cocientes en geometría algebraica $\ldots \ldots \ldots \ldots \ldots \ldots \ldots \ldots \ldots \ldots$

Capítulo 3 Variedades tóricas como cocientes.................. 47

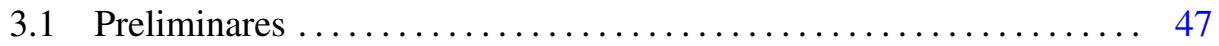

3.2 Variedades tóricas como cocientes $\ldots \ldots \ldots \ldots \ldots \ldots \ldots \ldots \ldots \ldots 2$

Bibliografía......................................... 65 



\section{Agradecimientos}

Este trabajo ha sido realizado bajo la dirección del Dr. Felipe Zaldívar, a quién le extiendo un sincero agradecimiento por toda la paciencia, empatía y enseñanza brindada en estos últimos años, Así mismo agradezco a los sinodales Dr. Pedro Luis del Angel y Dr. Javier Elizondo por sus observaciones y su tiempo.

A mi familia, en toda su extensión, por estar siempre presentes y patrocinar este proyecto de múltiples formas. A quiénes también les ofrezco una disculpa por la tardanza en concluir, en especial a mi Madre. Por todo el cariño que les tengo, les dedico mi trabajo. 



\section{Introducción}

La tesis tiene por objetivo dar la demostración de que toda variedad tórica es un buen cociente de una variedad algebraica por la acción de un grupo algebraico afín, ambos debidamente construidos. El trabajo se divide en tres capítulos.

El capítulo 1 consiste de los preliminares, definiciones y propiedades básicas de grupos algebraicos afines. El contenido de este capítulo fue estudiado de los libros [5], [4] y [8] para la sección 1.1. Las secciones $1.2,1.3$ y 1.4 son requeridas para la demostración de los teoremas fundamentales de la teoría de invariantes.

El capítulo 2 introduce las acciones de grupos algebraicos en variedades afines. Da una demostración de los teoremas fundamentales. También define los tipos de cocientes de una acción de un grupo algebraico en una variedad que permiten construir espacios de órbitas que sigan perteneciendo a la categoría de variedades algebraicas.

En los capítulos 1 y 2 la teoría es desarrollada sobre un campo algebraicamente cerrado.

Por último, el capítulo 3 en su sección 3.1 contiene los propiedades básicas de variedades tóricas así como la notación que será utilizada. Dado que esta área es extensa solamente serán citados los resultados en dicha sección, siendo [1] y [10] las referencias importantes. Otro texto sugerido para profundizar en el tema es [9]. Se concluye la tesis en la sección 3.2 con la demostración de D. Cox en su artículo [2]. En este capítulo se trabaja sobre el campo de los números complejos. 


\section{Capítulo 1}

\section{Preliminares}

\subsection{Grupos algebraicos}

En esta sección se darán las nociones básicas y necesarias para el desarrollo del trabajo. En toda la tesis, $k$ denota un campo algebraicamente cerrado.

Definición 1.1. Sea $G$ una variedad algebraica sobre k, tal que $G$ está dotado de una estructura de grupo abstracto. Si $m: G \times G \rightarrow G$, la operación en el grupo, e i : $\mathrm{G} \rightarrow \mathrm{G}$, el morfismo de inversión, son morfimos algebraicos, entonces diremos que $\mathrm{G}$ es un grupo algebraico. Si $\mathrm{G}$ es una variedad algebraica afín no necesariamente irreducible diremos que $\mathrm{G}$ es un grupo algebraico afín o un grupo algebraico lineal.

Ejemplo 1.2. Sea $\operatorname{Mat}_{n}(k)$, el grupo de matrices de tamaño $n \times n$ con entradas en $k$, se puede identificar con $\mathbb{A}^{n^{2}}$. Su anillo de coordenadas está generado por las entradas de las matrices. El subgrupo de matrices invertibles $\mathrm{GL}_{n}(k) \subset \operatorname{Mat}_{n}(k)$ es un abierto de $\operatorname{Mat}_{n}(k)$, ya que es el complemento del núcleo del morfismo determinante det $: \operatorname{Mat}_{n}(k) \rightarrow k$ puesto que para toda $A \in G_{n}(k)$ la función determinante es diferente de cero. Usando el truco de Rabinowitsch se tiene que

$$
\operatorname{GL}_{\mathfrak{n}}(k)=\left\{A \in \operatorname{Mat}_{\mathfrak{n}}(k) \mid \operatorname{ydet}_{i, j}(A)-1=0\right\} \subset \mathbb{A}^{n^{2}+1}
$$

Entonces $\mathrm{GL}_{\mathfrak{n}}(k)$ es cerrado en $\mathbb{A}^{n^{2}+1}$. Por lo tanto $\mathrm{GL}_{\mathfrak{n}}(k)$ es un grupo algebraico.

Ejemplo 1.3. Del ejemplo anterior se deriva otro muy importante, el caso cuando $\mathrm{n}=1$ nos brinda el grupo multiplicativo $\mathbb{G}_{\mathrm{m}}=\mathrm{k}^{*}$. A su vez podemos definir otro grupo algebraico de gran interés en el capítulo 3. Un toro algebraico es una variedad algebraica $T$ isomorfa a $\left(k^{*}\right)^{n}$.

Como ejemplos de grupos algebraicos tenemos los grupos finitos, el grupo lineal general $\mathrm{GL}_{n}(k)$, el grupo lineal general proyectivo $\mathrm{PGL}_{n}(k)$, el grupo lineal especial $\mathrm{SL}_{n}(k)$, el grupo lineal especial proyectivo $\operatorname{PSL}_{n}(k)$.

Algunas propiedades que se pueden observar de los grupos algebraicos afines es que la traslación por un elemento $g \in G, \lambda_{g}: G \rightarrow G$ dada por $\lambda_{g}(h)=g \cdot h$ es un morfismo entre variedades con inversa $\lambda_{g^{-1}}$ por lo tanto es un isomorfismo. Esto nos permite obtener propiedades geométricas en un punto y trasladarlas a todo punto en $G$. Esto nos garantiza que $G$ es una variedad lisa puesto que $G$ contiene puntos lisos y por el isomorfismo de traslación se deduce la no singularidad de G. Para precisar esto, sea $S$ el conjunto de puntos lisos de $G$, el cuál sabemos que es distinto del vacío. Ahora bien, sea $x \in S$ y $g \in G$ entonces $\lambda_{g x^{-1}}(x)=g x^{-1} \chi=g$ demostrando así que todo pundo de $\mathrm{G}$ es liso. 
Es necesario definir lo que es un morfismo entre grupos algebraicos.

Definición 1.4. Sean $\mathrm{G}$ y $\mathrm{H}$ dos grupos algebraicos afines. Un morfismo entre grupos algebraicos $\phi: \mathrm{G} \rightarrow \mathrm{H}$ es un morfismo entre variedades que a su vez es un homomorfismo de grupos. Es decir, respeta la estructura subyacente.

Al igual que en la teoría de grupos lo natural es estudiar los subconjuntos que heredan la estructura de grupo algebraico.

Definición 1.5. Sea $\mathrm{G}$ un grupo algebraico afín. Un subgrupo abstracto $\mathrm{H} \subset \mathrm{G}$ tal que $\mathrm{H}$ es cerrado (en la topología de Zariski) se llamará un subgrupo algebraico afín

Retomando el ejemplo de $\mathrm{GL}(\mathrm{n}, \mathrm{k})$ podemos obtener los siguientes subgrupos:

\section{Ejemplo 1.6.}

(a). El subgrupo abstracto $B(n, k) \subset \mathrm{GL}(n, k)$ de matrices triangulares superior invertibles. Para verificar que es un cerrado de $\operatorname{GL}(n, k)$ se requiere que $B(n, k)$ sea los ceros de un conjunto de polinomios, para ello sólo observemos que $B(n, k)=V\left(x_{i, j} \mid x_{i, j}=0\right.$ si $\left.j<i\right)$.

(b). El subgrupo abstracto $D(n, k) \subset G L(n, k)$ de las matrices diagonales invertibles. Es claro que es un cerrado de $\mathrm{GL}(n, k)$ ya que son los ceros $V\left(\left\{x_{i, j} \mid i \neq j\right\}\right)$.

Obsérvese que todo elemento $B \in B(n, k)$ se puede escribir como el producto de un elemento $D \in D(n, k)$ por un elemento $A \in \operatorname{Mat}(n, k)$, donde $A$ satisface que

$$
x_{i, j}=0 \quad \text { si } \quad j \leqslant i .
$$

Realizando un conteo de las entradas de $A$ que podrían ser diferentes de cero obtenemos que hay $\frac{n(n-1)}{2}$ de ellas. Identificando toda matriz que cumpla $(*)$ con $\mathbb{A} \frac{n(n-1)}{2}$. Con ello podemos pensar que $B(n, k)$ es isomorfo a $D(n, k) \times \mathbb{A}^{\frac{n(n-1)}{2}}$, dado que $\operatorname{dim} D(n, k)=n y \operatorname{dim} \mathbb{A}^{\frac{n(n-1)}{2}}=\frac{n(n-1)}{2}$ se sigue que $\operatorname{dim} B(n, k)=$ $\frac{\mathrm{n}(\mathrm{n}+1)}{2}$.

Como grupo abstracto, $G$ puede tener muchos subgrupos sin embargo no todos son subgrupos algebraicos. Se define el $Z(G)=\{x \in G \mid x y=y x$ para todo $y \in$ G como el centro de G. Si definimos $\psi_{y}=m \circ\left(\lambda_{y}, \lambda_{y^{-1}} \circ i\right) \circ \Delta: G \rightarrow$ $\mathrm{G}$ en donde $\mathrm{m}$, i y $\lambda$ son los morfimos de multiplicación, inversión y traslación respectivamente, y $\Delta$ el morfismo diagonal se tiene que $\psi_{y}$ es continua puesto que es composición de funciones continuas. Ahora si $x \in \mathrm{G}$, entonces

$$
\begin{aligned}
\psi_{y}(x) & =m \circ\left(\lambda_{y}, \lambda_{y^{-1}} \circ i\right) \circ \Delta(x) \\
& =m \circ\left(\lambda_{y}, \lambda_{y^{-1}} \circ i\right)(x, x) \\
& =m \circ\left(y x, y^{-1} x^{-1}\right) \\
& =y x \cdot y^{-1} x^{-1}
\end{aligned}
$$


Ahora bien, $\psi_{y}^{-1}(e)$ es el conjunto de elementos que conmutan con y y es cerrado por ser $\psi_{y}$ continua. Recorriendo sobre todos los elementos $y \in G$ se tiene que:

$$
Z(G)=\bigcap_{y \in G} \psi_{y}^{-1}(e)
$$

Por lo tanto Z(G) es cerrado y así es un subgrupo algebraico.

Otro concepto que surge es el relacionado con los subgrupos normales abstractos. Se dirá que un subgrupo algebraico es normal si es normal como subgrupo abstracto.

En los siguientes lemas se darán condiciones para obtener subgrupos algebraicos.

Lema 1.7. Sea $\mathrm{G}$ un grupo algebraico afín, $U$ y $\mathcal{V}$ subconjuntos abiertos no vacíos de $\mathrm{G}$ con $\mathcal{V}$ denso. Entonces $\mathrm{G}=\mathcal{U} \cdot \mathcal{V}$

Demostración. Aplicando los isomorfismo de traslación e inversión entre variedades algebraicas se tiene que para toda $x$ en $\mathrm{G}$ el conjunto $x \mathcal{V}^{-1}=\left\{x v^{-1} \mid \mathcal{V} \in\right.$ $\mathcal{V}\}$ es un abierto y denso en $G$. Por lo tanto $x \mathcal{V}^{-1} \cap \mathcal{U} \neq \emptyset$ entonces existe $a \in \mathcal{U}$ y $\mathrm{b} \in \mathcal{V}$ tal que $x \mathrm{~b}^{-1}=\mathrm{a}$ es decir, $x=\mathrm{ab}$ quedando así demostrado el lema.

Lema 1.8. Sea $\mathrm{G}$ un grupo algebraico afín y $\mathrm{H}$ un subgrupo abstracto. Entonces $\overline{\mathrm{H}}$ es un subgrupo algebraico afín.

Demostración. Dado que $\mathrm{H}$ es un subgrupo de $\mathrm{G}$ cumple que $\mathrm{m}(\mathrm{H} \times \mathrm{H}) \subseteq$ $\mathrm{H}$, entonces

$$
m(\overline{\mathrm{H}} \times \overline{\mathrm{H}})=\mathrm{m}(\overline{\mathrm{H} \times \mathrm{H}}) \subseteq \overline{\mathrm{H}}
$$

por lo tanto es un subgrupo algebraico.

Lema 1.9. Sea $\mathrm{G}$ un grupo algebraico afín y $\mathrm{H}$ un subgrupo abstracto que es construible en $\mathrm{G}$. Entonces $\mathrm{H}$ es un subgrupo algebraico afín.

Demostración. Existe $\mathcal{U} \subset \mathrm{H}$ abierto y denso en $\overline{\mathrm{H}}$. Sea $h \in \mathcal{U}$ entonces existe $h^{-1} \in \mathrm{H}$ y $h \cdot \mathcal{U}$ contiene a la identidad, denotemos por $\mathcal{V}=h^{-1} \mathcal{U}$, el cual es un abierto y denso en $\overline{\mathrm{H}}$ por el isomorfismo de traslación. Ahora bien, para toda $g \in \mathrm{H}$ se cumple que $\mathrm{g} \cdot \mathcal{V} \subset \mathrm{H}$ por lo que $\bigcup_{\mathrm{g} \in \mathrm{H}} \mathrm{g} \cdot \mathcal{V} \subset \mathrm{H}$. Por otro lado, dado que la identidad está en $\mathcal{V}$ se tiene la otra contención. Por lo tanto $\mathrm{H}=\bigcup_{\mathrm{g} \in \mathrm{H}} \mathrm{g} \cdot \mathcal{V} \subset \mathrm{H}$ es un abierto en $\overline{\mathrm{H}}$ y denso, aplicando el lema 1.6

$$
\overline{\mathrm{H}}=\mathrm{H} \cdot \mathrm{H}=\mathrm{H}
$$

Por lo tanto $\mathrm{H}$ es un subgrupo algebraico.

Como variedad algebraica afín, podemos analizar las componentes irreducibles de $G$ y recordando que es un espacio noetheriano solamente existen un número finito de componentes irreducibles. Entonces el elemento identidad del grupo, e, está contenida en al menos una de ellas. El siguiente lema nos garantiza la unicidad de dicha componente y unas propiedades de la conexidad de $\mathrm{G}$ 
Proposición 1.10. Sea G un grupo algebraico afín, entonces se cumplen las siguientes propiedades

(a). Existe una única componente irreducible que contiene a la identidad e del grupo, denotémosla como $\mathrm{G}_{e}$.

(b). $\mathrm{G}_{e}$ es un subgrupo normal de $\mathrm{G}$ de índice finito en $\mathrm{G}$ cuyas clases laterales son las componentes conexas e irreducibles de $\mathrm{G}$.

(c). Cada subgrupo cerrado de índice finito en $\mathrm{G}$ contiene a $\mathrm{G}_{e}$.

(d). Si $\mathrm{G}$ y $\mathrm{H}$ son grupos algebraicos afines y $\phi: \mathrm{H} \rightarrow \mathrm{G}$ es un morfismo entre grupos algebraicos, entonces $\phi\left(\mathrm{H}_{e}\right)=\phi(\mathrm{H})_{e}$

Demostración. Sean $Y_{1}, \ldots, Y_{m}$ las componentes irreducibles que contienen a e. Por la irreducibilidad de $Y_{i}$ se tiene la irreducibilidad del producto $\mathrm{Y}=\mathrm{Y}_{1} \times \ldots \times \mathrm{Y}_{\mathrm{m}}$. Definimos el morfismo:

$$
\begin{gathered}
\theta: Y \longrightarrow Y_{1} \cdots Y_{m} \subset G \\
\left(y_{1}, \ldots, y_{m}\right)=y_{1} \cdots y_{m} \in G
\end{gathered}
$$

Esto implica que $Y_{1} \ldots Y_{m}$ es irreducible en $G$ por lo tanto existe $i$ tal que $Y_{1} \ldots Y_{m} \subset Y_{i}$. Por otro lado para toda $i$ se tiene que $Y_{i} \subset Y_{1} \ldots Y_{m}$. Lo cual implica que $m=1$

Denotemos por $G_{e}$ la componente irreducible de $G$ que contiene a e. Llamaremos a $G_{e}$ la componente de la identidad de $G$.

(b): Para todo $x \in G_{e}$ se tiene que $x^{-1} G_{e}$ es una componente irreducible de $G$ que tiene a la unidad, esto es consecuencia de que la traslación por un elemento de $G$ es un isomorfismo de $G$ en $G$. Por lo que $G_{e}=\left(G_{e}\right)^{-1}$. Ahora bien $\mathrm{G}_{e} \mathrm{G}_{e}=\mathrm{G}_{e}\left(\mathrm{G}_{e}\right)^{-1}=\mathrm{G}_{e}$ por lo tanto $\mathrm{G}_{e}$ es un subgrupo. Ahora sea $x \in \mathrm{G}$ aplicando de nuevo el isomorfismo de traslación a ambos lados de $G_{e}$ es decir $x G_{e} \chi^{-1}$ se tiene de nuevo que es una componente irreducible que contiene a la unidad, por lo tanto debe de ser igual a $\mathrm{G}_{e}$ probando con ello que la componente de la identidad es un subgrupo normal en $\mathrm{G}$. Por último recordemos que las clases laterales de $G$ por $G_{e}$ son las traslaciones de $G_{e}$ y por el análisis de las lineas anteriores se tiene que toda clase lateral es una componente irreducible, observemos que dado que $G$ es una variedad afín se tiene que es un espacio noetheriano por lo que solamente existe una cantidad finita de componentes irreducibles, por lo tanto $G_{e}$ es de índice finito. Recordemos que las clases laterales inducen una partición en $\mathrm{G}$ por lo que todas las componentes irreducibles son disjuntas y a su vez conexas. Cada conjunto conexo intersecta solamente a una componente conexa, de aquí que cada componente conexa es unión de componentes irreducibles. Sin pérdida de generalidad, consideremos a $G_{e}$, entonces existe una componente conexa $C_{e}$ tal que $\mathrm{G}_{e} \subset \mathrm{C}_{e}$, si la contención es propia, se tiene que el complemento es unión finita de componentes irreducibles, puesto que $\mathrm{G}_{e}$ es de índice finito. Considerando la topología relativa en $C_{e}$ se tiene que $G_{e}$ es abierto, por otro lado, $G_{e}$ es cerrado implicando que su complemento sea abierto, con esto hemos construido una separación de $\mathrm{C}_{e}$ contradiciendo el hecho de que sea conexo. Con ello se demuestra que $\mathrm{G}_{e}=\mathrm{C}_{e}$ y por el isomorfismo de traslación se concluye que las componentes conexas coinciden con las componentes irreducibles. 
(c) Sea $\mathrm{H}$ un subgrupo cerrado de índice finito en $\mathrm{G}$, es decir existe un número finito de clases laterales de $\mathrm{H}$, denotemoslas por $\mathrm{H}_{i}$, de nuevo, por el isomorfismo de traslación estas clases laterales son cerradas. Consideremos $V=\bigcup_{i=1}^{k} H_{i}$ de las clases laterales distintas a $\mathrm{H}, \mathrm{V}$ es cerrado, por lo que su complemento es abierto, es decir $\mathrm{H}$. Considerando $\mathrm{H}_{e}=\mathrm{H} \cap \mathrm{G}_{e}$ con la topología relativa se tiene que $\mathrm{H}_{e}$ es cerrado y abierto. Considerando a $\mathrm{H}_{e}$ como abierto se tiene que su complemento es cerrado, con lo cual hemos obtenido una partición de $\mathrm{G}_{e}$ en cerrados disjuntos cuyo unión es $\mathrm{G}_{e}$ contradiciendo su condición de irreducibilidad. Por lo tanto $\mathrm{H}_{e}=\mathrm{G}_{e}$ es decir, $\mathrm{G}_{e} \subset \mathrm{H}$.

(d) Dado que $\mathrm{H}_{e}$ es de índice finito en $\mathrm{H}$ se tiene que $\varphi\left(\mathrm{H}_{e}\right)$ es índice finito en $\varphi(\mathrm{H})$. Dado que $\varphi$ es continua, $\varphi\left(\mathrm{H}_{e}\right)$ es irreducible porque es la imagen de una componente irreducible. Como $\varphi\left(\mathrm{H}_{e}\right)$ contiene a la identidad implica que $\varphi\left(\mathrm{H}_{e}\right) \subseteq \varphi(\mathrm{H})_{e}$. Ahora bien, por el inciso anterior se tiene que $\varphi(\mathrm{H})_{e} \subseteq \varphi\left(\mathrm{H}_{e}\right)$. Por lo tanto $\varphi\left(\mathrm{H}_{e}\right)=\varphi(\mathrm{H})_{e}$.

Corolario 1.11. Sea $\mathrm{G}$ un grupo algebraico afin y $\varphi: \mathrm{G} \rightarrow \mathrm{G}$ es un automorfismo de $\mathrm{G}$. Sea $\mathrm{H} \subset \mathrm{G}$ un subgrupo cerrado tal que $\varphi(\mathrm{H}) \subset \mathrm{H}$. Entonces $\varphi(\mathrm{H})=\mathrm{H}$.

Demostración. Dado que $\mathrm{H}$ es un subgrupo cerrado, $\mathrm{H}$ es un grupo algebraico, entonces la restricción de $\varphi$ a $\mathrm{H}$ es un morfismo entre variedades. Ahora bien, supongamos primero que $\mathrm{H}$ es conexo, entonces $\varphi(\mathrm{H})$ es conexo y aplicando el último inciso del lema anterior se obtiene:

$$
\varphi(\mathrm{H})=\varphi\left(\mathrm{H}_{e}\right)=\varphi(\mathrm{H})_{e} \subset \mathrm{H}
$$

Como $\varphi$ es automorfismo, es un isomorfismo entre las componentes irreducibles de $\mathrm{H}$ y $\varphi(\mathrm{H})$, es decir, un isomorfismo entre $\mathrm{H}$ y $\varphi(\mathrm{H})$, por lo tanto $\mathrm{H}=\varphi(\mathrm{H})$.

Supongamos que $\mathrm{H}$ no es conexo, sea $\mathrm{H}_{e}$ la componente irreducible que contiene a la identidad, $\mathrm{H}_{e}$ es de índice finito en $\mathrm{H}$ y $\varphi(\mathrm{H})_{e}$ también es de índice finito en $\varphi(\mathrm{H})$. Por el inciso $d$ del lema anterior, se tiene que $\varphi\left(\mathrm{H}_{e}\right)=\varphi(\mathrm{H})_{e}$. Por hipótesis $\varphi\left(\mathrm{H}_{e}\right) \subset \mathrm{H}$ y dado que $\varphi\left(\mathrm{H}_{e}\right)$ es un conexo que contiene a la identidad se tiene que $\varphi\left(\mathrm{H}_{e}\right) \subset \mathrm{H}_{e}$ y por el primer caso concluimos que $\varphi\left(\mathrm{H}_{e}\right)=\mathrm{H}_{e}$. Lo cual implica que las clases laterales de $\mathrm{H}$ у $\varphi(\mathrm{H})$ están en correspondencia, es decir, sean $\left\{g_{1} \mathrm{H}_{e}, g_{2} \mathrm{H}_{e}, \ldots, g_{s} \mathrm{H}_{e}\right\}$ las clases laterales en $\mathrm{H}$ por $\mathrm{H}_{e}$, por definición $\mathrm{g}_{\mathrm{i}} \notin \mathrm{H}_{e}$, para $i>1$ por lo que $\varphi\left(\mathrm{g}_{\mathrm{i}}\right) \notin \varphi(\mathrm{H})_{e}$ para toda $i$. Por otro lado $\varphi$ es un isomorfismo, por tanto, $g_{i} H_{e} \simeq \varphi\left(g_{i}\right) \varphi\left(H_{e}\right)$ para toda $i$. Por lo que

$$
\mathrm{H}=\bigcup_{i=1}^{s} g_{i} H_{e} \simeq \bigcup_{i=1}^{s} \varphi\left(g_{i} H_{e}\right)=\bigcup_{i=1}^{s} \varphi\left(g_{i}\right) \varphi(H)_{e}=\varphi(H) .
$$

Finalmente, por hipótesis, $\varphi(H) \subset H$, por lo tanto, $H=\varphi(H)$.

\subsection{Grassmannianas}

Esta sección y la siguiente tienen por objetivo dar las bases para la demostración de los teoremas fundamentales de la teoría de invariantes. Al poder definir las variedades de Schubert, $V_{[i]}$ en las Grassmannianas $G(r, n)$ podremos llevar a cabo una elección adecuada de $i$ y $r, n$ y demostrar que los teoremas fundamentales se siguen de manera directa. 
Sea $V$ un espacio vectorial de dimensión finita, sobre el campo $k, \operatorname{dim}_{k} V=n$. Para cualquier entero fijo $r$ tal que $1 \leqslant r<n$ tenemos:

Definición 1.12. La Grassmanniana, $G(r, n)$ se define como el conjunto de todos los subespacios lineales $\mathrm{U}$ de $\mathrm{V}$ tales $\operatorname{dim}_{k} \mathrm{U}=\mathrm{r}$,

$$
\mathrm{G}(\mathrm{r}, \mathrm{n})=\left\{\mathrm{U} \subset \mathrm{V} \mid \operatorname{dim}_{\mathrm{k}} \mathrm{U}=\mathrm{r}\right\} .
$$

Sea $U$ un elemento de $G(r, n)$ y $a_{1}, \cdots, a_{r}$ una base de $U$ donde $a_{j}$, expresado en la base canónica $e_{1}, \cdots, e_{n}$ de $V$, se puede escribir como vector columna de la siguiente manera:

$$
\left(\begin{array}{c}
a_{1, j} \\
a_{2, j} \\
\vdots \\
a_{n, j}
\end{array}\right) \text { con } a_{i, j} \quad \text { en } k \text { para } 1 \leqslant i \leqslant n \quad y \quad 1 \leqslant j \leqslant r .
$$

Por lo que podemos asignar a cada elemento de $G(r, n)$ una matriz $A$ de tamaño $n \times r$ cuyo rango es igual a $r$. Si $a^{\prime}{ }_{1}, \cdots, a^{\prime}{ }_{r}$ es otra base de $U$ y $A^{\prime}$ la matriz asociada de tamaño $n \times r$, dado que ambas representan el mismo subespacio vectorial, existe $C$ en $\mathrm{GL}_{r}(\mathrm{k})$, un cambio de base tal que $A^{\prime}=A C$. Por otro lado, dadas dos matrices $A$ y $A^{\prime}$ de rango $r$, tales que existe $C \in \mathrm{GL}_{\mathrm{r}}(\mathrm{k})$ cumpliendo que $A^{\prime}=A C$, entonces $A$ y $A^{\prime}$ generan el mismo subespacio vectorial y las columnas de ambas matrices son linealmente independientes (puesto que tienen $r$ columnas). Entonces podemos identificar las matrices de tamaño $n \times r$ como un punto de $\mathbb{A}^{\mathrm{nr}}$, lo cual nos permite dar la siguiente identificación:

$$
\mathrm{G}(\mathrm{r}, \mathrm{n})=\left(\mathbb{A}^{\mathrm{nr}} \backslash \mathrm{Z}\right) / \sim
$$

donde $Z$ es el conjunto de matrices de rango menor estrictamente que $r$ y la relación descrita previamente, es decir, dos matrices $A$ y $A^{\prime}$ en $G(r, n)$, están relacionadas, $A \sim A^{\prime}$, si y sólo si existe $C \in G L_{r}(k)$ tal que $A^{\prime}=A C$. Es decir, tenemos una acción,

$$
\begin{aligned}
\left(\mathbb{A}^{n r} \backslash Z\right) \times G(k) & \longrightarrow\left(\mathbb{A}^{n r} \backslash Z\right) \\
(A, C) & \longmapsto A C
\end{aligned}
$$

siendo $\mathrm{G}(\mathrm{r}, \mathrm{n})$ el espacio de órbitas, es preciso aclarar que todo este análisis ha sido en el sentido conjuntista, conforme al desarrollo de las siguientes secciones se dotará a la Grassmanniana de estructura de variedad algebraica. Para ello definiremos un orden parcial en un conjunto de índices.

Definición 1.13. Definimos el siguiente conjunto

$$
I_{r, n}:=\left\{\underline{i}=\left(i_{1}, i_{2}, \cdots, i_{r}\right) \mid \quad 1 \leqslant i_{1}<i_{2}<\cdots<i_{r} \leqslant n \quad \text { con los } \quad i_{j} \text { enteros }\right\}
$$

Se define un orden parcial, $\leqslant$, en $\mathrm{I}_{\mathrm{r}, \mathrm{n}}$ mediante:

$$
\underline{i} \geqslant \underline{j} \Longleftrightarrow i_{t} \geqslant j_{t} \quad \forall t, \quad 1 \leqslant t \leqslant r .
$$


Sea $N=\# I_{r, n}=\left(\begin{array}{l}n \\ k\end{array}\right)$ y consideremos $\wedge^{r} V \simeq \mathbb{A}^{N}$, espacio que será indexado por $I_{r, n}$, y para cualquier $v$ en $\mathbb{A}^{N}$ distinto de cero, denotemos por $[v]$ en $\mathbb{P}^{N-1}$ el punto determinado por $v$. Sea

$$
\mathrm{X}=\underbrace{\mathrm{V} \oplus \cdots \oplus \mathrm{V}}_{\mathrm{r} \text {-veces }}=\mathbb{A}^{\mathrm{rn}}
$$

aplicando el producto exterior:

$$
\begin{aligned}
\wedge^{r}: X & \longrightarrow \wedge^{r} \vee \\
\left(a_{1}, \cdots, a_{r}\right) & \longmapsto a_{1} \wedge \cdots \wedge a_{r}
\end{aligned}
$$

al elegir una base para $\wedge^{r} V$ dada por los elementos $e_{\underline{i}} \operatorname{con} \underline{i} \in I_{r, n}$ podemos identificar a $\wedge^{r} \vee$ con $\mathbb{A}^{N}$ por lo que la $\underline{i}$-coordenada de $a_{1} \wedge \cdots \wedge a_{r}$ es el determinante del $r$-menor de la matriz $A=\left(a_{i, j}\right)$ (cuyas columnas son los vectores $a_{1}, \cdots, a_{r}$ ), formado por las filas $i_{1}, \cdots, i_{r}$. Observese que $a_{1} \wedge \cdots \wedge a_{r}=\overline{0}$ si y sólo si todas sus entradas son cero, lo cual sucede si sólo si el determinante de cada $r$-menor es igual a cero, es decir, si y sólo si $A$ tiene rango menor estricto a $r$. Por lo que si consideramos los puntos de $X$ tales que $a_{1} \wedge \cdots \wedge a_{r}$ son distintos de cero, estamos restringiendo el producto exterior a los subespacios de rango $r$. Ahora bien, la acción de $G L_{r}(k)$ en $\mathbb{A}^{n r}$ es compatible con $\wedge^{r}$. Para visualizar esto, sean $A^{\prime}$ y $A$ dos matrices en la misma órbita, es decir existe $C \in G$ tal que $A^{\prime}=A C$, sean $\left\{a_{1}^{\prime}, \cdots, a_{r}^{\prime}\right\}$ y $\left\{a_{1}, \cdots, a_{r}\right\}$ los vectores columna de cada matriz respectivamente, expresando cada vector columna de $A^{\prime}$ en términos del producto $A C$, tenemos que, para toda $j$

$$
a_{j}^{\prime}=\sum_{i=1}^{r} c_{i, j} a_{i}
$$

siendo $c_{i, j}$ las entradas de la matriz $C$, al definir $\wedge^{r}(A)=\left(a_{1} \wedge \cdots \wedge a_{r}\right)$ tenemos que

$$
\begin{aligned}
\wedge^{r}\left(A^{\prime}\right) & =\wedge^{r}(A C)=\left(\sum_{i=1}^{r} c_{i, 1} a_{i} \wedge \cdots \wedge \sum_{i=1}^{r} c_{i, r} a_{i}\right)=\operatorname{det} C \cdot\left(a_{1} \wedge \cdots \wedge a_{r}\right) \\
& =\operatorname{det} C \cdot \wedge^{r}(A)
\end{aligned}
$$

esta última igualdad es el resultado de aplicar las propiedades de alternancia y linealidad. Por lo que si dos matrices $A, A^{\prime}$ son equivalentes, es decir existe $C \in$ $G L_{r}(k)$ tal que $A^{\prime}=A C$, entonces $\wedge^{r}\left(A^{\prime}\right)$ y $\wedge^{r}(A)$ sólo difieren por un escalar, siendo precisos, por det $C$. Entonces al considerar a $G(r, n)$ como el espacio de órbitas de dicha acción, la siguiente aplicación queda bien definida:

$$
\begin{aligned}
p: G(r, n) & \longrightarrow \mathbb{P}\left(\wedge^{r} V\right)=\mathbb{P}^{N-1} \\
p(U) & \longmapsto\left(p_{\underline{i}}\right)_{\underline{i} \in I_{r, n}}
\end{aligned}
$$

siendo $p_{\underline{i}}$ el determinante del menor de tamaño $r$, de la matriz $A=\left(a_{i, j}\right)$ asociada al subespacio vectorial $U$, formado por las filas $i_{1}, \cdots, i_{r}$, denotemosla por $A_{\underline{i}}$. A esta aplicación se le llama la aplicación de Plücker y a las $p_{\underline{\underline{i}}}^{\prime} \mathrm{s}$ las coordenadas de Plücker. 
Sea $U$ un elemento de $G(r, n)$ y $A=\left(a_{i, j}\right)$ una matriz de tamaño $n \times r$ que lo representa. Si $\underline{l}=\left(l_{1}, \cdots, l_{n}\right)$ es una elección de filas de $A$, no perteneciente a $\mathrm{I}_{\mathrm{r}, \mathrm{n}}$, entonces $\underline{l}$ es de la siguiente forma:

- $\underline{l}$ tiene dos filas iguales, por lo propiedad del determinante, $\underline{l}$ tiene determinante igual a cero, $p_{\underline{\underline{l}}}(\mathrm{U})=\operatorname{det}\left(A_{\underline{\mathbf{l}}}\right)=0$.

- Las filas de $\underline{l}$ no están en orden creciente. Entonces existe $\sigma$ en el conjunto de permutaciones $\{1,2, \cdots, r\}$ tal que $p_{\underline{l}}=(-1)^{\operatorname{sgn} l} p_{\sigma(l)}$ donde $\sigma(\underline{l})=$ $\left(l_{\sigma(1)}, \cdots, l_{\sigma(r)}\right)$ y $l_{\sigma(1)}<l_{\sigma(2)}<\cdots<l_{\sigma(r)}$.

Así, basta considerar solamente los índices de $I_{r, n}$ ya que cualquier elección de $r$-filas distintas se puede ver como un elemento de $I_{r, n}$.

Una base para $\wedge^{r} V$ está dada por $\left\{e_{\underline{i}} \underline{i} \in I_{r, n}\right\}$ donde $e_{\underline{i}}$ es el subespacio generado por la matriz cuyas entradas son $a_{i_{j}, j}=1$ y cero en el resto de ellas. La aplicación de Plücker en algún elemento de la base cumple que

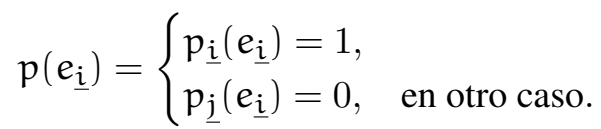

Ahora bien, la importancia de la aplicación de Plücker es la de ser una aplicación inyectiva, lo cual permitirá definir una inmersión cerrada de $G(r, n)$ en un espacio proyectivo adecuado.

Teorema 1.14. La aplicación de Plücker es inyectiva.

Demostración. Sean $\mathrm{U}$ y $\mathrm{U}^{\prime}$ dos elementos de $\mathrm{G}(\mathrm{r}, \mathrm{n})$ tales que $\mathrm{p}(\mathrm{U})=$ $p\left(U^{\prime}\right)$, dado que $\operatorname{Im}(p) \subset \mathbb{P}^{N-1}$ existe una entrada, digamos $\underline{l}=\left(l_{1}, \cdots, l_{r}\right)$ tal que $p_{\underline{\underline{l}}}(\mathrm{U})=p_{\underline{\underline{l}}}\left(\mathrm{U}^{\prime}\right) \neq 0$, para facilitar los cálculos podemos asumir que $p_{\underline{\underline{l}}}(\mathrm{U})=p_{\underline{\underline{l}}}\left(U^{\prime}\right)=1$. Ahora bien, sean $B$ y $B^{\prime}$ las matrices que representan a $\mathrm{U} y$ $\bar{U}^{\prime}$ respectivamente, entonces $p_{\underline{l}}(U)=\operatorname{det}\left(B_{l_{1}, \cdots, l_{r}}\right)=1=\operatorname{det}\left(B_{l_{1}, \cdots, l_{r}}^{\prime}\right)=$ $p_{\underline{l}}\left(U^{\prime}\right)$. Recordemos que $G(r, n)$ es un espacio de órbitas, entonces $B$ y $B C$, siendo $C=B_{l_{1}, \cdots, l_{r}}^{-1}$ en $G_{L_{r}}(k)$, pertenecen a la misma órbita, ahora el menor $(B C)_{l_{1}, \cdots, l_{r}}$ es la matriz identidad en $G_{L_{r}}(k)$ por lo que desde un inicio podemos considerar que la matriz $A=B C$ en donde $A_{l_{1}, \cdots, l_{r}}$ es la matriz identidad. Este mismo análisis es válido para $A^{\prime}=B^{\prime} C^{\prime}$ y $A_{l_{1}, \cdots, l_{r}}^{\prime}$ la matriz identidad. Por lo que podemos considerar a $A$ y $A^{\prime}$ como las matrices que representan a $\mathrm{U}_{\mathrm{y}} \mathrm{U}^{\prime}$ respectivamente. Apliquemos la regla de Cramer (considerando filas) para las entradas de $A$, las entradas de la $i$-ésima fila de $A$ se obtiene por el determinante de la matriz resultante al sustituir en cada columna $j$ de $C$ la fila $i$ es decir

$$
a_{i, j}=\operatorname{det}\left(A_{l_{1}, \cdots, l_{j-1}, i, l_{j+1}, \cdots, l_{r}}\right)
$$

ahora, usando la igualdad en la coordenada de Plücker $p_{l_{1}, \cdots, l_{r}}$ tenemos

$$
\begin{aligned}
a_{i, j} & =\operatorname{det}\left(A_{l_{1}, \cdots, l_{j-1}, i, l_{j+1}, \cdots, l_{r}}\right)=p_{l_{1}, \cdots, l_{j-1}, i, l_{j+1}, \cdots, l_{r}}(U) \\
& =p_{l_{1}, \cdots, l_{j-1}, i, l_{j+1}, \cdots, l_{r}}\left(U^{\prime}\right)=\operatorname{det}\left(A_{l_{1}, \cdots, l_{j-1}, i, l_{j+1}, \cdots, l_{r}}^{\prime}\right) \\
& =a_{i, j}^{\prime}
\end{aligned}
$$


Por lo tanto $A=A^{\prime}$, lo cual implica que $U=U^{\prime}$, concluyendo que $p$ es inyectiva.

Solo resta probar que la Grassmanniana $G(r, n)$ es un cerrado en $\mathbb{P}^{N-1}$ para concluir que $p$ es una inmersión cerrada. Este resultado se concluye a partir del siguiente teorema.

Teorema 1.15 (Relaciones de Plücker). La Grassmanniana $\mathrm{G}(\mathrm{r}, \mathrm{n}) \subset \mathbb{P}^{\mathrm{N}-1}$ es el conjunto de ceros de la siguiente relación de polinomios cuadráticos:

$$
\sum_{\lambda=1}^{r+1}(-1)^{\lambda} p_{i_{1}, \cdots, i_{r-1}, j_{\lambda}} p_{j_{1}, \cdots, \widehat{j_{\lambda}}, \cdots, j_{r+1}}
$$

donde $i_{1}, \cdots, i_{r-1} y j_{1}, \cdots, j_{r+1}$ son cualesquiera números entre 1 y $\mathrm{n}$.

Demostración. Sean $1 \leqslant i_{1}, \cdots, i_{r-1}, j_{1}, \cdots, j_{r+1} \leqslant n$ fijos. Sea $u \in$ $\mathrm{G}(\mathrm{r}, \mathrm{n})$ representado por la matriz $A$ de tamaño $n \times r$. Entonces la relación de Plücker evaluada en $\mathrm{U}$ es igual a

$$
\sum_{\lambda=1}^{r+1}(-1)^{\lambda} \operatorname{det}\left(A_{i_{1}, \cdots, i_{r-1}, j_{\lambda}}\right) \operatorname{det}\left(A_{j_{1}, \cdots, \widehat{j_{\lambda}}, \cdots, j_{r+1}}\right) .
$$

Expandiendo el primer determinante a lo largo de su última fila, tenemos:

$$
\sum_{\lambda=1}^{r+1}(-1)^{\lambda} \sum_{\mu=1}^{r}(-1)^{r+\mu} a_{j_{\lambda}, \mu} \operatorname{det}\left(A_{i_{1}, \cdots, i_{r-1}}^{\widehat{\mu}}\right) \operatorname{det}\left(A_{j_{1}, \cdots, \widehat{j_{\lambda}}, \cdots, j_{r+1}}\right),
$$

donde $A_{i_{1}, \cdots, i_{r-1}}^{\widehat{\mu}}$ es obtenida de $A_{i_{1}, \cdots, i_{r-1}}$ al eliminar la $\mu$-ésima columna. Ahora, esta última expresión puede ser escrita de la siguiente manera:

$$
\sum_{\mu=1}^{r}(-1)^{r+\mu} \operatorname{det}\left(A_{i_{1}, \cdots, i_{r-1}}^{\widehat{\mu}}\right) \sum_{\lambda=1}^{r+1}(-1)^{\lambda} a_{j_{\lambda}, \mu} \operatorname{det}\left(A_{j_{1}, \cdots, \widehat{j_{\lambda}}, \cdots, j_{r+1}}\right),
$$

pero esto es igual a

$$
\sum_{\mu=1}^{r}(-1)^{r+\mu} \operatorname{det}\left(A_{i_{1}, \cdots, i_{r-1}}^{\widehat{\mu}}\right)(-1) \operatorname{det}\left({ }^{\mu} A_{j_{1}, \cdots, \widehat{j_{\lambda}}, \cdots, j_{r+1}}\right),
$$

donde ${ }^{\mu} A_{j_{1}, \cdots, \widehat{j_{\lambda}}, \cdots, j_{r+1}}$ es obtenido de $A_{j_{1}, \cdots, \widehat{j_{\lambda}}, \cdots, j_{r+1}}$ al añadirle la $\mu$-ésima columna de $A$ como su primera su primera columna. Ahora, al variar $\mu$, de $1 \mathrm{a} r$, la matriz ${ }^{\mu} A_{j_{1}, \cdots, \widehat{j_{\lambda}}, \cdots, j_{r+1}}$ tiene dos columnas idénticas, entonces

$$
\operatorname{det}\left({ }^{\mu} A_{j_{1}, \cdots, \widehat{j_{\lambda}}, \cdots, j_{r+1}}\right)=0 .
$$

Por lo tanto la última expresión es igual a cero. De aquí que todo punto de $G(r, n)$ es cero de la ecuación.

Demostremos que cada punto $q=\left(q_{\underline{i}}\right)_{\underline{i} \in I_{r, n}}$ que satisface las relaciones de Plücker (RP) proviene de un elemento de $G(\bar{r}, n)$. Para ello sea $q=\left(q_{\underline{i}}\right)_{\underline{i} \in I_{r, n}}$ un cero de las RP, entonces existe $\underline{l} \in \mathrm{I}_{r, n}$ tal que $\mathrm{q}_{\underline{l}} \neq 0$, de hecho podemos tomar 
$\mathrm{q}_{\mathrm{l}}=1$. Primero demostremos que todas las entradas $\mathrm{q}_{\mathrm{j}}$ de $\mathrm{q}$ quedan determinadas por $r \cdot(n-r+1)+1$ de sus entradas, en donde $r \cdot(n-r+1)$ de dichas entradas $q_{\underline{i}}$ serán aquellas en las que $\underline{i}$ difiera de $\underline{l}$ por un entero en sus secuencias, es decir, $\#\{\underline{i} \cap \underline{l}\}=r-1$.

Sea $q_{j}$ una entrada de $q$ tal que hay $m$ elementos de $j$ que no están en $\underline{l}$, es decir, $\#\{\underline{j} \cap \underline{l}\}=r-m$. Consideremos la siguiente RP con las secuencias

$$
\left(j_{1}, \cdots, \hat{j_{\beta}}, \cdots, j_{r-1}, j_{r}\right) \quad y \quad\left(l_{1}, \cdots, l_{r}, j_{\beta}\right)
$$

siendo $j_{\beta}$ una de los elementos de $\underline{j}$ que no está en $\underline{l}$, entonces

$$
0=\sum_{\lambda=1}^{r+1}(-1)^{\lambda} \mathrm{q}_{j_{1}, \cdots, \widehat{j_{\beta}}, \cdots, j_{r-1}, j_{r}, l_{\lambda}} q_{l_{1}, \cdots, \widehat{l_{\lambda}}, \cdots, l_{r}, j_{\beta}}
$$

considerando el último elemento de la suma tenemos

$$
\mathrm{q}_{\underline{j}} \mathrm{q}_{\underline{\mathrm{l}}}=\sum_{\lambda=1}^{\mathrm{r}}(-1)^{\lambda} \mathrm{q}_{j_{1}, \cdots, \widehat{j_{\beta}}, \cdots, j_{r-1}, j_{r}, l_{\lambda}} q_{l_{1}, \cdots, \widehat{l_{\lambda}}, \cdots, l_{r}, j_{\beta}} .
$$

Observe que si $\boldsymbol{l}_{\lambda}$ está en $\{\underline{j} \cap \underline{l}\}$ entonces $q_{\left(j_{1}, \cdots, \widehat{j_{\beta}}, \cdots, j_{r-1}, j_{r}, l_{\lambda}\right)}=0$. Por otro lado si $l_{\lambda}$ no está entre los $j_{1}, \cdots, j_{r}$ hay exactamante $(m-1)$ elementos que difieren en ambas secuencias, es decir, si $\underline{j}_{\lambda, \beta}=q_{\left(j_{1}, \cdots, \widehat{j_{\beta}}, \cdots, j_{r-1}, j_{r}, l_{\lambda}\right)}$, entonces $r-(m-1)=\#\left\{\underline{j}_{\lambda, \beta} \cap \underline{l}\right\}$. Por lo tanto, en el lado derecho de la última igualdad, solamente aparecen los sumandos en los cuales $l_{\lambda}$ no está en $j$. Este proceso se puede realizar para cada sumando con la secuencia adecuada, es decir, para cada $j_{\alpha}$ uno de los $(m-1)$ elementos que no están en $\underline{l}$, consideremos las siguientes secuencias

$$
\left(j_{1}, \cdots, \widehat{j_{\alpha}}, \widehat{j_{\beta}}, \cdots, j_{r-1}, j_{r}, l_{\lambda}\right) \quad y \quad\left(l_{1}, \cdots, \widehat{l_{\lambda}}, \cdots, l_{r}, j_{\beta}, j_{\alpha}\right)
$$

entonces

$\mathrm{q}_{j_{1}, \cdots, \widehat{j_{\beta}}, \cdots, j_{r}, l_{\lambda}} q_{l_{1}, \cdots, \widehat{l_{\lambda}}, \cdots, l_{r}, j_{\beta}}=\sum_{s=1}^{r}(-1)^{s} q_{j_{1}, \cdots, \widehat{j_{\alpha}}, \widehat{j_{\beta}}, \cdots, j_{r}, l_{\lambda}, l_{s}} q_{l_{1}, \cdots, \widehat{l_{\lambda}}, \widehat{l_{s}} \cdots, l_{r}, j_{\beta}, j_{\alpha}}$.

Note que en esta nueva igualdad, el lado derecho hay exactamente $(m-2)$ elementos de $\left(j_{1}, \cdots, \widehat{j_{\alpha}}, \widehat{j_{\beta}}, \cdots, j_{r}, l_{\lambda}, l_{s}\right)$ que no están entre los de $\underline{l}$. Así que cada elemento de la suma inicial se puede expresar en términos de $\left(i_{1}, \cdots, i_{r}\right)$ en $I_{r, n}$ tales que $r-(m-2)=\#\{\underline{i} \cap \underline{l}\}$. Continuando con este proceso, tenemos

$$
\mathrm{q}_{\underline{j}} \mathrm{q}_{\underline{\underline{l}}}=\sum_{\underline{i}} \mathrm{c}_{\underline{i}} \mathrm{q}_{i_{1}, \cdots, i_{r}}
$$

tales que $\#\{\underline{i} \cap \underline{l}\}=r-1$, es decir difieren solamente en un elemento de la secuencia, denotemos por $\left(I_{r, n}\right)_{\underline{l}}$ a dicho conjunto de entradas. Se tienen un total de $(n-r+1) r$ de estas secuencias $\underline{i}$, un cálculo sencillo nos da dicho resultado, hay $(n-r+1)$ elementos que no están en $\underline{l}$ y $r$ posiciones en dónde poner cada uno de ellos, es decir, $(n-r+1) r$. Demostrando así que las entradas de q quedan determinadas por $(n-r+1) r+1$ de ellas (hay que añadirle la secuencia $l$ ). 
La razón de este resultado, es que con estas entradas $q_{\underline{i}}$ podemos construir una matriz de tamaño $n \times r$ de rango $r$. Definimos

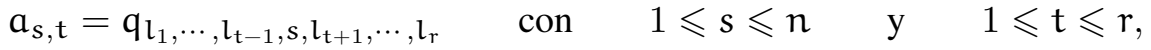

sea $A=\left(a_{s, t}\right)$. Por construcción $A_{l}=I d$, ya que si $t \in\left\{l_{r}, \cdots, l_{r}\right\}$ se tiene que $a_{s, t}=1$ si $s=t$ y $a_{s, t}=0$ si $s \neq t$, en particular, estos vectores columnas son linealmente independientes. También se tiene que $\operatorname{det} A_{\underline{l}}=1 \mathrm{y}$ por lo tanto el rango de $A$ es $r$, definamos por $U$ al subespacio vectorial representado por $A$. Ahora bien, sea $\underline{i} \in I_{r, n}$ tal que $\#\{\underline{i} \cap \underline{l}\}=r-1$ entonces existe un único $i_{s}$ que no pertenece a $\left\{l_{1}, \cdots, l_{r}\right\}$, por lo que $A_{\underline{i}}$ difiere de $A_{\underline{l}}$ en solamente una fila, la $i_{s}$ fila, luego, la entrada

$$
a_{i_{s}, i_{s}}=q_{l_{1}, \cdots, l_{i_{s}-1}, i_{s}, l_{i_{s}+1}, \cdots, l_{r}}=q_{\underline{i}} \quad \text { con } \quad q_{\underline{i}} \in\left(I_{r, n}\right)_{\underline{l}}
$$

Por otro lado, cada entrada de $A$ es obtenida por la regla de Cramer, tal como que se hizo en la demostración de la inyectividad de la aplicación de Plücker, por lo que tenemos que

$$
a_{i_{s}, i_{s}}=\operatorname{det} A_{l_{1}, \cdots, l_{i_{s}-1}, i_{s}, l_{i_{s}+1}, \cdots, l_{r}}=p_{l_{1}, \cdots, l_{i_{s}-1}, i_{s}, l_{i_{s}+1}, \cdots, l_{r}}(U)
$$

por lo tanto toda $q_{\underline{i}} \in\left(I_{r, n}\right)_{\underline{l}}$ es una coordenada de Plücker. De hecho la regla de Cramer demuestra que cada entrada de la matriz $A$ es una coordenada de Plücker que pertecene a $\left(I_{r, n}\right)_{\underline{l}}$ por lo que el determinante de cada menor de $A$ está en función de dichas coordenadas. Demostrando con esto que para toda entrada $q_{j}$ de $q$ satisfaciendo las RP es una coordenada de Plücker, es decir $q=p(U)$. Por lo tanto $G(r, n)$ es un cerrado en $\mathbb{P}^{N-1}$.

Este resultado se puede generalizar y para ello introduzcamos un poco de notación. Sea $S\left(a_{i}, \cdots, a_{t}\right)$ el grupo simétrico de $n$ letras.

Corolario 1.16 (Relaciones de Plücker generalizadas (RPG)). Para $\mathrm{s} \leqslant \mathrm{r}$ consideremos los siguientes grupos simétricos:

$$
\begin{aligned}
S & =S\left(i_{s}, \cdots, i_{r}, j_{1}, \cdots, j_{s}\right) \\
S^{\prime} & =S\left(i_{s}, \cdots, i_{r}\right) \times S\left(j_{1}, \cdots, j_{s}\right) \subset S .
\end{aligned}
$$

\section{Entonces}

$$
\sum_{\sigma \in S / S^{\prime}}(-1)^{\operatorname{sgn} \sigma} p_{i_{1}, \cdots, i_{s-1}, \sigma\left(i_{s}\right), \cdots, \sigma\left(i_{r}\right)} \cdot p_{\sigma\left(j_{1}\right), \cdots, \sigma\left(j_{s}\right), j_{s+1}, \cdots, j_{r}}=0
$$

Demostración. Sea $\tau \in S / S^{\prime}$ entonces $\tau=\sigma \sigma^{\prime}$ con $\sigma^{\prime} \in S^{\prime}$. Observemos que para toda $i_{\alpha}$ se tiene que $\sigma^{\prime}\left(i_{\alpha}\right) \in\left\{i_{s}, \cdots i_{r}\right\}$, a su vez, para toda $j_{\beta}$ se cumple que $\sigma^{\prime}\left(j_{\beta}\right) \in\left\{j_{1}, \cdots, j_{s}\right\}$. Lo cual implica que

$$
\begin{aligned}
& {\left[i_{1} \cdots i_{s-1} \sigma^{\prime}\left(i_{s}\right) \cdots \sigma^{\prime}\left(i_{r}\right)\right]=(-1)^{l}\left[i_{1} \cdots i_{r}\right],} \\
& {\left[\sigma^{\prime}\left(j_{1}\right) \cdots \sigma^{\prime}\left(j_{s}\right) j_{s+1} \cdots j_{r}\right]=(-1)^{\mathrm{t}}\left[j_{1} \cdots j_{r}\right] .}
\end{aligned}
$$

Por lo tanto $\tau$ solamente depende de $\sigma$.

Ahora bien, podemos ver la suma $(*)$ como una $r+1$ forma alternante en $E$ con 
$\operatorname{dim} E=r$. Para ello escribimos la matriz de coordenadas

$$
A=\left(\begin{array}{ccc}
x_{11} & \cdots & x_{1 r} \\
\vdots & & \vdots \\
x_{n 1} & \cdots & x_{n r}
\end{array}\right)=\left(\begin{array}{c}
\overline{X_{1}} \\
\vdots \\
\overline{X_{n}}
\end{array}\right) .
$$

Donde cada $\overline{X_{\gamma}}$ es una fila de $A$.

Observemos que la suma $(*)$ consiste en elegir, $\overline{X_{i_{s}}}, \cdots \overline{X_{i_{r}}}$ y $\overline{X_{j_{1}}}, \cdots \overline{X_{j_{s}}}$. Es decir $r+1$ vectores filas. Luego la suma $(*)$ es una forma alternante dado que es la suma y producto de funciones alternantes, esto es debido a que dicha suma está definida mediante determinantes. Por lo tanto hemos definido la $r+1$ forma alternante que deseábamos en un espacio de $\operatorname{dim} E=r$ por lo tanto la suma $\left(^{*}\right)$ es cero. Demostrando así el corolario.

\subsection{Variedades de Schubert}

Sea $E$ un espacio vectorial de dimensión $n$ sobre $k$. Sean $E_{t}$ los subespacios vectoriales de $E$ de dimensión $t$ generados por $\left\{e_{1}, \cdots, e_{t}\right\}$, los primeros $t$ elementos de la base canónica. Consideremos la siguiente bandera en $\mathrm{E}$ :

$$
\mathrm{E}_{0}=0 \subset \mathrm{E}_{1} \subset \mathrm{E}_{2} \subset \cdots \subset \mathrm{E}_{\mathrm{n}-1} \subset \mathrm{E}_{\mathrm{n}}=\mathrm{E} .
$$

Para cada $\underline{i} \in \mathrm{I}_{\mathrm{r}, \mathrm{n}}$ se define la variedad de Schubert asociada a $\underline{i}$ como

$$
X_{\underline{i}}=\left\{W \in G(r, n) \mid \operatorname{dim}_{k}\left(W \cap E_{i_{t}}\right) \geqslant t, \quad 1 \leqslant t \leqslant r\right\} .
$$

Proposición 1.17. $\mathrm{X}_{\underline{\mathrm{j}}} \subseteq \mathrm{X}_{\underline{i}}$ si y sólo si $\underline{\mathrm{j}} \leqslant \underline{\mathrm{i}}$.

Demostración. Supongamos que $\mathfrak{j} \leqslant \underline{i}$, entonces para toda $t$ se tiene que $j_{t} \leqslant i_{t}$ por lo que $E_{j_{t}} \subseteq E_{i_{t}}$, entonces, para todo $W \in X_{\underline{j}}$ se cumple $\left(W \cap E_{j_{t}}\right) \subseteq$ $\left(W \cap E_{i_{t}}\right)$, lo cual implica que $t \leqslant \operatorname{dim}\left(W \cap E_{j_{t}}\right) \leqslant \operatorname{dim}\left(W \cap E_{i_{t}}\right)$. Por lo tanto $W$ pertenece a $X_{i}$.

Para la otra implicación, procedamos por contradicción. Es decir, supongamos que $X_{j} \subseteq X_{\underline{i}}$ pero $\underline{j} \not \underline{i}$, es decir, existe $t$ tal que $i_{t}<j_{t}$ y $j_{l} \leqslant i_{l}$ para $1 \leqslant l \leqslant$ $t-1$. Para facilitar el análisis podemos suponer que $t=r$. Entonces, $E_{j_{l}} \subseteq E_{i_{l}}$ para $1 \leqslant l \leqslant r-1$ e $E_{i_{r}} \subset E_{j_{r}}$, por lo que $\left(W \cap E_{j_{l}}\right) \subseteq\left(W \cap E_{i_{l}}\right)$ lo cual implica que $l \leqslant \operatorname{dim}\left(W \cap E_{j_{l}}\right) \leqslant \operatorname{dim}\left(W \cap E_{i_{l}}\right)$. A su vez $\left(W \cap E_{i_{r}}\right) \subset\left(W \cap E_{j_{r}}\right)$ implica $r \leqslant \operatorname{dim}\left(W \cap E_{i_{r}}\right)<\operatorname{dim}\left(W \cap E_{j_{r}}\right)$. Ahora bien, podemos elegir $r$ vectores linealmente independientes que generen a $W$, de la siguiente manera: $\left\{v_{1}\right\} \subset(W \cap$ $\left.\mathrm{E}_{\mathrm{j}_{1}}\right) \subseteq\left(\mathrm{W} \cap \mathrm{E}_{\mathrm{i}_{1}}\right),\left\{v_{1}, v_{2}\right\} \subset\left(\mathrm{W} \cap \mathrm{E}_{\mathrm{j}_{2}}\right) \subseteq\left(\mathrm{W} \cap \mathrm{E}_{\mathrm{j}_{2}}\right)$, así sucesivamente hasta $\left\{v_{1}, \cdots, v_{r-1}\right\} \subset\left(W \cap E_{j_{r-1}}\right) \subseteq\left(W \cap E_{i_{r-1}}\right)$. Por último $u_{i_{r}} \in\left(W \cap E_{i_{r}}\right) \subset$ $\left(W \cap E_{j_{r}}\right)$ tal que $\left\{v_{1}, \cdots, v_{r-1}, u_{i_{r}}\right\}$ sean linealmente independientes, es decir, que generen a $W$. Luego escribamos cada vector en términos de los elementos de las bases de cada $E_{j_{t}}$, es decir:

$$
v_{t}=e_{j_{t}}+\sum_{l<j_{t}} a_{l, t} e_{j_{l}} \quad \text { y } \quad u_{i_{r}}=e_{j_{r}}+\sum_{l<j_{r}} a_{l, r} e_{j_{l}} .
$$

Sea $A^{\prime}=\left(a_{p, q}^{\prime}\right)$ la matriz asociada a esta descripción, en donde $a_{p, q}^{\prime}=0$ si $p>j_{q}$. Observe que $A_{\underline{j}}^{\prime}$ es una matriz triangular superior y unipotente, por lo 
tanto tiene inversa, entonces $A^{\prime} \cdot A_{\underline{j}}^{\prime-1}$ generan el mismo subespacio $W$ por lo tanto podemos suponer que $A^{\prime}$ es tal que $A_{j}^{\prime}=$ Id

Realicemos este mismo proceso para los elementos de las bases de cada $E_{i_{t}}$, entonces

$$
v_{t}=e_{i_{t}}+\sum_{l<i_{t}} a_{l, t} e_{i_{l}} \quad \text { y } \quad u_{i_{r}}=e_{i_{r}}+\sum_{l<i_{r}} a_{l, r} e_{i_{l}}
$$

y obtendremos una matriz $A=\left(a_{p, q}\right)$ tal que $A_{\underline{i}}=\operatorname{Id}$ y $a_{p, q}=0$ si $p>i_{q}$. Ambas matrices generan el mismo subespacio $W$ por lo que bajo la aplicación de Plücker deben de representar el mismo punto en $\mathbb{P}^{N-1}$. Sin embargo $p_{j}\left(A^{\prime}\right)=$ $\operatorname{det}\left(A_{\mathfrak{j}}^{\prime}\right)=1$ y $p_{\underline{i}}\left(A^{\prime}\right)=\operatorname{det}\left(A_{\underline{i}}^{\prime}\right)=0$ mientras que $p_{\underline{j}}(A)=\operatorname{det}\left(A_{\underline{j}}\right)=0$ y $p_{\underline{i}}(A)=\operatorname{det}\left(A_{\underline{i}}\right)=1$. Por lo que $p(A)$ y $p\left(A^{\prime}\right)$ no pertenecen al mismo punto en $\mathbb{P}^{\bar{N}-1}$, contradiciendo el hecho de que $A$ y $A^{\prime}$ generan el mismo espacio. Dicha contradicción proviene de suponer que $\mathfrak{j} \not \underline{i}$. Por lo tanto si $X_{j} \subseteq X_{\underline{i}}$ entonces $\underline{j} \leqslant \underline{i}$.

De la proposición anterior se obtienen los siguientes corolarios.

Corolario 1.18. $e_{\underline{j}} \in X_{\underline{i}}$ si y sólo si $\underline{j} \leqslant \underline{i}$.

Demostración. $e_{j} \in X_{j}$ puesto que estos subespacios satisfacen las condiciones de Schubert para la secuencia $\underline{j}$, dado que $X_{\underline{j}} \subseteq X_{\underline{i}}$ si y sólo si $\underline{j} \leqslant \underline{i}$ se cumple el corolario.

Corolario 1.19. Sean $\underline{\mathfrak{j}}, \underline{i}$ en $\mathrm{I}_{\mathrm{r}, \mathrm{n}}$ entonces

$$
p_{\underline{j}} \mid x_{\underline{i}} \neq 0 \Longleftrightarrow \underline{j} \leqslant \underline{i} \text {. }
$$

Demostración. Por el corolario anterior $e_{\underline{j}} \in X_{\underline{i}}$ si y sólo si $\underline{j} \leqslant \underline{i}$, luego $p_{\underline{j}}\left(e_{\underline{l}}\right) \neq 0$ si y sólo si $\underline{j}=\underline{l}$. Por lo tanto $p$ no se anula en $X_{\underline{i}}$ si y sólo si $\underline{j} \leqslant \underline{i}$.

\subsection{Teoría de monomios estándar en variedades de Schu- bert}

Sea $I(G(r, n))=\left\{f \in k\left[\wedge^{r} E\right]:\left.f\right|_{G(r, n)} \equiv 0\right\}$ el ideal homogéneo de anulación de la Grassmanniana y denotaremos por $R=k[G(r, n)]=k\left[\wedge^{r} E\right] / I(G(r, n))$ su anillo de coordenadas homogéneo.

Como el anillo de coordenadas homogéneo del espacio proyectivo $\mathbb{P}\left(\wedge^{r} E\right)$ es el anillo de polinomios $k\left[\wedge^{r} E\right]=k\left[p_{\underline{i}} \mid \underline{i} \in I_{r, n}\right]$, este anillo tiene una base de monomios en las coordenadas de Plücker. Nos interesa encontrar una base, a partir de estas coordenadas, de $\mathrm{k}[\mathrm{G}(\mathrm{r}, \mathrm{n})]$. Para ello haremos uso de la teoría de monomios estándar y su aplicación a las variedades de Schubert. Esto se debe a que para $\underline{i}=[n-r+1 \cdots n]$ se tiene que $X_{\underline{i}}=G(r, n)$, la teoría se desarrollará para cualquier variedad de Schubert en $G(r, n)$.

Sea $R=k[G(r, n)]$ el anillo de coordenadas homogéneo de $G(r, n)$. Cambiando la notación, las entradas de Plücker las denotaremos por $p_{\underline{i}}=\left[i_{1} \cdots i_{r}\right]$, el 
determinante del menor correspondiente. Entonces, dado un monomio $f$ en $R$

$$
f=\left[i_{11} \cdots i_{1 r}\right] \cdot\left[i_{21} \cdots i_{2 r}\right] \cdots\left[i_{h 1} \cdots i_{h r}\right]
$$

una forma conveniente de escribirlo es en forma matricial, es decir

$$
\left[\begin{array}{ccc}
i_{11} & \cdots & i_{i r} \\
\vdots & & \vdots \\
i_{h 1} & \cdots & i_{h r}
\end{array}\right]
$$

Definición 1.20. El monomio $f$ se dice que es estándar si en las filas es estrictamente creciente, es decir

$$
i_{\mathrm{t} 1}<i_{\mathrm{t} 2}<\cdots<i_{\mathrm{tr}} \quad \text { para cualquier } \mathrm{t}
$$

y en las columnas es creciente

$$
i_{1 s} \leqslant i_{2 s} \leqslant \cdots \leqslant i_{h s} \text { para cualquier s. }
$$

Note que podemos definir un orden total en las coordenadas de Plücker mediante un orden total en $I_{r, n}$.

Sean $\underline{i}, \underline{j}$ en $I_{r, n}$ entonces $\underline{i} \succ \underline{j}$ si y sólo si, existe $1 \leqslant t \leqslant r$ tal que

$$
i_{1}=j_{1}, \cdots i_{t-1}=j_{t-1}, i_{t}>j_{t} \text {. }
$$

Entonces dos coordenadas de Plücker, $p_{\underline{i}}, p_{\underline{j}}$,

$$
p_{\underline{i}} \succ p_{\underline{j}} \Leftrightarrow \underline{i} \succ \underline{j} .
$$

Esto nos permite definir un orden lexicográfico en el conjunto de monomios estándar, es decir, dados dos monomios estándar, $f, g$

$$
f=\left[\begin{array}{ccc}
i_{11} & \cdots & i_{1 r} \\
\vdots & & \vdots \\
i_{h 1} & \cdots & i_{h r}
\end{array}\right]<\left[\begin{array}{ccc}
j_{11} & \cdots & j_{1 r} \\
\vdots & & \vdots \\
j_{h 1} & \cdots & j_{h r}
\end{array}\right]=g
$$

si existe $t, 1 \leqslant t \leqslant h$ tal que $\left[i_{s 1} \cdots i_{s r}\right]=\left[j_{s 1} \cdots j_{s r}\right]$ si $s<t,\left[i_{t 1} \cdots i_{t r}\right]<$ $\left[j_{\mathrm{t} 1} \cdots j_{\mathrm{tr}}\right]$.

Proposición 1.21. Los monomios estándar generan a $\mathrm{R}$ como espacio vectorial.

Demostración. Sea $f$ un monomio no estándar. Basta considerar el caso cuando $f$ consta de dos variables,

$$
f=\left[\begin{array}{lll}
i_{1} & \cdots & i_{r} \\
j_{1} & \cdots & j_{r}
\end{array}\right]
$$

Que $f$ no sea estándar significa que existe $s$ tal que $i_{t} \leqslant j_{t}$ para $1 \leqslant t \leqslant s-1$ y $j_{s}<i_{s}$. Usando las relaciones de Plücker generalizadas, con la nueva notación tenemos

$$
\begin{aligned}
\sum_{\sigma \in S / S^{\prime}}(-1)^{\operatorname{sgn} \sigma_{f_{\sigma}}} & =\sum_{\sigma \in S / S^{\prime}}(-1)^{\operatorname{sgn} \sigma}\left[\begin{array}{ccccccc}
i_{1} & \cdots & i_{s-1} & \sigma\left(i_{s}\right) & \cdots & \sigma\left(i_{r}\right) & \\
& \sigma\left(j_{1}\right) & \cdots & \sigma\left(j_{s}\right) & j_{s+1} & \cdots & j_{r}
\end{array}\right] \\
& =0 .
\end{aligned}
$$


Para $\sigma=\mathrm{Id}, \mathrm{f}_{\sigma}=\mathrm{f}$ y de la ecuación anterior despejamos $\mathrm{f}$ y tenemos

$$
f=\sum_{I d \neq \sigma \in S / S^{\prime}}(-1)^{\operatorname{sgn} \sigma}\left[\begin{array}{ccccccc}
i_{1} & \cdots & i_{s-1} & \sigma\left(i_{s}\right) & \cdots & \sigma\left(i_{r}\right) & \\
& \sigma\left(j_{1}\right) & \cdots & \sigma\left(j_{s}\right) & j_{s+1} & \cdots & j_{r}
\end{array}\right] .
$$

Luego, para toda $\sigma \neq$ Id existe $l$ en $\left\{i_{s}, \cdots, i_{r}\right\}$ tal que $\sigma\left(i_{l}\right)=j_{t}, 1 \leqslant t \leqslant s$. Entonces para todo $s+1 \leqslant u \leqslant r$ y para todo $s \leqslant l \leqslant r$ se cumple que $j_{\mathfrak{u}} \geqslant i_{l}$ y para todo $1 \leqslant t \leqslant s$ se tiene $i_{\mathfrak{u}}>j_{t}$, lo cual implica $j_{u} \geqslant \sigma\left(i_{l}\right)$ para toda $s \leqslant l \leqslant r$. A su vez, $\sigma\left(j_{\alpha}\right) \geqslant i_{\beta}$ con $1 \leqslant \alpha \leqslant s$ y $1 \leqslant \beta \leqslant s-1$.

Tenemos dos casos para cada $\sigma \neq$ Id

(a). Caso 1: $f_{\sigma}$ con $\sigma\left(i_{s}\right)=i_{s}$, entonces existe $l \neq s, s+1 \leqslant l r$ tal que $\sigma\left(\mathfrak{i}_{l}\right)=j_{\mathrm{t}}$. Luego, reordenando la secuencia superior $\underline{i}$ se tiene una secuencia creciente:

$\left\{i_{1}<\cdots<i_{s-1}<\sigma\left(i_{\lambda}\right)<\cdots<\sigma\left(i_{l}\right)=j_{t}<\cdots<\sigma\left(i_{s}\right)=i_{s}<\cdots<\sigma\left(i_{\delta}\right)\right.$, dado que $\sigma\left(i_{s}\right)$ ocupa $u$-ésimo sitio, $u>s$ tenemos que $j_{u} \geqslant \sigma\left(i_{s}\right)=i_{s}$ y $\sigma\left(j_{\alpha}\right) \geqslant i_{\beta}$ con $1 \leqslant \alpha \leqslant s$ y $1 \leqslant \beta \leqslant s-1$. Por lo tanto $f_{\sigma}$ es estándar.

(b). Caso $2: f_{\sigma}$ con $\sigma\left(i_{s}\right)=j_{l^{\prime}}$, sabemos que $j_{u} \geqslant \sigma\left(i_{l}\right)$ para toda $s \leqslant l \leqslant r$ y $s+1 \leqslant u \leqslant r$ por lo tanto $j_{\mathfrak{u}} \geqslant \sigma\left(i_{l^{\prime}}\right)$. Luego, al reordenar la secuencia se obtiene que $f_{\sigma}$ es un monomio estándar.

Por lo tanto $f$ se puede expresar como combinación de $f_{\sigma}$ siendo éstos, monomios estándar, es decir, generan a R.

Ahora aplicaremos la teoría de monomios estándar a las variedades de Schubert. Sea $R=k\left[G_{r, n}\right]$, para $\underline{i}$ en $I_{r, n}$ sea $R(\underline{i})=k\left[X_{\underline{i}}\right]$ el anillo de coordenadas de la variedad de Schubert $X_{\underline{i}}$. Dado un monomio estándar $f_{\tau}, \tau=\underline{i}_{1} \cdots \underline{i}_{h}$

$$
f_{\tau}=p_{\tau}=\left[\begin{array}{ccc}
i_{11} & \cdots & i_{1 r} \\
\vdots & & \vdots \\
i_{h 1} & \cdots & i_{h r}
\end{array}\right]
$$

definimos que $f$ es estándar en $X_{\underline{i}}$ si $\underline{i} \geqslant \underline{i}_{h}$, lo cual es equivalente a decir que $f$ no se anula en todo $X_{i}$. Por lo tanto un monomio no estándar $f,\left.f\right|_{X_{i}}$ es cero o su residuo es estándar en $X_{\underline{i}}$. Entonces, dado que los monomios estándar generan a $R$, basta considerar la restricción de éstos a la variedad de Schubert $X_{\underline{i}}$. Por el análisis anterior, los monomios estándar que al restringirlos a $X_{\underline{i}}$ no son estándar, se anulan en toda la variedad por lo que forman una base para el ideal de anulación de $X_{\underline{i}}$, luego, los monomios estándar tales que su restricción a $X_{\underline{i}}$ sigue siendo estándar en $X_{\underline{i}}$ generan $R_{\underline{i}}$. Sólo resta demostrar que son una base para $R_{\underline{i}}$ es decir, la siguiente proposición.

Proposición 1.22. Los monomios estándar en $\mathrm{X}_{i}$ son linealmente independientes.

Demostración. Procederemos por inducción sobre la dimensión de $X_{i}$. Si $\operatorname{dim}\left(X_{\underline{i}}\right)=0$ entonces $\underline{i}=[12 \cdots r]$ o bien $p_{\underline{i}}=p_{\text {Id }}$ por lo tanto $X_{\underline{i}}=\left\{E_{r}\right\}$ es decir es un punto, por lo que $k\left[X_{\underline{i}}\right]=k\left[p_{I d}\right]$ es decir solamente un monomio estándar y se cumple la hipótesis. 
Si $\operatorname{dim}\left(X_{\underline{i}}\right)>0$, y supongamos que se cumple para $\operatorname{dim}\left(X_{\underline{i}}\right)=d-1$ y probemos para $d$, es decir se cumple para todas las subvariedades de $X_{\underline{i}}$ de dimensión menor a d. Para este paso aplicaremos inducción sobre el grado de los monomios estándar. Es decir, sea

$$
\sum_{l=1} c_{\tau_{l}} f_{\tau_{l}}=0
$$

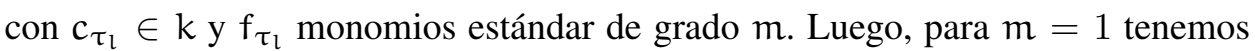
las coordenadas de Plücker, es decir, $f_{\tau_{l}}=p_{j_{1}}$ para $\underline{i} \geqslant j_{l} \in I_{r, n}$, las cuales son linealmente independientes, ya que si existiese $c_{\tau_{1^{\prime}}} \neq 0$ entonces, $c_{\tau_{1^{\prime}}} p_{j_{1^{\prime}}} \neq 0$ por lo que $c_{\tau_{l^{\prime}}} p_{\underline{j}_{\underline{j}^{\prime}}}\left(e_{\underline{j}_{l^{\prime}}}\right) \neq 0$ y $c_{\tau_{l}} p_{\underline{j}_{l}}\left(e_{\underline{j}_{\underline{j}^{\prime}}}\right)=0$ implicando que la suma no se anula en $e_{j_{1}}$ lo cual es una contradicción. Por lo tanto $c_{\tau_{l}}=0$ para toda $l$.

Sea $m>1$, supongamos que se cumple para monomios estándar en $X_{\underline{i}}$ de grado $m-1$ y probemos para $m$. Entonces podemos asumir que existe $f_{\tau_{l}}$ tal que $\tau_{l}=\left\{\underline{j}_{l_{1}}, \underline{j}_{l_{2}}, \cdots, \underline{j}_{l_{h}}\right\}$ y $\underline{j}_{l_{h}} \neq \underline{i}$ (ya que $\underline{i}$ no se anula en $X_{\underline{i}}$ ). Ahora bien, consideremos la restricción de la suma a $X_{\underline{j}_{l_{h}}}$, entonces los monomios estándar, $f_{\tau_{s}}$, que no son estándar en $X_{\underline{j}_{l_{h}}}$ se anulan completamente, por otro lado existe al menos un monomio estándar que sigue siendo estándar en $X_{\underline{j}_{h_{h}}}$ a saber, $f_{\tau_{l}}$, por lo tanto la suma es una relación de monomios estándar en $X_{\underline{j}_{h_{h}}}$ no vacía la cual no se anula completamente en $X_{\underline{j}_{l_{h}}}$. Por la hipótesis de inducción, esto es una contradicción.

Por lo tanto la suma

$$
\sum_{l=1} c_{\tau_{l}} f_{\tau_{l}}=0
$$

si y sólo si $\mathrm{c}_{\tau_{l}}=0$ para toda $l$.

De estas dos últimas proposiciones se tiene.

Corolario 1.23. Los monomios estándar en $\mathrm{X}_{\underline{i}}$ son una base para $\mathrm{R}_{\underline{\mathrm{i}}}$.

Hemos dado una base explícita para el ideal de anulación de cualquier variedad de Schubert y para su anillo de coordenadas, sin embargo aún falta verificar que este ideal es radical para poder tener el siguiente morfismo de k-álgebras:

$$
\pi: \mathrm{R} \longrightarrow \mathrm{R}(\underline{\mathfrak{i}}) \Longrightarrow \mathrm{R}(\underline{\mathfrak{i}})=\mathrm{R} / \operatorname{ker}(\pi)
$$

donde $\pi$ es el morfismo de restricción y denotemos por $\mathrm{J}_{\underline{i}}=\operatorname{ker}(\pi)$ y por $\mathrm{I}_{\underline{i}}$ el ideal de anulación de $X_{\underline{i}}$.

Proposición 1.24. El ideal $\mathrm{I}_{\underline{i}}=\mathrm{J}_{\underline{i}}$, es decir, $\mathrm{I}_{\underline{i}}$ es radical.

Demostración. Es claro que se tiene una inclusión directa, $\mathrm{I}_{\underline{i}} \subset \mathrm{J}_{\underline{i}}$. Para la otra inclusión consideremos $f \in R$ entonces podemos escribirlo como una combinación de monomios estándar en $R$

$$
f=\sum a_{\tau_{l}} f_{\tau_{l}}+\sum b_{\gamma_{t}} h_{\gamma_{t}}
$$

en donde cada monomio de la primera suma, $f_{\tau_{l}} \operatorname{con} \tau_{l}=\left\{\tau_{l 1}, \cdots \tau_{l s}\right\}$, satisface que $\underline{i} \not \tau_{\mathrm{ls}}$ y cada monomio de la segunda suma, $h_{\gamma_{\mathrm{t}}}$ con $\gamma_{\mathrm{t}}=\left\{\gamma_{\mathrm{t} 1}, \cdots \gamma_{\mathrm{ts}}\right\}$ 
satisface que $\underline{i} \geqslant \gamma_{\mathrm{ts}}$. Dado que $\sum a_{\tau_{l}} f_{\tau_{l}} \in I_{\underline{i}}$ se tiene que $f \in J_{\underline{i}}$

$$
\begin{aligned}
& \Longleftrightarrow \sum \mathrm{b}_{\gamma_{\mathrm{t}}} \mathrm{h}_{\gamma_{\mathrm{t}}} \in \mathrm{J}_{\underline{\mathrm{i}}} \text { ya que } \quad \mathrm{I}_{\underline{\mathrm{i}}} \subset \mathrm{J}_{\underline{\mathrm{i}}} \\
& \Longleftrightarrow \pi(f)=0 \\
& \Longleftrightarrow \sum \mathrm{b}_{\gamma_{\mathrm{t}}} \mathrm{h}_{\gamma_{\mathrm{t}}}=0 \text { en } \mathrm{X}_{\underline{\mathrm{i}}} \\
& \Longleftrightarrow \mathrm{b}_{\gamma_{\mathrm{t}}}=0 \text { para toda } \mathrm{t} \text { (por la independencia lineal de los monomios estándar en } X_{\underline{i}} \text { ) } \\
& \Longleftrightarrow f=\sum a_{\tau_{l}} f_{\tau_{l}} \\
& \Longleftrightarrow f \in I_{i} \text {. }
\end{aligned}
$$

Por lo tanto $\mathrm{J}_{\underline{i}}=\mathrm{I}_{\underline{i}}$, es decir, es un ideal radical. 



\section{Capítulo 2}

\section{Acciones de grupos algebraicos}

En este capítulo se dará la definición de una acción algebraica de un grupo algebraico afín $\mathrm{G}$ sobre una variedad algebraica $X$, propiedades de dicha definición y una aplicación que nos permitirá caracterizar todos los grupos algebraicos afines, finalizando el capítulo con los teoremas fundamentales de la teoría de invariantes clásica.

Sea $G$ un grupo algebraico afín y $X$ una variedad algebraica. Se dice que $\alpha$ : $\mathrm{G} \times \mathrm{X} \longrightarrow \mathrm{X}$ es una acción regular izquierda si es un morfismo entre variedades algebraicas y $\alpha$ es una acción abstracta ( la acción de un grupo en un conjunto), es decir si se cumple:

(a). Para todo elemento $x$ de $X$ se tiene:

$$
\alpha(g h, x)=\alpha(g, \alpha(h, x))
$$

para cualesquiera elementos $g, h$ de $G$.

(b). Para todo elemento $x$ de $X$ se tiene que

$$
\alpha(e, x)=x .
$$

Por conveniencia denotaremos por $\alpha(\mathrm{g}, \mathrm{\chi})=\mathrm{g} \chi \mathrm{y}$ a partir de ahora, por acción de $G$ en $X$, nos referiremos a una acción regular izquierda, con la salvedad que se especifique lo contrario. En la literatura, también se dice que $X$ es una $G$-variedad.

De manera análoga se define una acción regular derecha, $\alpha: X \times G \longrightarrow X$.

Ejemplo 2.1. Existen aplicaciones naturales provenientes de una acción de $\mathrm{G}$ en $\mathrm{X}$ :

(a). Sea $g \in G$ definimos una traslación izquierda, $\lambda_{g}$, por

$$
\begin{gathered}
\lambda_{g}: X \longrightarrow X \\
x \longmapsto g x
\end{gathered}
$$

y por traslación derecha $\rho_{g}$, por

$$
\begin{gathered}
\rho_{g}: X \longrightarrow X \\
x \longmapsto x g .
\end{gathered}
$$

Ambas traslaciones son isomorfismos en $X$.

(b). Sea $x \in X$, definimos la aplicación órbita, $\pi_{x}$ por

$$
\begin{gathered}
\pi_{x}: G \longrightarrow X \\
g \longmapsto g x .
\end{gathered}
$$


Observación 2.2. Recordemos que dado un morfismo entre variedades afines, se tiene un homomorfismo de k-álgebras, en particular para una acción $\alpha$ de $\mathrm{G}$ en $\mathrm{X}$. Es decir:

$$
\alpha: G \times X \rightarrow X
$$

induce

$$
\alpha^{*}: k[X] \rightarrow k[G] \otimes_{k} k[X]
$$

por lo tanto para todo elemento $f$ en $k[X]$ tenemos que $\alpha^{*}(f)=\sum_{i=1}^{n} h_{i} \otimes_{k} f_{i}$.

De esta observación se tiene que, mediante las traslaciones, $\lambda_{g}$ y $\rho_{g}$, de la variedad $X$, podemos definir nuevas traslaciones en el anillo de funciones de $X$, $k[X]$.

Sea $g \in G$. Para toda función $f \in k[X]$ definimos

(a). $L_{g}: k[X] \rightarrow k[X]$ por $L_{g}(f)(x)=\left(\lambda_{g} f\right)(x)=f \circ \lambda_{g^{-1}}(x)=f\left(g^{-1} x\right)$

(b). $R_{g}: k[X] \rightarrow k[X]$ por $R_{g}(f)(x)=\left(\rho_{g} f\right)(x)=f \circ \rho_{g}(x)=f(x g)$

Estas traslaciones, $L_{g}$ y $R_{g}$ nos permiten inducir una acción de $G$ en $k[X]$, a la izquierda:

$$
\begin{gathered}
\alpha_{*}: G \times k[X] \rightarrow k[X] \\
\alpha_{*}(g, f)=L_{g}(f)=f \circ \lambda_{g^{-1}} .
\end{gathered}
$$

Siempre que se tiene una acción $\mathrm{G} \times \mathrm{X} \longrightarrow \mathrm{X}$ se tienen los conjuntos siguientes:

Definición 2.3. Sea $G$ un grupo algebraico y $X$ una $G$-variedad:

(a). Si $x \in X$, la órbita de $x$ es $\pi_{x}(G):=\{g \cdot x \mid g \in G\} \subset X$ y lo denotaremos por órb ${ }_{G}(x)$ o bien $G \cdot x$.

(b). El estabilizador o subgrupo de isotropía de $\chi \in X$ es $\mathrm{G}_{x}:=\{\mathrm{g} \in \mathrm{G} \mid \mathrm{g} \cdot \chi=$ $x\} \subseteq \mathrm{G}$. Si $\mathrm{G}_{\chi}=\mathrm{G}$ se dice que $x$ es un punto fijo bajo la acción de $\mathrm{G}$. Denotaremos $X^{G}$ al conjunto de todos los puntos fijos.

(c). Diremos que $X$ es un espacio homogéneo si la acción de $G$ en $X$ es transitiva, es decir, si existe $\chi \in X$ tal que órb ${ }_{\mathrm{G}}(x)=X$, es decir, sólo hay una órbita.

También podemos definir funciones entre G-variedades.

Definición 2.4. Sea $G$ un grupo algebraico afín y sean $X, Y$ dos $G$-variedades. Un morfismo de variedades algebraicas $\mu: X \rightarrow Y$ es llamado un G-morfismo o un morfismo equivariante o morfismo de G-variedades si $\mu(a \cdot x)=a \mu(x)$ para toda $a \in G$ y para toda $x \in X$, es decir si el siguiente diagrama conmuta

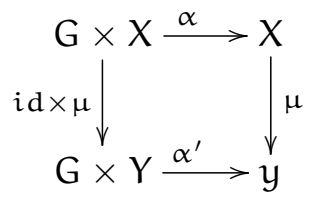

Para poder demostrar que el subgrupo de isotropía de una acción es un subgrupo cerrado en $\mathrm{G}$ daremos la siguiente definición. 
Definición 2.5. Sea $G$ un grupo algebraico afín y $X$ una $G$-variedad. Si $Y, Z \subset X$ son subconjuntos, definimos el transportador de $\mathrm{Y}$ a $\mathrm{Z}$ como:

$$
\operatorname{Tran}_{G}(Y, Z)=\{a \in G \mid a \cdot Y \subset Z\}
$$

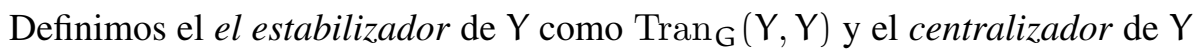
como $\mathfrak{C}(\mathrm{Y})=\bigcap_{\mathrm{y} \in \mathrm{Y}} \mathrm{G}_{\mathrm{y}}$

Definición 2.6. Un conjunto $W \subset X$ se dice que es G-estable o G-invariante si $\mathrm{G} \cdot \mathrm{W} \subset \mathrm{W}$. Un ejemplo de conjuntos G-estables son las órbitas de la acción.

Con esto ya podemos enunciar el siguiente teorema.

Teorema 2.7. Sea $\mathrm{G}$ un grupo algebraico afin y $\mathrm{X}$ una $\mathrm{G}$-variedad.

(a). Si $\mathrm{Y}, \mathrm{Z} \subset \mathrm{X}$ subconjuntos tal que $\mathrm{Z}$ es cerrado, entonces $\operatorname{Tran}_{\mathrm{G}}(\mathrm{Y}, \mathrm{Z}) \subset$ $\mathrm{G}$ es cerrado

(b). El subgrupo de isotropía de $\mathrm{x} \in \mathrm{X}$ es un subgrupo cerrado. En particular $\mathfrak{C}_{\mathrm{G}}(\mathrm{Y})$ es un subrupo cerrado.

(c). El conjunto de $\mathrm{X}^{\mathrm{G}}$ de puntos fijos de $\mathrm{X}$ es cerrado.

(d). Si G es conexo, entonces $\mathrm{G}$ estabiliza todas las componentes irreducibles de X.

Demostración. (a). Sea $\pi_{x}: G \rightarrow X$ la aplicación órbita. Si $y \in Y$ se tiene que $\pi_{y}^{-1}(Z)=\{a \in G \mid \quad a \cdot y \in Z\}$. Dado que $\pi_{y}$ es una función continua y $Z$ es cerrado, $\pi_{y}^{-1}(Z)$ es cerrado en $G$. Entonces

$$
\bigcap_{y \in Y} \pi_{y}^{-1}(Z)=\{g \in G \mid \quad g \cdot y \in Z \quad \forall y \in Y\}=\operatorname{Tran}_{G}(Y, Z) .
$$

Por lo tanto es cerrado en $\mathrm{G}$.

(b). Por el inciso anterior

$$
\operatorname{Tran}_{\mathrm{G}}(\{x\},\{x\})=\{\mathrm{g} \in \mathrm{G} \mid \mathrm{g} \cdot \mathrm{x}=\mathrm{x}\}=\mathrm{G}_{\mathrm{x}}
$$

es cerrado. A su vez $\mathfrak{C}_{\mathrm{G}}(Y)$ es cerrado al ser intersección de cerrados.

(c). Consideremos $\varphi_{\mathrm{a}}: \mathrm{X} \rightarrow \mathrm{X}$ definida como $\varphi_{\mathrm{a}}(\mathrm{x})=\mathrm{a} \cdot x$ con $\mathrm{a} \in \mathrm{G}$. Ahora bien, sabemos que la gráfica

$$
\Gamma\left(\varphi_{a}\right)=\{(x, y) \in X \times X \mid \quad y=a x\}
$$

es cerrada en $X \times X$ (ver prop. 2.12 de [12] ). Luego la imagen inversa de la aplicación diagonal

$$
\Delta^{-1}\left(\Gamma\left(\varphi_{a}\right)\right)=\left\{x \in X \mid \quad x=\varphi_{a}(x)=a x\right\}
$$

es el conjunto de puntos fijos por $a, X^{a}$ es cerrado en $X$. Entonces

$$
\bigcap_{\mathrm{a} \in \mathrm{G}} \mathrm{X}^{\mathrm{a}}=\{x \in X \mid \quad x=\mathrm{a} x \forall \mathrm{a} \in \mathrm{G}\}=\mathrm{X}^{\mathrm{G}}
$$

es cerrado en $X$. 
(d). Supongamos que $G$ es conexo, por lo tanto irreducible. Sea $X_{1} \subset X$ una componente irreducible de $X$. Consideremos el morfismo $\varphi$ proveniente de la acción de $\mathrm{G}$ en $\mathrm{X}$. Entonces $\mathrm{G} \cdot \mathrm{X}_{1}=\varphi\left(\mathrm{G} \times \mathrm{X}_{1}\right)$ entonces $\mathrm{G} \cdot \mathrm{X}_{1}$ es irreducible, ya que es la imagen del producto de irreducibles. Por otro lado $X_{1}=e \cdot X_{1} \subset G \cdot X_{1}$ implicando que $X_{1}=G \cdot X_{1}$ por la condición de irreducibilidad de $X_{1}$.

Observación 2.8. Sea $x \in X$. Consideremos $Y=\overline{\operatorname{orb}_{\mathrm{G}}(x)}$, la cerradura de la órbita. $\mathrm{Y}$ es un conjunto $\mathrm{G}$-estable, esto se concluirá a partir del siguiente teorema.

Teorema 2.9. Sea $\mathrm{G}$ un grupo algebraico afin y $\mathrm{X}$ una $\mathrm{G}$-variedad, entonces para todo $\mathrm{x} \in \mathrm{X}$ la órbita órb $\mathrm{o}_{\mathrm{G}}(\mathrm{x})$ es abierto en órb $\mathrm{o}_{\mathrm{G}}(\mathrm{x})$.

Demostración. Sea $x \in X$, consideremos el morfismo dominante $\varphi_{x}$ : $\mathrm{G} \rightarrow \mathrm{Y}=\overline{\text { orb }_{\mathrm{G}}(x)}$. Entonces por Chevalley existe $\mathcal{U} \subset$ órb $_{\mathrm{G}}(x)$ tal que es abierto en $Y$ y $\bar{U}=Y$. Dado que $G$ actúa transitivamente en órb $b_{G}(x)$ se tiene que $\bigcup_{g \in G} g \cdot \mathcal{U}=\operatorname{órb}_{\mathrm{G}}(x)$ por lo tanto la órbita de $x$ es un abierto en $Y$.

Ahora bien, para demostrar la G-estabilidad de $Y=\overline{\operatorname{orb}_{\mathrm{G}}(\mathrm{X})}$ denotemos por $\mathcal{U}=$ órb $_{\mathrm{G}}(\mathrm{x})$, entonces por el teorema anterior $\mathcal{U}$ es abierto y denso en $\mathrm{Y}$, entonces:

$$
\begin{aligned}
\mathrm{G} \cdot \mathrm{Y} & =\alpha(\mathrm{G} \times \mathrm{Y})=\alpha(\mathrm{G} \times \overline{\mathcal{U}})=\alpha(\overline{\mathrm{G}} \times \overline{\mathcal{U}}) \\
& \subseteq \alpha(\overline{\mathrm{G} \times \mathcal{U}})=\subseteq \overline{\alpha(\mathrm{G} \times \mathcal{U})}=\overline{\mathcal{U}}=\mathrm{Y}
\end{aligned}
$$

Una propiedad importante de los grupos algebraicos afines es que son isomorfos a un subgrupo cerrado de algún $\mathrm{GL}_{n}$. Para demostrar esto requerimos del siguiente teorema:

Teorema 2.10. Sea $\mathrm{F}$ un subespacio de dimensión finita de $\mathrm{k}[\mathrm{X}]$. Entonces existe un subespacio $\mathrm{E}$ de $\mathrm{k}[\mathrm{X}]$ de dimensión finita tal que

(a). $\mathrm{F} \subseteq \mathrm{E}$ estable bajo traslaciones izquierdas de $\mathrm{G}$.

(b). Una condición necesaria y suficiente para que F sea invariante bajo traslaciones izquierdas es que

$$
\alpha^{*} \mathrm{~F} \subset \mathrm{k}[\mathrm{G}] \otimes_{\mathrm{k}} \mathrm{F} .
$$

Demostración. Basta demostrar el caso cuando $F$ es de dimensión 1, es decir $F=\langle t f\rangle$ con $f$ en $k[X]$. Recordando la observación 2.2 tenemos que:

$$
\alpha^{*}(f)=\sum_{i=1}^{n} h_{i} \otimes_{k} f_{i}
$$

con $f_{i}$ en $k[G]$ y $h_{i}$ en $k[X]$, con $n$ mínimo. Por otro lado, para toda $g \in G$ se tiene que $\left(\lambda_{g} f\right)(x)=f\left(g^{-1} x\right)$ es decir:

$$
f\left(g^{-1} x\right)=(f \circ \alpha)\left(g^{-1}, x\right)=\alpha^{*}(f)(x)=\sum_{i=1}^{n} h_{i}\left(g^{-1}\right) \cdot f_{i}(x)
$$

por lo tanto $\left(\lambda_{g} f\right)=\sum_{i=1}^{n} h_{i}\left(g^{-1}\right) \cdot f_{i}$ es decir, existe un subespacio de dimensión finita que contiene a $\lambda_{g} f($ para toda $g \in G)$, el generado por $W=\left\langle f_{i}\right\rangle$. Al 
considerar $E=\left\langle\lambda_{g} f\right\rangle_{g \in G}$ este es un subespacio invariante bajo las traslaciones y de dimensión finita puesto que está contenido en $W$.

Para el inciso restante, consideremos lo siguiente: si $\left\{f_{i}\right\}$ es una base para $F$, existen $h_{j}$ en $k[X]$ tales que $\left\{f_{i}\right\} \cup\left\{h_{j}\right\}$ es una base de $k[X]$ y entonces para cualquier $f \in k[X]$

a su vez

$$
f=\sum_{i} f_{i}+\sum_{j} h_{j}
$$

$$
\alpha^{*} f=\sum_{i} r_{i} \otimes_{k} f_{i}+\sum_{j} s_{j} \otimes_{k} h_{j}
$$

donde $r_{i}, s_{j}$ perteneces a $k[G]$.

Si $f \in F$ y $g \in G$ tenemos que

$$
\lambda_{g} f=\sum_{i} r_{i}\left(g^{-1}\right) \cdot f_{i}+\sum_{j} s_{j}\left(g^{-1}\right) \cdot h_{j} .
$$

Entonces, $\lambda_{g} f$ está en $F$ si y sólo si $s_{j}\left(g^{-1}\right)=0$ para toda $j$. Al variar $g$ en $G$ y $f$ en $F$, tenemos que $\lambda_{g} F$ está contenido en $F$ si y sólo si $s_{j}\left(g^{-1}\right)=0$ para toda $j$, es decir,

$$
\lambda_{g} F \subset F \Longleftrightarrow \alpha^{*} F \subset k[G] \otimes_{k} F
$$

Si $X=G$, podemos definir una acción que es tanto izquierda como derecha mediante traslaciones:

$$
\begin{gathered}
\mu:(G \times G) \times G \rightarrow G \\
\mu((g, h) x)=g \times h^{-1} .
\end{gathered}
$$

A partir de esto podemos inducir dos acciones en el anillo de coordenadas de $k[\mathrm{G}]$ como se hizo en la observación 2.2. En este caso dichas traslaciones son homomorfismos de G.

Aplicando el teorema anterior tenemos.

Corolario 2.11. Cada subespacio de dimensión finita $\mathrm{F}$ de $\mathrm{k}[\mathrm{G}]$ está contenido en un subespacio de dimensión finita estable bajo traslaciones que son tanto izquierdas como derechas.

La importancia de la existencia de tal subespacio invariante es garantizar que las traslaciones pertenecen a algún espacio de matrices invertibles, con lo cual podemos demostrar el siguiente teorema.

Teorema 2.12. Sea $\mathrm{G}$ un grupo algebraico afín. Entonces $\mathrm{G}$ es isomorfo a un subgrupo cerrado de algún $\mathrm{GL}_{n}$.

Demostración. Sabemos que $k[G]=k\left[f_{1}, f_{2}, \cdots, f_{n}\right]$ y por el teorema y el corolario anterior podemos elegir a $f_{1}, f_{2}, \cdots, f_{n}$ como una base de un subespacio $E$ de $k[G]$ invariante bajo traslaciones izquierdas y derechas. Con ello podemos definir los automorfismos

$$
R_{\mathrm{g}}: k[\mathrm{G}] \rightarrow \mathrm{k}[\mathrm{G}]
$$

y por la invarianza de $E$, para toda $f$ en $k[G]$ se tiene que $R_{g} f$ pertenece a $G L(E)$. 
Continuando con la notación previa, tenemos:

$$
\mu: \mathrm{G} \times \mathrm{G} \rightarrow \mathrm{G} \Longrightarrow \mu^{*}: \mathrm{k}[\mathrm{G}] \rightarrow \mathrm{k}[\mathrm{G}] \otimes_{\mathrm{k}} \mathrm{k}[\mathrm{G}] .
$$

De nuevo, por el teorema anterior $\mu^{*} E \subset E \otimes_{k} k[G]$, entonces para cada $i$ se tiene

$$
\mu^{*} f_{i}=\sum_{i} f_{j} \otimes_{k} m_{i, j}
$$

para algún $m_{i, j}$ en $k[G]$.

En particular para cada $f_{i}$ elementos de la base de $E$

$$
\left(R_{g} f_{i}\right)(x)=\sum_{j=1}^{n} f_{j}(x) \cdot m_{i, j}(g) \Longrightarrow R_{g} f_{i}=\sum_{j=1}^{n} m_{i, j}(g) \cdot f_{j} .
$$

Con ello podemos definir el siguiente homomorfismo de grupos

$$
\alpha: \mathrm{G} \longrightarrow \mathrm{GL}(\mathrm{E}) \simeq \mathrm{GL}_{\mathrm{n}}
$$

dada por $\alpha(g)=\left(m_{i, j}(g)\right)$. A su vez esto nos define un homomorfismo de $k$ álgebras

$$
\alpha^{*}: k\left[\mathrm{GL}_{n}\right]=\mathrm{k}\left[\mathrm{T}_{1,1}, \mathrm{~T}_{1,2}, \cdots, \mathrm{T}_{\mathrm{n}, \mathrm{n}}, \mathrm{det}\right] \longrightarrow \mathrm{k}[\mathrm{G}]
$$

dado por $\alpha^{*}\left(T_{i, j}\right)=\left(m_{i, j}\right)$. Entonces para toda $i$

$$
f_{i}(x)=f_{i}(e x)=\sum_{j=1}^{n} f_{j}(e) \cdot m_{i, j}(x)
$$

dado que $f_{i}$ generan a $k[G]$ se tiene que $m_{i, j}$ también. Con lo cual $\alpha^{*}$ es epimorfismo implicando que $\alpha$ es inyectivo. Así $G$ es isomorfo a su imagen $\alpha(G)$ contenida en $\mathrm{GL}_{n}$.

\subsection{Teoremas fundamentales}

Denotemos por $M_{m \times n}=M=M(m, n)$ el conjunto de todas las matrices de tamaño $m \times n$ definidas sobre un campo $k$. $M$ es la variedad algebraica afín $\mathbb{A}^{m n}$ que tiene por anillo de coordenadas $R=k\left[\left(x_{i, j}\right)\right]$. Definamos por $M_{r}=$ $M_{r}(m, n)$ como las matrices de rango a lo más $r$. Usaremos la teoría de monomios estándar en variedades de Schubert para demostrar que el ideal de anulación de $M_{r}$, definámoslo por $\mathrm{I}_{\mathrm{r}+1}$, está dado por los $(\mathrm{r}+1) \times(\mathrm{r}+1)$ menores. La prueba recae en demostrar que $\mathrm{I}_{\mathrm{r}+1}$ es un ideal radical.

Primero cada matriz $\left(a_{i, j}\right)$ de $M$ le podemos asociar un elemento en la Grassmanniana $G(n, m+n)$, el cual está generado por la siguiente matriz

$$
\left(\begin{array}{ccc}
a_{1,1} & \cdots & a_{1, n} \\
\vdots & & \vdots \\
a_{m, 1} & \cdots & a_{m, n} \\
0 & \cdots & 1 \\
\vdots & & \vdots \\
1 & \cdots & 0
\end{array}\right) .
$$


Consideremos el siguiente menor $[m+1 \cdots m+n]$, es decir elijamos las últimas $n$ filas. Esto nos define un morfismo entre $M$ y el abierto donde no se anula $[m+1 \cdots m+n]$, de hecho es un isomorfismo. Sea $R^{\prime}=k[G(n, m+n)]$, por lo tanto

$$
R=R^{\prime} /([m+1 \cdots m+n] \pm 1) .
$$

Ahora bien, por los resultados de la sección 2 del capítulo 1, se sabe que una base para la variedad de Schubert $k\left[X_{[m+1 \cdots m+n]}\right]$ está dada por los monomios estándar en $R^{\prime}$ tales que son estándar en $X_{[m+1 \cdots m+n]}$, por lo tanto una base para $\mathrm{R}$ son los monomios estándar $\boldsymbol{f}_{\tau} \operatorname{con} \tau=\left\{\underline{\underline{i}}_{1}, \cdots, \underline{\mathfrak{i}}_{h}\right\}$ en $X_{[\mathrm{m}+1 \cdots \mathrm{m}+\mathrm{n}]}$, tales que $\underline{i}_{h} \neq[m+1 \cdots m+n]$.

Observación 2.13. Sea $1 \leqslant s \leqslant n$, consideremos un menor de tamaño $s \times s$ en $M$, demostraremos que dicho menor se puede extender a una coordenada de Plücker en $G(n, m+n)$. Consideremos un menor de tamaño $s \times s$ formado por las filas $i_{1}, \cdots, i_{s}$ y las columnas $j_{1}, \cdots, j_{s}$. Ahora bien, la columna $j_{l}$ tiene la entrada $a_{m+n+1-j_{l}, j_{l}}=1$ es decir, intersecta a la fila $i_{m+n+1-j_{l}}$ en la única entrada de la fila que es diferente de cero. Sea $Z=\left\{m+n+1-j_{s}, \cdots m+n+1-j_{1}\right\}$, el subconjunto de las filas $\{m+1, \cdots, m+n\}$, en donde cada columna $j_{l}$ intersecta a dichas filas en su única entrada diferente de cero. Entonces tomemos las $n-s$ filas faltantes para acompletar una coordenada de Plücker de $G(n, m+n)$ del complemento de Z. Entonces tenemos el determinante del menor formado por

$$
p_{\underline{i}}=\left[i_{1}, \cdots, i_{s}, i_{s+1}, \cdots, i_{n}\right]
$$

$\operatorname{con}\left\{i_{s+1}, \cdots, i_{n}\right\}$ en el complemento de $Z$. El cual al desarrollar este determinante a travez de sus últimas $n-s$ filas, $\left\{i_{s+1}, \cdots, i_{n}\right\}$, es decir, solamente sobreviven las columnas $\left\{j_{1}, \cdots, j_{s}\right\}$ por lo tanto $p_{\underline{i}}$ es igual al determinante del menor con el que iniciamos.

Por lo tanto todo menor de tamaño $s \times s$ se puede extender a una coordenada de Plüker y con ello podemos dar una base de monomios estándar en $R$ para $I_{s}$. Entonces una base de $\mathrm{I}_{\mathrm{S}}$ está dada por los monomios estándar

$$
\left[\begin{array}{ccc}
i_{11} & \cdots & i_{1 n} \\
\vdots & & \vdots \\
i_{t 1} & \cdots & i_{t n}
\end{array}\right],
$$

tales que $i_{1 s} \leqslant m y\left[i_{t 1} \cdots i_{t n}\right] \neq[m+1 \cdots m+n]$. Consecuentemente una base para el anillo cociente $D_{s}=k\left[\left(x_{i, j}\right)\right] / I_{s}$ consiste de todos los monomios estándar

$$
\left[\begin{array}{ccc}
i_{11} & \cdots & i_{1 n} \\
\vdots & & \vdots \\
i_{t 1} & \cdots & i_{t n}
\end{array}\right],
$$

tales que $i_{1 s}>m$ y $\left[i_{t 1} \cdots i_{t n}\right] \neq[m+1 \cdots m+n]$.

El siguiente teorema a demostrar el es primer teorema fundamental de la teoría de invariantes, para ello denotemos por $\mathrm{G}=\mathrm{GL}_{\mathrm{r}}(\mathrm{k})$ y consideremos la siguiente 
variedad $M(m, r) \times M(r, n)=M^{\prime}$ con $r \leqslant \min (m, n)$. Definamos la siguiente acción de $G$ en $M(m, r) \times M(r, n)$

$$
\begin{aligned}
\alpha: M(m, r) \times M(r, n) & \longrightarrow M(m, r) \times M(r, n) \\
\alpha(g,(A, B)) & =\left(A g^{-1}, g B\right)
\end{aligned}
$$

Dado que $\alpha$ está definido mediante la multiplicación de matrices, es decir, es polinomial, $\alpha$ es un morfismo regular. Por otro lado es claro que $\alpha$ es una acción, sean $\mathrm{g}, \mathrm{h} \in \mathrm{G}$ entonces

$$
\begin{aligned}
\alpha(h g,(A, B)) & =\left(A(h g)^{-1},(h g) B\right)=\left(A g^{-1} h^{-1}, h g B\right)=\left(\left(\mathrm{Ag}^{-1}\right) h^{-1}, h(g B)\right) \\
& =(h,(A g-1, g B))=(h, \alpha(g,(A, B)))
\end{aligned}
$$

Ahora bien, la multiplicación matricial $\mu: M(m, r) \times M(r, n) \longrightarrow M(m, n)$ tiene por imagen a $M_{r}(m, n)$. Observese que $\mu$ está bien definida en las órbitas de $\alpha$ ya que para toda $g \in G$ y para todo $\operatorname{par}(A, B) \in M(m, r) \times M(r, n)$ se tiene que $\mu\left(A g^{-1}, g B\right)=A g^{-1} g B=A B$, en realidad, permite extender la acción de $G$ a $M_{r}(m, n)$, sienda esta acción trivial, es decir, $g C=C$ y $\mu$ es invariante bajo la acción de G.

Luego para toda $A \in M(m, r)$ y $B \in M(r, n)$ los podemos escribir de la siguiente manera

$$
(A, B)=\left(\left(\begin{array}{l}
A^{\prime} \\
A^{\prime \prime}
\end{array}\right), \quad\left(\begin{array}{ll}
B^{\prime} & B^{\prime \prime}
\end{array}\right)\right), \text { donde } A^{\prime}, B^{\prime} \in M(r, r) .
$$

Con esta descripción podemos definir una función determinante $\mathrm{d}$

$$
\mathrm{d}(\mathrm{A}, \mathrm{B})=\operatorname{det}\left(\mathrm{A}^{\prime} \mathrm{B}^{\prime}\right)
$$

Dado que $\mu$ es un suprayectiva en su imagen, se tiene que para toda $C \in$ $M_{r}(m, n)$ existe un par $(A, B) \in M(m, r) \times M(r, n)$ tales que $C=\mu(A, B)=$ $A B$ entonces podemos definir $d$ en $M_{r}(m, n)$ mediante $d(C)=\operatorname{det}\left(A^{\prime} B^{\prime}\right)$ con $A^{\prime}, B^{\prime}$ como se definió previamente. Observe que $d$ no depende de la representación de $C$, supongamo que existen $A, B, E, F$ tales que $A B=C=E F$, entonces,

$$
\left.(A, B)=\left(\left(\begin{array}{l}
A^{\prime} \\
A^{\prime \prime}
\end{array}\right), \quad\left(\begin{array}{ll}
B^{\prime} & B^{\prime \prime}
\end{array}\right)\right) \quad y \quad(E, F)=\left(\begin{array}{c}
E^{\prime} \\
E^{\prime \prime}
\end{array}\right), \quad\left(\begin{array}{ll}
F^{\prime} & F^{\prime \prime}
\end{array}\right)\right),
$$

entonces

$$
(A B)=\left(\begin{array}{ll}
A^{\prime} B^{\prime} & A^{\prime} B^{\prime \prime} \\
A^{\prime \prime} B^{\prime} & A^{\prime \prime} B^{\prime \prime}
\end{array}\right)=C=\left(\begin{array}{cc}
E^{\prime} F^{\prime} & E^{\prime} F^{\prime \prime} \\
E^{\prime \prime} F^{\prime} & E^{\prime \prime} F^{\prime \prime}
\end{array}\right)=(E F)
$$

entonces $A^{\prime} B^{\prime}=E^{\prime} F^{\prime}$, por lo tanto $\operatorname{det}\left(A^{\prime} B^{\prime}\right)=\operatorname{det}\left(E^{\prime} F^{\prime}\right)$, es decir, $d$ no depende de la representación de $C$.

Obsérvese que d es invariante bajo la acción de $\mathrm{G}$, puesto que para toda $\mathrm{g} \in \mathrm{G}$ se tiene

$$
\mathrm{d}\left(\left(A g^{-1}, \mathrm{gB}\right)\right)=\mathrm{d}\left(\left(\begin{array}{c}
A^{\prime} g^{-1} \\
A^{\prime \prime} g^{-1}
\end{array}\right), \quad\left(\begin{array}{ll}
g B^{\prime} & \left.g B^{\prime \prime}\right)
\end{array}\right)=\operatorname{det}\left(A^{\prime} g^{-1} g B^{\prime}\right)=\operatorname{det}\left(A^{\prime} B^{\prime}\right)\right.
$$

Con este análisis ya podemos enunciar el siguiente lema. 
Lema 2.14. Sea $S=k[M(m, r) \times M(r, n)]$ el anillo de coordenadas $M(m, r) \times$ $M(r, n)$ y $D=k\left[M_{r}(m, n)\right]$ entonces

$$
\mathrm{D}[1 / \mathrm{d}]=\mathrm{S}[1 / \mathrm{d}]^{\mathrm{G}} .
$$

Demostración. Dado que $G$ actúa en $M(m, r) \times M(r, n)$ induce una acción a su anillo de coordenadas $S$, a su vez $G$ actúa trivialmente en $M_{r}(m, n)$ e induce una acción trivial en $\mathrm{D}$.

Demostremos la inclusión $D[1 / d] \subset S[1 / d]^{G}$. Sea $C \in M_{r}(m, n)$ con $C=$ $(A, B)$ entonces, $d(C) \neq 0$ si y sólo si $\operatorname{det}\left(A^{\prime} B^{\prime}\right) \neq 0$ si y sólo si $\operatorname{det}\left(A^{\prime}\right), \operatorname{det}\left(B^{\prime}\right) \neq$ 0 es decir, si y sólo si rango $\left(A^{\prime}\right)=r=\operatorname{rango}\left(B^{\prime}\right)$. Ahora bien, para toda $g \in G$ se tiene que $\mathrm{Ag}^{-1}, \mathrm{gB}$ preservan el rango, por lo tanto si $\mathrm{W}$ es el abierto donde no se anula $d$, se tiene que $W$ es $G$-invariante. Por otro lado tenemos:

$$
\mu: M(m, r) \times M(r, n) \rightarrow M_{r}(m, n) \Rightarrow \mu^{*}: D \rightarrow S \Rightarrow \mu^{*}: D[1 / d] \rightarrow S[1 / d]
$$

Dado que $\mu$ es suprayectiva, implica que $\mu^{*}$ es inyectiva. Sea $h \in D[1 / d]$ entonces $h \circ \mu \in S[1 / d]$, luego $((h \circ \mu) g)(A, B)=h\left(\mu\left(A g^{-1}, g B\right)\right)=h(A B)=h(C)=$ $(h \circ \mu)(A, B)$ es decir es G-invariante, por lo tanto $D[1 / d] \subseteq S[1 / d]^{G}$.

Para la inclusión faltante, de nuevo denotemos por $W$ el abierto en $M(m, r) \times$ $M(r, n)$ donde $d$ no se anula, es decir, $W=\{(A, B) \mid \operatorname{rango}(A)=r=\operatorname{rango}(B)\}$. Definamos $V=\left\{(A, B) \mid A^{\prime}=I d\right\} \subset W$. Definimos una acción de $G$ en $G \times V$ de la siguiente manera

$$
\begin{aligned}
\mathrm{G} \times(\mathrm{G} \times \mathrm{V}) & \longrightarrow \mathrm{G} \times \mathrm{V} \\
(\mathrm{g},(\mathrm{h}, \mathrm{v})) & \longmapsto(\mathrm{gh}, v) .
\end{aligned}
$$

Es claro que esto es una acción. Por otro lado definamos el siguiente morfismo

$$
\begin{aligned}
\varphi: G \times V & \longrightarrow W \\
(g, v)) & \longmapsto g v
\end{aligned}
$$

donde gv es la acción $\alpha$ de G en W.

De hecho $\varphi$ es un morfismo G-invariante, ya que

$$
\begin{aligned}
\varphi(h,(g, u)) & =\varphi(h g, u)=(h g) u=\left(A(h g)^{-1},(h g) B\right) \\
& =h\left(A g^{-1}, g B\right)=h \varphi(g, u) .
\end{aligned}
$$

Este morfismo es un isomorfismo, para demostrarlo definamos la inversa

$$
\psi:\left(\left(\begin{array}{c}
A^{\prime} \\
A^{\prime \prime}
\end{array}\right), \quad\left(\begin{array}{ll}
B^{\prime} & B^{\prime \prime}
\end{array}\right)\right) \longmapsto\left(\left(\begin{array}{c}
I d \\
A^{\prime \prime}\left(A^{\prime}\right)^{-1}
\end{array}\right), \quad\left(\begin{array}{ll}
A^{\prime} B^{\prime} & \left.A^{\prime} B^{\prime \prime}\right)
\end{array}\right)\right.
$$

Demostremos que $\varphi \circ \psi=\mathrm{Id}, \operatorname{sean}(\mathrm{A}, \mathrm{B}) \in \mathrm{W}$, entonces 


$$
\begin{aligned}
& (\varphi \circ \psi)(A, B)=\varphi\left(\psi\left(\left(\left(\begin{array}{l}
A^{\prime} \\
A^{\prime \prime}
\end{array}\right), \quad\left(\begin{array}{ll}
B^{\prime} & B^{\prime \prime}
\end{array}\right)\right)\right)\right) \\
& =\varphi\left(\left(A^{\prime}\right)^{-1},\left(\left(\begin{array}{c}
I d \\
A^{\prime \prime}\left(A^{\prime}\right)^{-1}
\end{array}\right), \quad\left(\begin{array}{ll}
A^{\prime} B^{\prime} & \left.A^{\prime} B^{\prime \prime}\right)
\end{array}\right)\right)\right. \\
& =\left(\begin{array}{c}
\operatorname{Id} \\
A^{\prime \prime}\left(A^{\prime}\right)^{-1}
\end{array}\right) A^{\prime}, \quad\left(A^{\prime}\right)^{-1}\left(\begin{array}{ll}
A^{\prime} B^{\prime} & \left.A^{\prime} B^{\prime \prime}\right)
\end{array}\right) \\
& =\left(\left(\begin{array}{c}
A^{\prime} \\
A^{\prime \prime}
\end{array}\right), \quad\left(\begin{array}{ll}
B^{\prime} & B^{\prime \prime}
\end{array}\right)\right) \\
& =(\mathrm{A}, \mathrm{B})
\end{aligned}
$$

Verifiquemos $\psi \circ \varphi=\mathrm{Id}$

$$
\begin{aligned}
& (\psi \circ \varphi)\left(g,\left(\left(\begin{array}{c}
\text { Id } \\
A^{\prime \prime}
\end{array}\right),\left(\begin{array}{ll}
B^{\prime} & B^{\prime \prime}
\end{array}\right)\right)=\psi\left(\left(\left(\begin{array}{c}
I d \\
A^{\prime \prime}
\end{array}\right) g^{-1}, g\left(\begin{array}{ll}
B^{\prime} & B^{\prime \prime}
\end{array}\right)\right)\right)\right. \\
& =\psi\left(\left(\left(\begin{array}{c}
g^{-1} \\
A^{\prime \prime} g^{-1}
\end{array}\right),\left(\begin{array}{ll}
g B^{\prime} & g B^{\prime \prime}
\end{array}\right)\right)\right) \\
& =\left(g,\left(\begin{array}{c}
\mathrm{Id} \\
A^{\prime \prime} g^{-1} \mathrm{~g}
\end{array}\right),\left(\begin{array}{ll}
g^{-1} \mathrm{gB}^{\prime} & \mathrm{g}^{-1} \mathrm{gB} \mathrm{B}^{\prime \prime}
\end{array}\right)\right. \\
& =\left(g,\left(\begin{array}{c}
I d \\
A^{\prime \prime}
\end{array}\right),\left(\begin{array}{ll}
B^{\prime} & B^{\prime \prime}
\end{array}\right)\right)
\end{aligned}
$$

Por lo tanto $\varphi$ es un isomorfismo G-invariante.

Por lo tanto $k[G \times V] \simeq k[W]$ entonces $k[G \times V]^{G} \simeq k[W]^{G}$. Sea $f \in K[W]$ entonces $f \circ \varphi \in k[G \times V]$. Entonces $(f \circ \varphi)(g, v)=f(g v)=(f g)(v)$. Por lo que si $f \in k[W]^{G}$ se tiene que para toda $g \in G,(f \circ \varphi)(g, v)=f(g v)=(f g)(v)=$ $\mathrm{f}(v) \in \mathrm{k}[\mathrm{V}]$ por lo tanto, $k[\mathrm{~W}]^{\mathrm{G}} \subseteq \mathrm{k}[\mathrm{V}]$.

Ahora bien, sea $v \in \mathrm{V}$, entonces

$$
v=\left(\left(\begin{array}{c}
\mathrm{Id} \\
\mathrm{A}^{\prime \prime}
\end{array}\right),\left(\begin{array}{ll}
\mathrm{B}^{\prime} & \mathrm{B}^{\prime \prime}
\end{array}\right)\right)
$$

Para toda $\mathrm{g} \in \mathrm{G}$ tenemos que existe $w \in W$ tal que

$$
v=g w=\left(\left(\begin{array}{c}
g \\
A^{\prime \prime} g^{-1}
\end{array}\right) g^{-1}, g\left(g^{-1} B^{\prime} g^{-1} B^{\prime \prime}\right)\right) .
$$

Si $w, w^{\prime} \in W$ son tales que $v=g w=g^{\prime} w^{\prime}$ se tiene que $w$ y $w^{\prime}$ pertenecen a la misma órbita y dado que $W$ es $G$-inviarante, órb $b_{\mathrm{G}}(w) \in W$. Por lo que podemos concluir que $v$ parametriza la órbita de $w$, entonces si $f \in k[V]$ se tiene

$$
f(v)=f\left(o_{r b}(w)\right)
$$

es decir $f \in k[W]^{\mathrm{G}}$, por lo tanto $k[\mathrm{~V}] \subseteq k[\mathrm{~W}]^{\mathrm{G}}$, demostrando que $k[\mathrm{~V}]=k[\mathrm{~W}]^{\mathrm{G}}$. De esto igualdad podemos observar que si $f \in k[W]^{G}=S[1 / d]^{G}$ y

$$
w=\left(\left(\begin{array}{c}
A^{\prime} \\
A^{\prime \prime}
\end{array}\right),\left(\begin{array}{ll}
B^{\prime} & B^{\prime \prime}
\end{array}\right)\right) \text {. }
$$


en realidad está definida en

$$
v=\left(\left(\begin{array}{c}
\operatorname{Id} \\
A^{\prime \prime}\left(A^{\prime}\right)^{-1}
\end{array}\right),\left(\begin{array}{ll}
A^{\prime} B^{\prime} & A^{\prime} B^{\prime \prime}
\end{array}\right)\right) .
$$

es decir en $A^{\prime \prime}\left(A^{\prime}\right)^{-1}, A^{\prime} B^{\prime}$ y $A^{\prime} B^{\prime \prime}$. Por otro lado, sea $\mu(v)=\mu(w)=C$, por álgebra lineal sabemos que

$$
\left(A^{\prime}\right)^{-1}=\frac{1}{\operatorname{det}\left(A^{\prime}\right)} \operatorname{adj}\left(A^{\prime}\right) \quad \text { y } \quad\left(B^{\prime}\right)^{-1}=\frac{1}{\operatorname{det}\left(B^{\prime}\right)} \operatorname{adj}\left(B^{\prime}\right)
$$

entonces

$$
\begin{aligned}
A^{\prime \prime}\left(A^{\prime}\right)^{-1} & =A^{\prime \prime}\left(A^{\prime}\right)^{-1}\left(B^{\prime}\right)^{-1} B^{\prime} \\
& =A^{\prime \prime}\left(\frac{1}{\operatorname{det}\left(A^{\prime}\right)} \operatorname{adj}\left(A^{\prime}\right)\right)\left(\frac{1}{\operatorname{det}\left(B^{\prime}\right)} \operatorname{adj}\left(B^{\prime}\right)\right) B^{\prime} \\
& =\frac{1}{d(C)}\left(\operatorname{adj}\left(A^{\prime}\right) \operatorname{adj}\left(B^{\prime}\right)\right) A^{\prime \prime} B^{\prime} .
\end{aligned}
$$

Es decir, $f$ también está definida en

$$
\frac{1}{d} A^{\prime \prime} B^{\prime}
$$

Luego

$$
\mu(v)=C=\left(\begin{array}{ll}
A^{\prime} B^{\prime} & A^{\prime} B^{\prime \prime} \\
A^{\prime \prime} B^{\prime} & A^{\prime} B^{\prime \prime}
\end{array}\right) .
$$

Entonces si $f \in S[1 / d]^{G}$ está definida en cada una de las entradas de $C, f \in D$ pero la condición

$$
\frac{1}{d} A^{\prime \prime} B^{\prime}
$$

implica que $f \in D[1 / d]$. Quedando así demostrado el lema.

Con esto ya podemos enunciar el primer teorema fundamental.

Teorema 2.15 (Primer teorema fundamental). El anillo D es el anillo de invariantes de $\mathrm{S}$ bajo la acción de $\mathrm{G}$,

$$
\mathrm{S}^{\mathrm{G}}=\mathrm{D}
$$

Demostración. Por el lema anterior tenemos que

$$
\mathrm{D} \longrightarrow \mathrm{D}[1 / \mathrm{d}]=\mathrm{S}[1 / \mathrm{d}]^{\mathrm{G}}
$$

Dado que $d$ es $G$-invariante, se tiene que si $f \in D[1 / d]=S[1 / d]^{G}$ entonces $f$ es G-invariante por lo tanto $f \in S^{G}$ por lo tanto $D \subseteq S^{G}$.

Para la otra inclusión, si $f \in S^{G} \longrightarrow S[1 / d]^{G}=D[1 / d]$ entonces $f \in D[1 / d]$ por lo tanto existe $p$ tal que $f d^{p} \in D$. Por lo que basta probar que si $f \in S$ y $d f \in D$ se tiene que $f \in D$, ya que $S^{G} \subset S$.

Para comenzar, podemos identificar $M(m, n)$ como un abierto de $G(n, m+n)$, es 
decir, asociando a cada matriz $\left(a_{i, j}\right)$ con el subespacio vectorial generado por las columnas de

$$
\left(\begin{array}{ccc}
a_{1,1} & \cdots & a_{1, n} \\
\vdots & & \vdots \\
a_{m, 1} & \cdots & a_{m, n} \\
0 & \cdots & 1 \\
\vdots & & \vdots \\
1 & \cdots & 0
\end{array}\right)
$$

donde $d=[1 \cdots r, m+1 \cdots m+n-r]$ ya que la función d está determinada por el bloque de $r \times r$ de las primeras $r$-filas y $r$-columnas. Usando la base asociada de monomios estándar (descrita en la observación anterior), podemos escribir cada elemento de $f \in D$ de la siguiente manera

$$
\mathrm{df}=\sum_{i} \alpha_{i} T_{i}
$$

donde cada $T_{i}$ es un monomio estándar de la forma

$$
\left[\begin{array}{ccc}
i_{11} & \cdots & i_{1 n} \\
\vdots & & \vdots \\
i_{t 1} & \cdots & i_{t n}
\end{array}\right],
$$

tales que $i_{1, k+1}>m$ y $\left[i_{t} 1 \cdots i_{t} n\right] \neq[m+1 \cdots m+n]$. Consideremos los ceros de la hipersuperficie $d=0, Z$, entonces por la igualdad se tiene que $\sum_{i} \alpha_{i} T_{i}$ se anula en $Z$, por lo tanto existe un entero $p$ tal que

$$
\mathrm{d} \gamma=\left(\sum_{i} \alpha_{i} \mathrm{~T}_{i}\right)^{p}
$$

con $\gamma \in \mathrm{D}$, es decir $\sum_{i} \alpha_{i} \mathrm{~T}_{i}$ está en el radical de d. Luego dado que $\gamma \in \mathrm{D}$ podemos escribirlo en términos de monomios estándar

$$
\gamma=\sum_{j} \beta_{j} S_{j} .
$$

Sin pérdida de generalidad, podemos suponer que $T_{1}$ es el monomio máximo en el orden lexicográfico, $T_{1} \geqslant T_{i}$, entonces:

$$
\begin{aligned}
\left(\sum_{i} \alpha_{i} T_{i}\right)^{p} & =\alpha_{1}{ }^{p} T_{1}{ }^{p}+\sum_{i \neq 1}\left(\alpha_{i} T_{i}\right)^{l} \\
& =\sum_{j} \beta_{j} d S_{j}
\end{aligned}
$$

donde $\sum_{i \neq 1} \alpha_{i} T_{i}{ }^{l}$ es una suma monomios menores en el orden lexicográfico a $T_{1}$. Luego, para toda $j$ se tiene que $d S_{j}$ es un monomio estándar, ya que $d$ es el monomomio más grande en el orden lexicográfico en D. Ahora comparando ambas sumas término a término tenemos que el monomio mayor $T_{1}$ es igual al término mayor de la suma de los $d S_{j}$, denotémoslo por $d S_{j_{1}}$, por lo tanto $T_{1}=d S_{j_{1}}$ es 
decir, $\mathrm{T}_{1}$ es divisible por $\mathrm{d}$. Aplicando las relaciones de Plücker generalizadas, podemos escribir

$$
\sum_{i \neq 1}\left(\alpha_{i} T_{i}\right)^{l}
$$

como una combinación de monomios estándar, por lo tanto podemos repetir el proceso anterior y obtener que cada $T_{i}$ es divisible por $d$, por lo tanto

$$
f=\sum_{i} \alpha_{i}\left(T_{i} / d\right)
$$

es decir, $f$ pertenece a D. Demostrando que si $f \in S^{G}$ entonces está en D.

Hemos dado una base explícita para $\mathrm{I}_{s}$ y $\mathrm{D}_{s}$. Para ver que en efecto es el ideal de anulación de $M_{s}$ se necesita que $I_{s}$ sea un ideal radical.

Teorema 2.16 (Segundo teorema fundamental). El ideal de anulación de $\mathrm{M}_{\mathrm{r}}$ es el ideal generado por los menores $\mathrm{r}+1 \times \mathrm{r}+1$.

Demostración. Es claro que para todo elemento $A \in M_{r}$ se anula en el determinante de un menor de tamaño $r+1 \times r+1$ puesto que tiene rango menor igual a $r$. Por lo que resta ver que $I_{r+1}$ es radical. Consideremos la siguiente suma finita de monomios estándar $k\left[\left(x_{i, j}\right)\right]$

$$
\sum_{l} a_{l} F_{\tau_{l}}
$$

tales que $F_{\tau_{l}}$ no pertenece a $I_{r+1}$, es decir

$$
F_{\tau_{l}}=\left[\begin{array}{ccc}
i_{\tau_{l 11}} & \cdots & i_{\tau_{l 1 n}} \\
\vdots & & \vdots \\
i_{\tau_{l t 1}} & \cdots & i_{\tau_{l t n}}
\end{array}\right],
$$

en donde $i_{\tau_{l 1, r}}>m$. Sin pérdida de generalidad podemos suponer que $F_{\tau_{1}}$ es el monomio máximo bajo el orden lexicográfico. Supongamos que existe q tal que

$$
\left(\sum_{l} a_{l} F_{\tau_{l}}\right)^{q} \in I_{r+1}
$$

o bien

$$
\left(\sum_{l} a_{l} F_{\tau_{l}}\right)^{q}=a_{1} F_{\tau_{1}}^{q}+\sum_{l \neq 1}\left(a_{l} F_{\tau_{l}}\right)^{p}
$$

Observe que

$$
\sum_{l \neq 1}\left(a_{l} F_{\tau_{l}}\right)^{p}
$$

no son monomios estándar necesariamente, sin embargo por las relaciones de Plücker generalizadas, esta suma la podemos llevar, en un número finito de pasos, a 
una suma de monomios estándar estrictamente menores a $F_{\tau_{1}}^{q}$ en el orden lexicográfico. Por otro lado, existen monomios estándar $T_{\alpha} \in I_{r+1}$ con $i_{\alpha_{u 1, r}} \leqslant m$, únicos ya que son base de $\mathrm{I}_{\mathrm{r}+1}$, tales que

$$
a_{1} F_{\tau_{1}}^{q}+\sum_{l \neq 1}\left(a_{l} F_{\tau_{l}}\right)^{p}=\sum_{u, s} b_{\alpha_{u}} T_{\alpha_{u}} s
$$

Sea $T_{\alpha_{1}}$ (haciendo abuso de notación) el mayor de estos monomios estándar en el orden lexicográfico. Para que estas sumas sean iguales, es decir, estos dos polinomios sean iguales, es necesario que sean iguales término a término. Por lo que al comparar los términos máximos en ambas sumas, es decir, $\mathrm{F}_{\tau_{1}}^{\mathrm{q}}$ con $\mathrm{T}_{\alpha_{1}}{ }^{\mathrm{s}}$ deberían ser iguales, lo cual no es posible por las condiciones $i_{\tau_{11, r}}>\mathrm{me} i_{\alpha_{u 1, r}} \leqslant \mathrm{~m}$, obligando a que $a_{1}$ sea igual a 0 para poder preservar la igualdad. Realizando este proceso con

$$
\sum_{l \neq 1}\left(a_{l} F_{\tau_{l}}\right)^{p}
$$

se llega a que cada coeficiente debe de ser igual a cero. Por lo tanto si $\mathrm{F}^{\mathrm{q}} \in \mathrm{I}_{\mathrm{r}+1}$ entonces $\mathrm{F} \in \mathrm{I}_{\mathrm{r}+1}$, es decir, $\mathrm{I}_{\mathrm{r}+1}$ es radical.

\subsection{Cocientes}

La pregunta natural que surge cuando se tiene una G-variedad X es si el conjunto de órbitas

$$
\mathrm{X} / \mathrm{G}=\left\{\operatorname{orb}_{\mathrm{G}}(x) \mid x \in \mathrm{X}\right\}
$$

es de nuevo una variedad algebraica. La respuesta es que no siempre se tiene esta propiedad. Con el avance de la teoría veremos qué condiciones son necesarias para que $X / G$ sea una variedad algebraica.

Analicemos el caso en el que $X$ es una variedad algebraica afín con anillo de coordenadas R. Para que X/G sea una variedad algebraica afín debemos construirle su anillo de coordenadas. Como es habitual en matemáticas deseamos usar la información que ya poseemos, en este caso el álgebra de funciones de X.

Sin embargo nos topamos con el inconveniente de evaluar un polinomio $f \in R$ en $X / G$ puesto que depende del representante de la clase de equivalencia.

Esto nos motiva a dar la siguiente definición.

Definición 2.17. Sean $X, Y$ dos variedades algebraicas. Un morfismo $\phi: X \rightarrow Y$ se dice que es $\mathrm{G}$-invariante si $\phi(\mathrm{g} x)=\phi(x)$ para toda $\mathrm{g} \in \mathrm{G}$ y $x \in X$. También se dice que $\phi$ es constante en las órbitas.

En particular si $f \in R$ se dice que es G-invariante si $f(g \cdot x)=f(x)$ es decir, $g \cdot f=f$ para todo $g \in G$.

Denotemos por $R^{G}=\{f \in R \mid f$ es $G$-invariante $\}$

Como vemos, un buen candidato para el álgebra de funciones de $X / G$ es $R^{G}$ ya que para todo $f \in R^{G}$ se tiene que $\bar{f}: X / G \rightarrow K$ dada por $\bar{f}\left(\right.$ órb $\left._{G}(x)\right)=f(x)$ está bien definida.

El problema ahora es que no toda k-álgebra $R^{G}$ corresponde a una variedad algebraica, por ejemplo se requiere que $R^{G}$ sea de tipo finito o que no tenga elementos 
nilpotentes. Hilbert en su problema número catorce presenta la pregunta de si dada una álgebra $R$ finitamente generada y $G$ un grupo actuando en $R$ se tendrá que $R^{G}$ es finitamente generada. La respuesta es no, Nagata da un contraejemplo de ello.

Antes de proceder al caso general veamos unos ejemplos.

Ejemplo 2.18. Sea $G=\{-1,1\}$, este grupo finito actúa en $\mathbb{A}^{2}$

$$
\begin{aligned}
\mathrm{G} \times \mathbb{A}^{2} & \longrightarrow \mathbb{A}^{2} \\
(\lambda,(\mathrm{a}, \mathrm{b})) & \mapsto(\lambda \mathrm{a}, \lambda \mathrm{b}) .
\end{aligned}
$$

Para todo punto $(a, b)$ en $\mathbb{A}^{2}$, la órb $\{(a, b)\}$ consta de únicamente dos puntos. Esta acción tiene solamente un punto fijo, el cero. Esta acción se puede extender al anillo de coordenadas, entonces podemos calcular el anillo de funciones G-invariantes. Si $f \in k[x, y]^{G}$ entonces

$$
f(a, b)=f(-a,-b),
$$

es decir, $k[x, y]^{\mathrm{G}}$ está generado por las funciones pares, las funciones generadas por monomios cuadráticos, $x^{2}, x y, y^{2}$, entonces

$$
k[x, y]^{G}=k\left[x^{2}, x y, y^{2}\right] .
$$

Luego, la variedad afín asociada a $k\left[x^{2}, x y, y^{2}\right]$ son los ceros $V\left(u w-v^{2}\right)$. Entonces se tiene la siguiente biyección

$$
\begin{aligned}
\varphi: \mathbb{A}^{2} / G & \longrightarrow V\left(u w-v^{2}\right) \\
\operatorname{orb}_{G}(a, b) & \longmapsto\left(a^{2}, a b, b^{2}\right) .
\end{aligned}
$$

Por lo que podemos decir, que el espacio de órbitas, $\mathbb{A}^{2} / G$ es una variedad afín.

Ejemplo 2.19. $\mathbb{G}_{m}$ actúa en $\mathbb{A}^{4}$

$$
\begin{aligned}
\mathbb{G}_{\mathrm{m}} \times \mathbb{A}^{4} & \longrightarrow \mathbb{A}^{4} \\
(\lambda,(\mathrm{a}, \mathrm{b}, \mathrm{c}, \mathrm{d})) & \longmapsto\left(\lambda \mathrm{a}, \lambda \mathrm{b}, \lambda^{-1} \mathrm{c}, \lambda^{-1} \mathrm{~d}\right) .
\end{aligned}
$$

Sea $v=(a, b, c, d)$ en $\mathbb{A}^{4}$, órb $\mathbb{G}_{\mathfrak{m}}(v)$ es cerrado si $a, b, c, d \neq 0$, ya que para toda $\lambda$

$$
\lambda a \lambda b \lambda^{-1} c \lambda^{-1} d=a b c d .
$$

Si $f \in k[x, y, z, w]^{\mathbb{G}_{m}}$ entonces $f\left(\lambda a, \lambda b, \lambda^{-1} c, \lambda^{-1} d\right)=f(a, b, c, d)$, si

$f=\sum_{p, q, r, s} a_{p, q, r, s} x^{p} y^{q} z^{r} w^{s} \Longrightarrow f \cdot \lambda=\sum_{p, q, r, s} a_{p, q, r, s}(\lambda x)^{p}(\lambda y)^{q}\left(\lambda^{-1} z\right)^{r}\left(\lambda^{-1} w\right)^{s}$

Entonces $f$ es $\mathrm{G}$-inviariantes si y sólo si $(p+q)=(r+s)$. Dado que $p, q$ tienen el mismo signo, $p+q \neq 0$, por la misma razón, $(r+s) \neq 0$. De esta observación se conluye que $f$ no puede estar generado por monomios de la forma $x, y, z$ y $w$. Por otro lado, si $\mathrm{q}=0 \mathrm{y}(\mathrm{r}+\mathrm{s}) \neq 0$ se tiene que $\mathrm{f}$ puede estar generado por monomios $x z$ y $x w$. De manera análoga si $p=0, f$ puede estar generado por monomios de la forma $y z, y w$. Observe que $f$ no puede estar generado por monomios de la forma $x y, z w$ por la condición $(p+q)=(r+s)$. Por lo tanto $f$ está generado por los monomios $x z$, yw, $x w$ y $y z$. Es decir

$$
k[x, y, z, w]^{\mathbb{G}_{m}}=k[x z, y w, x w, y z] .
$$


Ahora bien, la variedad afín asociada a esta k-álgebra es $\mathrm{V}(\alpha \beta-\gamma \delta) \subset \mathbb{A}^{4}$. Entonces podemos definir el siguiente morfismo

$$
\begin{aligned}
\varphi: \mathbb{A}^{4} / \mathbb{G}_{m} & \longrightarrow \mathrm{V}(\alpha \beta-\gamma \delta) \\
\operatorname{órb}_{\mathbb{G}_{m}}(\mathrm{a}, \mathrm{b}, \mathrm{c}, \mathrm{d}) & \longmapsto(\mathrm{ac}, \mathrm{bd}, \mathrm{ad}, \mathrm{bc}) .
\end{aligned}
$$

El morfismo $\varphi$ tiene las siguientes propiedades

(a). $\varphi$ es suprayectiva. Sea $(a c, b d, a d, b d)$ en $V(\alpha \beta-\gamma \delta)$. Si alguna entrada es igual a cero, digamos la primera, $a c=0$, supongamos que $a=0$ entonces, $(0, b, c, d)$ en $\mathbb{A}^{4}$ satisface la condición bajo $\varphi$. Si todas las entradas son distintas de cero, $(a, b, c, d)$ pertenecen a $V(\alpha \beta-\gamma \delta)$ bajo $\varphi$.

(b). Si $p \in V(\alpha \beta-\gamma \delta) \backslash\{0\}$, entonces $\varphi^{-1}(p)$ contiene una sóla órbita, la cual es cerrada en $\mathbb{A}^{4}$. Dado que $p \neq 0$ existe al menos una entrada diferente de cero, sin pérdida de generalidad podemos suponer que es la primera entrada, es decir, $a c \neq 0$, lo cual implica que $a, c \neq 0$. Procedamos por casos, si $p$ solamente tiene una entrada distinta de cero, se tiene que $x=(a, 0, c, 0)$ pertenece a $\varphi^{-1}(p)$ y por lo tanto, órb $\mathbb{G}_{m}(x)$ también pertenece. Supongamos que existe otra órbita contenida en $\varphi^{-1}(p)$, sea $y=\left(a^{\prime}, 0, c^{\prime}, 0\right)$ un representante de dicha órbita. Ahora bien, $x$ y $y$ satisfacen que $a c=a^{\prime} c^{\prime}$ entonces $a / a^{\prime}=c^{\prime} / c$. Tomemos $\lambda=a / a^{\prime}=$ $c^{\prime} / c$, es claro que $\lambda \cdot x=y$ demostrando que pertenecen a la misma órbita. Ahora bien, supongamos que $p$ tiene al menos dos entradas distintas de cero, si $x \in \varphi^{-1}(p), x=(a, b, c, d)$ se tiene que al menos tres entradas de $x$ son distintas de cero, sin pérdida de generalidad podemos suponer que son $a, b$, c. Luego para toda $y \in \varphi^{-1}(p)$ con $y=\left(a^{\prime}, b^{\prime}, c^{\prime}, 0\right)$ podemos obtener la siguiente condición:

$$
\mathrm{a} / \mathrm{a}^{\prime}=\mathrm{c}^{\prime} / \mathrm{c}=\mathrm{b} / \mathrm{b}^{\prime}
$$

tomando $\lambda=a / a^{\prime}$ vemos que $\lambda \cdot y=x$, demostrando que están en la misma órbita. Por último, solo falta el caso en que $p$ tiene al menos dos entradas distintas de cero y $x \in \varphi^{-1}(p)$ tiene todas sus entradas distintas de cero. En esta caso, se obtiene la siguiente condición:

$$
\mathrm{a} / \mathrm{a}^{\prime}=\mathrm{c}^{\prime} / \mathrm{c}=\mathrm{b} / \mathrm{b}^{\prime}=\mathrm{d}^{\prime} / \mathrm{d},
$$

y al tomar $\lambda=a / a^{\prime}$ se demuestra que $\lambda \cdot y=x$.

Por lo tanto $\varphi^{-1}(p)$ consta de una sóla órbita. Dado que $\varphi$ es continua, $\varphi^{-1}(p)$ es cerrado en $\mathbb{A}^{4}$.

(c).

$$
\varphi^{-1}(0)=\left\{(\mathrm{a}, \mathrm{b}, 0,0) \mid(\mathrm{a}, \mathrm{b}) \in \mathbb{A}^{2}\right\} \cup\left\{(0,0, \mathrm{c}, \mathrm{d}) \mid(\mathrm{c}, \mathrm{d}) \in \mathbb{A}^{2}\right\} .
$$

La imagen inversa del cero contiene una infinidad de órbitas

(d). El punto fijo $0 \in \mathbb{A}^{4}$ genera la única órbita cerrada en la imagen inversa de $\varphi$. Consideremos la siguiente órbita, $v=(a, b, 0,0)$ con $a, b \neq 0$

$$
\operatorname{órb}_{\mathbb{G}_{\mathfrak{m}}}(v)=\left\{\lambda \cdot v=(\lambda a, \lambda b, 0,0) \mid \lambda \in k^{*}\right\},
$$


denotemos por $Y=$ órb $_{\mathbb{G}_{m}}(v)$. Dado que $\varphi$ es continua, se tiene que $\bar{Y} \in \varphi^{-1}(0)$. Por otro lado, $0, \bar{Y}$ contenidos en $\varphi^{-1}(0)$ implica que

$$
\overline{0} \cap \bar{Y} \neq \emptyset \Longrightarrow 0 \in \bar{Y} \text {. }
$$

Si fuese $Y=\bar{Y}$ se tendría que $0 \in Y$ lo cual no es posible, puesto que $\lambda \in k^{*}$. Por lo tanto $Y$ no es cerrado. Demostrando que la imagen inversa del cero bajo $\varphi$ contiene solamente una órbita cerrada, la del cero. Por lo que podemos tener la siguiente biyección

$$
\left\{\mathbb{G}_{m}-\text { órbitas cerradas }\right\} \simeq \mathrm{V}(\alpha \beta-\gamma \delta) .
$$

El siguiente ejemplo permite visualizar lo versátil que puede ser el área de los espacios cocientes.

Ejemplo 2.20. Sea $\mathbb{G}_{m}$ actuando en $\mathbb{A}^{2}$

$$
\begin{aligned}
\mathbb{G}_{\mathrm{m}} \times \mathbb{A}^{2} & \longrightarrow \mathbb{A}^{2} \\
(\mathrm{t},(\mathrm{a}, \mathrm{b})) & \longmapsto\left(\mathrm{ta}, \mathrm{t}^{-1} \mathrm{~b}\right)
\end{aligned}
$$

Para esta acción se tiene tres tipos de órbitas:

(a). Supongamos $x, y \neq 0$, entonces,

$$
\text { órb }_{\mathbb{G}_{\mathfrak{m}}}(x, y)=\left\{\left(t x, t^{-1} y\right) \mid t \in k^{*}\right\}=\{(a, b) \mid a b=x y=c t e\},
$$

es decir, las órbitas en este caso son unas hipérbolas, por lo tanto son órbitas cerradas.

(b). Sea $x=0$ y $y \neq 0$, entonces la órbita es

$$
\mathrm{U}=\text { orb }_{\mathbb{G}_{\mathrm{m}}}(0, \mathrm{y})=\left\{\left(0, \mathrm{t}^{-1} \mathrm{y}\right) \mid \mathrm{t} \in \mathrm{k}^{*}\right\},
$$

es decir, la recta $x=0$ menos el origen.

Sea $y=0, y x \neq 0$, en este caso

$$
\mathrm{V}=\text { órb }_{\mathbb{G}_{\mathfrak{m}}}(x, 0)=\left\{\left(\mathrm{t} x, 0 \mid \mathrm{t} \in \mathrm{k}^{*}\right\}\right.
$$

es la recta $y=0$ menos el origen.

Ambas órbitas son abiertas.

(c). El punto $(0,0)$ es un punto fijo, el cual es cerrado y

$$
0=\overline{\mathrm{U}} \cap \overline{\mathrm{V}} \text {. }
$$

Analicemos el caso en el que el álgebra de funciones G-invariantes es finitamente generada. En la siguiente proposición obtendremos algunas propiedades que son heredadas de la variedad $X$ al cociente.

Proposición 2.21. Sea $\mathrm{G}$ actuando en una variedad $\mathrm{X}$ con anillo de coordenadas $\mathrm{R}$ tal que $\mathrm{R}^{\mathrm{G}}$ es finitamente generado como $\mathrm{k}$-álgebra. Sea $\mathrm{Y}$ la variedad asociada $a \mathrm{R}^{\mathrm{G}}$. Si $\phi: \mathrm{X} \rightarrow \mathrm{Y}$ el morfismo inducido por la inclusión $\mathrm{R}^{\mathrm{G}} \subseteq \mathrm{R}$. Entonces:

(a). Dado cualquier diagrama

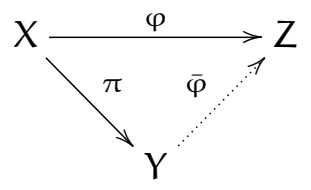


donde $\phi$ es un morfismo de variedades afines G-invariante. Entonces existe un único morfismo $\bar{\varphi}$ haciendo que el diagrama conmute, es de$\operatorname{cir} \bar{\varphi} \circ \pi=\varphi$.

(b). Si $\mathrm{X}$ es irreducible entonces $\mathrm{Y}$ es irreducible.

(c). Si $\mathrm{X}$ es normal entonces $\mathrm{Y}$ es normal.

Demostración. Sea $S$ el anillo de coordenadas de $Z$, dado que $\varphi: X \rightarrow Z$ entonces se induce un homomorfismo de anillos $\varphi^{*}: S \rightarrow R$. Sea $f \in S$ entonces para toda $g \in \mathrm{G}$ se tiene que

$$
\varphi^{*}(\mathrm{f}(\mathrm{gx}))=(\mathrm{f} \circ \varphi)(\mathrm{gx})=\mathrm{f}(\varphi(\mathrm{gx}))=\mathrm{f}(\varphi(\mathrm{x}))=(\mathrm{f} \circ \varphi)(\mathrm{x})=\varphi^{*}(\mathrm{f}(\mathrm{x})) .
$$

Por lo que $\varphi^{*}(S) \subset R^{G}$. Dado que $\pi$ es inducido por la inclusión, tenemos que el siguiente diagrama conmuta:

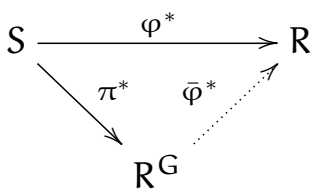

Lo cual implica que $\bar{\varphi} \circ \pi=\varphi$.

Para demostrar la unicidad, supongamos que existe $\psi$ que satisface el diagrama, es decir $\psi \circ \pi=\varphi$ lo cual implica $\pi^{*} \circ \psi^{*}=\varphi^{*}$. Como $\varphi^{*}$ se descompone de manera única se tiene que $\psi^{*}=\bar{\varphi}^{*} \Rightarrow \psi=\varphi$.

Para demostrar el inciso (b) como $R$ es dominio entero y $R^{G} \subset R$ se tiene que $\mathrm{R}^{\mathrm{G}}$ es dominio entero por tanto $\mathrm{Y}$ es irreducible.

Supongamos que $X$ es normal, por tanto $R$ es normal. Sea $K$ el campo de fracciones de $R^{G}$. Sea $f \in K$ entero sobre $R^{G}$. Entonces $f$ es entero sobre $R$ puesto que $R^{G} \subset R$ por lo tanto $f \in R$ por ser normal. Entonces $f \in K \cap R$. Ahora bien, $G$ actúa trivialmente en $\mathrm{K}$, puesto que si definimos la acción como:

$$
\begin{gathered}
\mathrm{G} \times \mathrm{K} \rightarrow \mathrm{K} \\
(\mathrm{g}, \mathrm{f} / \mathrm{h}) \rightarrow \mathrm{gf} / \mathrm{gh}
\end{gathered}
$$

Dado la relación de equivalencia de $K$ se tiene que $g p / g q \sim p / q$ ya que $p, q$ son $\mathrm{G}$-invariantes. Por lo que $\mathrm{R} \cap \mathrm{K}=\mathrm{R}^{\mathrm{G}}$ dado que $\mathrm{f}$ es $\mathrm{G}$-invariante. Con esto demostramos el inciso (c).

\subsection{Cocientes en geometría algebraica}

Como se vio en la sección anterior, cuando el anillo de polinomios G-invariantes es finitamente generado, la variedad asociada a dicho anillo cumple buenas propiedades heredadas de $X$ sin embargo faltan condiciones topológicas para dicho cociente. En esta sección daremos algunas definiciones de diversos tipos de cocientes que se tienen en geometría algebraica.

Definición 2.22. Sea $G$ un grupo algebraico actuando en una variedad $X$ y sea $\pi: X \rightarrow Y$ un morfismo entre variedades que es constante en órbitas. Entonces $\pi$ es un buen cociente categórico si: 
(a). Si $U \subset Y$ es abierto, entonces la función natural $\mathcal{O}_{Y}(\mathrm{U}) \longrightarrow \mathcal{O}_{X}\left(\pi^{-1}(\mathrm{U})\right)$ induce un isomorfismo

$$
\mathcal{O}_{\mathrm{Y}}(\mathrm{U}) \longrightarrow \mathcal{O}_{\mathrm{X}}\left(\pi^{-1}(\mathrm{U})\right)^{\mathrm{G}}
$$

(b). Si $W \subset X$ es cerrado y G-invariante, entonces $\pi(W) \subset Y$ es cerrado.

(c). Si $W_{1}, W_{2}$ son cerrados, disjuntos y G-invariantes, entonces $\pi\left(W_{1}\right)$ y $\pi\left(W_{2}\right)$ son disjuntos en $Y$.

La notación habitual, aunque no universal, para el buen cociente categórico es $\pi: X \rightarrow X / / G$

Esta definición nos permite dotar a $Y$ de una buena topología y nos describe cómo es su gavilla estructural a partir de la gavilla estructural de $X$. La idea consiste en imponer a la variedad $Y$ las condiciones que deseamos para que el espacio de órbitas, $\mathrm{X} / \mathrm{G}$ sea una variedad algebraica.

Para entender esta definición pensemos en el caso que estemos tratando con variedades afines. El hecho de pedir que $\pi$ sea constante en órbitas es similar a pensar a $\pi$ como la proyección natural de $X$ al cociente $X / G$.

En el inciso (a) nos indica que para un abierto en el cociente $X / G$, el anillo de funciones regulares en él estaría formado por las funciones regulares $\mathrm{G}$-invariantes en X. La razón de ello es que los polinomios que podríamos evaluar en $X / G$ son los G-invariantes, como se mostró en la sección anterior.

Mientras que el inciso (b) nos dice de manera intuitiva que si pensamos a $W$ como los ceros de un ideal I, $\bar{W}=\left\{\right.$ órb $\left._{\mathrm{G}}(x) \mid x \in W\right\} \subset X / G$ corresponde a los ceros del ideal $\left\langle\mathrm{I}^{\mathrm{G}}\right\rangle$ de ahí la razón de que $\pi(\mathrm{W})$ sea cerrado.

Por último la condición (c) se asegura que la topología en $X / G$ sea la deseada, la topología de Zariski. Es decir, si $W_{1}, W_{2}, \subset X$ cerrados $G$-invariantes tales que $W_{1} \cap W_{2} \neq \emptyset$, luego $W_{1} \cap W_{2}$ es cerrado en $X$. Sean $I_{1}, I_{2}$ los ideales de polinomios asociados tal que $W_{i}=V\left(I_{i}\right)$. Como la intersccción de dichos cerrados no es vacía se tiene que $I_{1} \cap I_{2} \neq \emptyset$. Por otro lado el análisis del inciso anterior nos permite pensar en que $\pi\left(\mathrm{W}_{\mathrm{i}}\right)=\mathrm{V}\left(\left\langle\mathrm{I}_{\mathrm{i}}{ }^{\mathrm{G}}\right\rangle\right)$ sin embargo se puede dar el caso de que $\left\langle\mathrm{I}_{1}{ }^{\mathrm{G}}\right\rangle \cap\left\langle\mathrm{I}_{2}{ }^{\mathrm{G}}\right\rangle=\emptyset$ lo cual genera incongruencias en la topología.

Cuando tenemos un buen cociente categórico de $\pi: X \longrightarrow X / / G$ nos permite demostrar de manera fácil unas propiedades de las órbitas en $X$ :

Observación 2.23. Sea $\pi: X \longrightarrow X / / G$ :

(a). Sea $p \in X / / G$ y sea $x \in X$ es tal que $\pi(x)=p$ entonces órb or $_{G}(x) \subset$ $\pi^{-1}(p)$ ya que $\pi^{-1}(p)$ es cerrado en $X$ entonces $W=\pi^{-1}(p) \cap \overline{\text { órb }} b_{G}(x)$ es cerrado que contiene a la órbita de $\mathrm{x}$ que a su vez está contenido en su cerradura por lo tanto $W=\overline{\text { orb }_{\mathrm{G}}(x)}$.

(b). En la observación 2.8, se demostró que la cerradura de una órbita es Ginvariante.

$$
\mathrm{G} \cdot \overline{\text { orb }_{\mathrm{G}}(x)} \subseteq \overline{\operatorname{orb}_{\mathrm{G}}(x)}
$$

A continuación enunciaremos un teorema sobre las propiedades que tiene un buen cociente categórico

Teorema 2.24. Sea $\pi: X \rightarrow X / / G$ un buen cociente categórico. Entonces: 
(a). Dado cualquier diagrama:

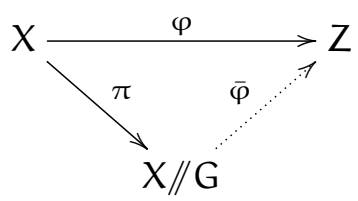

donde $\varphi$ es un morfismo tal que $\varphi(\mathrm{g} \cdot \mathrm{x})=\varphi(\mathrm{x})$ para todo $\mathrm{g} \in \mathrm{G} y$ $\chi \in X$, existe un único morfismo $\bar{\varphi}$ haciendo que el diagrama conmute, es decir, $\bar{\varphi} \circ \pi=\varphi$.

(b). $\pi$ es suprayectiva.

(c). Un subconjunto $\mathrm{U} \subset \mathrm{X} / / \mathrm{G}$ es abierto si y solo si $\pi^{-1}(\mathrm{U}) \subset \mathrm{X}$ es abierto.

(d). Si $\mathrm{U} \subset \mathrm{X} / / \mathrm{G}$ es abierto y no vacío, entonces $\pi_{\mid \pi^{-1}(\mathrm{U})}: \pi^{-1}(\mathrm{U}) \rightarrow \mathrm{U}$ es un buen cociente categórico.

(e). Para cualesquiera dos puntos $\mathrm{x}, \mathrm{y} \in \mathrm{X}$ se tiene que

$$
\pi(x)=\pi(y) \Longleftrightarrow \overline{\operatorname{orb}_{\mathrm{G}}(\mathrm{x})} \cap \overline{\operatorname{orb}_{\mathrm{G}}(\mathrm{y})} \neq \emptyset
$$

Demostración. Para demostrar el inciso (a) y (b) necesitaremos del siguiente razonamiento.

Sea $\left\{U_{i}\right\}_{i}$ una cubierta abierta afín de $X / / G$. Como $\pi$ es continua se tiene que $\pi^{-1}\left(\mathrm{U}_{i}\right)$ es un abierto, sin embargo no necesariamente es afín, en ese caso tomemos una cubierta abierta afín de $\pi^{-1}\left(U_{i}\right)$ y restringirnos a un abierto afín de $\pi^{-1}\left(\mathrm{U}_{\mathrm{i}}\right)$.

Iniciemos demostrando el inciso (b). Dado que para cualquier $\mathrm{U} \subset \mathrm{X} / / \mathrm{G}$ abierto se tiene un morfismo inyectivo entre $\mathcal{O}_{Y}(\mathrm{U}) \rightarrow \mathcal{O}_{X}\left(\pi^{-1}(\mathrm{U})\right)$ ya que $\mathcal{O}_{Y}(\mathrm{U})=$ $\mathcal{O}_{X}\left(\pi^{-1}(\mathrm{U})\right)^{\mathrm{G}}$ lo cual implica que el morfismo $\pi$ es dominante. Aquí usamos que un morfismo es dominante, entre $\varphi: X \rightarrow Y$, si y solo si $\varphi^{*} \mathrm{~K}[\mathrm{Y}] \rightarrow \mathrm{K}[\mathrm{X}]$ es inyectivo, ver [12]. Por lo tanto la imagen de $\pi$ es densa en $X / / G$. Ahora bien, $X$ es cerrado e invariante bajo $G$ obviamente, por lo tanto $\pi(X)$ es cerrado y dado que también es denso en $X / / G$, se tiene que $\pi(X)=\overline{\pi(X)}=X / / G$ con ello se demuestra que $\pi$ es suprayectivo.

Para demostrar el inciso (a), sea $\left\{V_{i}\right\}_{i \in I}$ una cubierta abierta de Z. Definimos $W_{i}=X \backslash \varphi^{-1}\left(V_{i}\right)$ cerrado en $X$. También tenemos que $W_{i}$ es $G$-invariante lo cual implica que $\pi\left(W_{i}\right)$ es cerrado. Ahora definamos $U_{i}=(X / / G) \backslash\left(\pi\left(W_{i}\right)\right)$ abierto. Por construcción de los abiertos $U_{i}, V_{i}$ se tiene que $\pi^{-1}\left(U_{i}\right) \subset \varphi^{-1}\left(V_{i}\right)$. Por otro lado $W=\bigcap_{i} W_{i}=\emptyset$, puesto que si existe $x \in W \Rightarrow x \notin \varphi^{-1}\left(V_{i}\right)$ para toda $i$, entonces $\varphi(x) \notin V_{i} \forall i$ contradiciendo que $\left\{V_{i}\right\}_{i \in I}$ es cubierta de $Z$. Por lo tanto $W=\emptyset$.

Por la propiedad (c) de la definición de buen cociente categórico se tiene que $\bigcap_{i} \pi\left(W_{i}\right)=\emptyset$ esto implica que $Y=\bigcup_{i} U_{i}$, son una cubierta abierta de $Y$.

Ahora podremos definir un homomorfismo $\varphi^{*}: \mathcal{O}\left(V_{i}\right) \rightarrow \mathcal{O}\left(\varphi^{-1}\left(V_{i}\right)\right)^{G}$ dado de la siguiente manera: para toda $f \in \mathcal{O}\left(V_{i}\right)$,

$$
\varphi^{-1}\left(\mathrm{~V}_{\mathrm{i}}\right) \stackrel{\varphi}{\longrightarrow} \mathrm{V}_{\mathrm{i}} \stackrel{\mathrm{f}}{\longrightarrow} \mathrm{k} \quad \varphi_{\mathrm{i}}(\mathrm{f})=\mathrm{f} \circ \varphi
$$


Como $\varphi$ es constante en órbitas se tiene que $\varphi^{*}(f)(g x)=(f \circ \varphi)(g x)=$ $f(\varphi(g x))=f(\varphi(x))=\varphi^{*}(f)$ es decir $\varphi^{*}(f)$ es G-invariante. Por lo tanto $\operatorname{Im} \varphi^{*}=$ $\mathcal{O}\left(\varphi^{-1}\left(V_{i}\right)\right)^{G}$ tal como se quería.

Ahora bien, dado que $\pi^{-1}\left(\mathrm{U}_{i}\right) \subset \varphi^{-1}\left(\mathrm{~V}_{i}\right)$, por medio de la inclusión j:

$$
\pi^{-1}\left(\mathrm{U}_{i}\right)^{-1} \stackrel{\mathrm{J}}{\longrightarrow} \varphi^{-1}\left(\mathrm{~V}_{\mathrm{i}}\right) \stackrel{\mathrm{f}}{\longrightarrow} \mathrm{k}
$$

podemos definir un homomorfismo de:

$$
\left.\mathcal{O}\left(\varphi^{-1}\left(\mathrm{~V}_{\mathrm{i}}\right)\right) \stackrel{\mathrm{j}^{*}}{\longrightarrow} \mathcal{O}\left(\pi^{-1}\left(\mathrm{U}_{\mathrm{i}}\right)\right)^{\mathrm{G}} \quad \mathcal{j}^{*}(\mathrm{f})=\mathrm{f} \circ\right)
$$

Abusando de la notación, esto nos define un morfismo de restricción:

$$
\mathcal{O}\left(\varphi^{-1}\left(\mathrm{~V}_{i}\right)\right)^{\mathrm{G}} \stackrel{\mathrm{j}^{*}}{\longrightarrow} \mathcal{O}\left(\pi^{-1}\left(\mathrm{U}_{i}\right)\right)^{\mathrm{G}}
$$

nótese que el contradominio es el correcto puesto que j es inyectivo.

Por la propiedad (a) del buen cociente categórico $\mathcal{O}\left(\varphi^{-1}\left(\mathrm{U}_{i}\right)\right)^{\mathrm{G}} \simeq \mathcal{O}\left(\pi^{-1}\left(\mathrm{U}_{i}\right)\right)$ isomorfimos inducido por $\pi^{*}: \mathcal{O}\left(\mathrm{U}_{i}\right) \longrightarrow \mathcal{O}\left(\pi^{-1}\left(\mathrm{U}_{i}\right)\right)$. Entonces hemos construido el siguiente diagrama conmutativo:

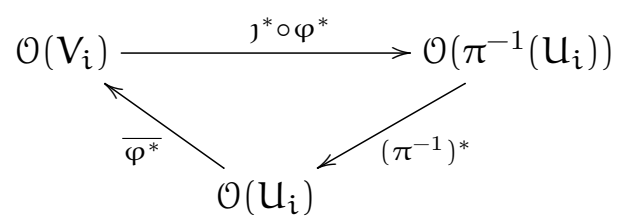

Dado que estamos trabajando en el caso afín, $\overline{\varphi^{*}}$ induce un morfismo $\bar{\varphi}$ : $\mathrm{U}_{i} \longrightarrow \mathrm{V}_{i}$ que hace conmutar el siguiente diagrama:

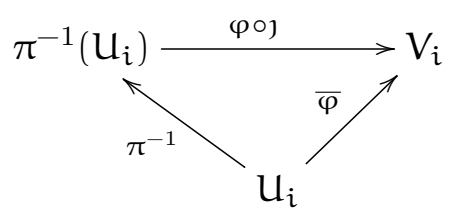

Como se ve, la función $\bar{\varphi}$ ha sido definida localmente, para hacerlo de modo global es necesario verificar en las intersecciones de abiertos $U_{i}, U_{j}$ que $\bar{\varphi}$ esté bien definida o bien si hacemos el análisis en los anillos de funciones regulares es decir, se necesita verificar que $\overline{\varphi^{*}}$ esté bien definida en la intersección de abiertos $V_{i}, V_{j}$ y esto se comprueba fácilmente ya que $\overline{\varphi^{*}}$ ha sido definida por la composición de restricciones de funciones globales en las cuales dichas funciones están bien definidas en las intersecciones de los abiertos. Por lo tanto queda demostrado la existencia

Para demostrar que $\bar{\varphi}$ es único supongamos que existe $\psi$ que también hace conmutar el diagrama, es decir $\bar{\varphi} \circ \pi=\varphi=\psi \circ \pi$, como $\pi$ es suprayectivo: para todo $y \in X / / G$ existe $x \in X$ tal que $\pi(x)=y$ entonces

$$
\bar{\varphi}(y)=\bar{\varphi}(\pi(x))=\varphi(x)=\psi(\pi(x))=\psi(y) .
$$

Por lo tanto $\bar{\varphi}$ es único. 
(c): Un lado de la implicación es sencilla, dado que $\pi$ es continua, dado un abierto $\mathrm{U} \subset \mathrm{X} / / \mathrm{G}$ se tiene que $\pi^{-1}(\mathrm{U})$ es abierto en $\mathrm{X}$.

Para la otra implicación consideremos $\mathrm{U} \subset \mathrm{X}$ abierto y denotemos por $\mathrm{V}=$ $X \backslash U$ el conjunto cerrado correspondiente. Primero demostremos el caso cuando $\mathrm{V}$ es G-invariante, o bien, $\mathrm{U}$ es $\mathrm{G}$-invariante. Dado que $\mathrm{V}$ es cerrado y $\mathrm{G}$ invariante, se tiene que $\pi(\mathrm{V})$ es cerrado, por definición de buen cociente. Por lo que $(\mathrm{X} / / \mathrm{G}) \backslash \pi(\mathrm{V})$ es abierto. Dado que $\pi$ es sobreyectiva, se tiene que

$$
\pi(\mathrm{U})=(\mathrm{X} / / \mathrm{G}) \backslash \pi(\mathrm{V})
$$

demostrando que $\pi(\mathrm{U})$ es abierto.

Ahora bien, analicemos el caso cuando $\mathrm{V}$ no es $\mathrm{G}$-invariante. Entonces $\mathrm{V}^{\mathrm{G}} \subset$ $V$, sin embargo $V^{G}$ no es necesariamente cerrado, por lo que consideremos la cerradura

$$
\mathrm{V}^{\mathrm{G}} \subset \overline{\mathrm{V}^{\mathrm{G}}} \subset \mathrm{V}
$$

Por otro lado, $\overline{V^{G}}$ es G-invariante, por lo que podemos asumir desde un inicio que $V^{G}$ es cerrado. Consideremos el abierto $U^{\prime}=X \backslash V^{G}$, el cual es $G$-invariante. Por lo demostrado en el caso anterior, $\pi(\mathrm{U})$ es abierto. Por otro lado, para toda $x \in \mathrm{U}^{\prime} \backslash \mathrm{U}$ se cumple que órb $\mathrm{G}_{\mathrm{G}}(\mathrm{x}) \cap \mathrm{U} \neq \emptyset$, puesto que de no ser así se tendría que órb $\mathrm{G}_{\mathrm{G}}(\mathrm{x}) \subset \mathrm{U}^{\prime} \backslash \mathrm{U}$ lo cual implicaría órb $\mathrm{G}(\mathrm{x}) \subset \mathrm{V}$, entonces órb $\mathrm{G}_{\mathrm{G}}(\mathrm{x}) \subset \mathrm{V}^{\mathrm{G}}$ contradiciendo el hecho de que $\mathrm{U}^{\prime} \cap \mathrm{V}^{\mathrm{G}}=\emptyset$. Entonces, dado que $\pi$ es constante en órbitas se tiene que $\pi(\mathrm{U})=\pi\left(\mathrm{U}^{\prime}\right)$. Por lo tanto $\pi(\mathrm{U})$ es abierto.

(d) Sea $U \subset X / / G$ abierto. Sea $V \subset U$ abierto en la topología relativa. Entonces existe $W \subset X / / G$ tal que $V=W \cap U$ de aquí que $V$ es abierto en $X / / G$. Por otro lado $\pi^{-1}(\mathrm{U})$ y $\pi^{-1}(\mathrm{~V})$ son abiertos en $X$ por el inciso (c). Entonces tenemos la siguiente implicación ya que $\pi^{-1}(\mathrm{~V}) \subset \pi^{-1}(\mathrm{U})$ :

$$
\mathcal{O}_{\mathrm{X} / / \mathrm{G}}(\mathrm{V}) \simeq \mathcal{O}_{\mathrm{X}}\left(\pi^{-1}(\mathrm{~V})\right)^{\mathrm{G}} \Longrightarrow \mathcal{O}_{\mathrm{u}}(\mathrm{V}) \simeq \mathcal{O}_{\pi^{-1}(\mathrm{u})}\left(\pi^{-1}(\mathrm{~V})\right)^{\mathrm{G}}
$$

Demostrememos la propiedad (b) de la definición. Sea $V \in \pi^{-1}(\mathrm{U})$ cerrado y G-invariante. Entonces $\exists W \subset X$ tal que $V=W \cap \pi^{-1}(\mathrm{U})$. Por la invarianza de $\mathrm{V}$ se tiene que $\mathrm{V} \subset \overline{W^{G}}$. De aquí que $V=\overline{W^{G}} \cap \pi^{-1}(\mathrm{U})$. Aplicando $\pi$ a $V$ se tiene $\pi(\mathrm{V})=\pi\left(\overline{W^{G}}\right) \cap \mathrm{U}$. Por la segunda propiedad del buen cociente categórico se tiene que $\pi\left(\overline{W^{G}}\right)$ es cerrado, por lo tanto $\pi(\mathrm{V})$ es cerrado.

Sean $V_{1}, V_{2} \subset \pi^{-1}(U)$ cerrados y $G$-invariantes y $V_{1} \cap V_{2}=\emptyset$. Como se vio en el párrafo anterior existen $W_{1}, W_{2} \subset X$ tales que $V_{i}=\overline{W_{i}^{G}} \cap \pi^{-1}(U)$. Además se tiene que $\overline{W_{1}^{\mathrm{G}}} \cap \overline{W_{2}^{\mathrm{G}}}=\emptyset$. Entonces:

$$
\begin{aligned}
\pi\left(\mathrm{V}_{1}\right) \cap \pi\left(\mathrm{V}_{2}\right) & =\pi\left(\overline{\mathrm{W}_{1}^{\mathrm{G}}} \cap \pi^{-1}(\mathrm{U})\right) \cap \pi\left(\overline{\mathrm{W}_{2}^{\mathrm{G}}} \cap \pi^{-1}(\mathrm{U})\right) \\
& =\pi\left(\overline{\mathrm{W}_{1}^{\mathrm{G}}}\right) \cap \mathrm{U} \cap \pi\left(\overline{\mathrm{W}_{2}^{\mathrm{G}}}\right) \cap \mathrm{U} \\
& =\pi\left(\overline{\mathrm{W}_{1}^{\mathrm{G}}}\right) \cap \pi\left(\overline{\mathrm{W}_{2}^{\mathrm{G}}}\right) \cap \mathrm{U} \\
& =\emptyset
\end{aligned}
$$

ya que $\pi\left(\overline{W_{1}^{G}}\right) \cap \pi\left(\overline{W_{2}^{G}}\right)=\emptyset$ por ser $\pi$ buen cociente categórico.

Con esto se demuestra que la propiedad de ser buen cociente categórico es una propiedad local. 
(e) Sean $x, y \in X$ tales que $\pi(x)=\pi(y)$, por demostrar que órb $\bar{G}(x) \cap$ $\overline{\text { orb }_{\mathrm{G}}(\mathrm{y})} \neq \emptyset$.

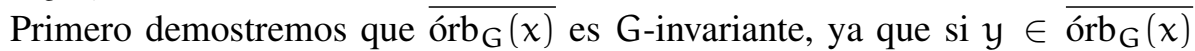
se tiene que para toda $g \in \mathrm{G} \pi(\mathrm{gy})=\pi(\mathrm{y}) \in \pi\left(\overline{\text { orb }_{\mathrm{G}}(\mathrm{x})}\right)$ esto implica que gy $\in \pi^{-1}\left(\overline{\text { órb }_{\mathrm{G}}(x)}\right) \subset \overline{\text { órb }_{\mathrm{G}}(x)}$.

Ahora bien, supongamos que órb $\mathrm{rb}_{\mathrm{G}}(x) \cap \overline{\text { ób }_{\mathrm{G}}(\mathrm{y})}=\emptyset$, esto implica que $\pi\left(\overline{\text { orb }_{\mathrm{G}}(x)}\right) \cap$ $\pi\left(\overline{\text { orb }_{\mathrm{G}}(\mathrm{y})}\right)=\emptyset$ pero ambos contienen al punto $\pi(\mathrm{x})=\pi(\mathrm{y})$.

Para demostrar la otra implicación supongamos que $\pi(x) \neq \pi(y)$ entonces $\pi^{-1}(x)$ y $\pi^{-1}(y)$ son cerrados disjuntos. Dado que órb ${ }_{\mathrm{G}}(x) \subset \overline{\text { órb }_{\mathrm{G}}(x)} \subset \pi^{-1}(x)$ y órb or $_{\mathrm{G}}(\mathrm{y}) \overline{\text { órb }_{\mathrm{G}}(\mathrm{y})} \subset \pi^{-1}(\mathrm{y})$ se tiene que órb or $_{\mathrm{G}}(x) \cap \overline{\text { orb }_{\mathrm{G}}(y)}=\emptyset$.

En la sección 2.1 se da una propiedad que tienen las órbitas, en la preposición que sigue se da una relación entre las órbitas y el buen cociente categórico.

Proposición 2.25. Sea $\mathrm{G}$ un grupo algebraico afín actuando en una variedad algebraica $\mathrm{X}$ y supongamos que existe un buen cociente categórico $\pi: \mathrm{X} \longrightarrow \mathrm{X} / / \mathrm{G}$. Entonces:

(a). Si $\mathrm{p} \in \mathrm{X} / / \mathrm{G}$, entoncen $\pi^{-1}(\mathrm{p})$ contiene una única órbita cerrada.

(b). $\pi$ induce una biyección

$\{$ órbitas cerradas de $\mathrm{X}\} \simeq \mathrm{X} / / \mathrm{G}$.

Demostración. Inciso (a): Sea $p \in X / / G$ y sea $G_{e}$ la componente de la identidad, definida en la sección 1 , dado que $G_{e}$ es una componente conexa (proposición 1.10) se tiene que $\pi^{-1}(p)$ es invariante bajo $G_{e}$. Por lo que podemos tomar una órbita órb $\mathrm{G}_{e}(x) \subset \pi^{-1}(p)$ con la condición de que órb $\mathrm{G}_{e}(x)$ tenga dimensión mínima.

Por otro lado órb $\mathrm{G}_{e}(x)$ es irreducible en $\mathrm{G}_{e}$ ya que este es una componente irreducible (proposición 1.10). Como órb $\mathrm{G}_{e}(\mathrm{x})$ es construible (por Chevalley y teorema 2.9) existe un abierto no vacío $U$ de $G_{e}$ tal que $U \subset$ órb $G_{e}(x)$ que es denso. Supongamos que órb $\mathrm{G}_{e}(\mathrm{x})$ no es cerrado, entonces existe $y \in X$ tal que $\overline{\text { órb }_{\mathrm{G}_{e}}(\mathrm{x})}$ contiene una órbita de $y$ que no intersecta a la órbita de $x$. Por lo que:

$$
\overline{\text { orb }_{\mathrm{G}_{e}}(\mathrm{y})} \subset \overline{\text { orb }_{\mathrm{G}_{e}}(x)}-\text { órb }_{\mathrm{G}_{e}}(x) \subset \overline{\text { orb }_{\mathrm{G}_{e}}(x)}-\mathrm{U} \text {. }
$$

Por otro lado órb $\mathrm{G}_{e}(x)$ es irreducible eso implica que :

$$
\operatorname{dim}\left(\overline{\text { órb }_{\mathrm{G}_{e}}(x)}-\mathrm{U}\right)<\operatorname{dim}\left(\overline{\text { órb }_{\mathrm{G}_{e}}(x)}\right)
$$

implicando que órb $\mathrm{G}_{e}(\mathrm{y})$ tiene dimensión menor a órb $\mathrm{G}_{e}(\mathrm{x})$ y esto contradice la suposición de que órb $\mathrm{G}_{e}(x)$ tiene dimensión mínima. Por lo tanto órb $\mathrm{G}_{e}(x)$ es cerrado.

Como $\overline{\mathrm{G}_{e}}$ es de índice finito en $\mathrm{G}$ existen $\mathrm{g}_{1}, \mathrm{~g}_{2}, \ldots, \mathrm{gt}$ tales que :

$$
\operatorname{órb}_{G}(x)=\bigcup_{i=1}^{t} g_{i} \cdot G_{e}
$$

demostrando que la órbita de x es cerrada. 
Para demostrar la unicidad supongamos que existen dos órbitas cerradas órb $\mathrm{G}_{\mathrm{G}}(\mathrm{x})$, órb $_{\mathrm{G}}(\mathrm{y})$, contenidas en $\pi^{-1}(p)$, entonces por la propiedad (e) del teorema anterior:

$\emptyset \neq \overline{\text { órb }_{\mathrm{G}}(x)} \cap \overline{\text { órb }_{\mathrm{G}}(y)}=\operatorname{orb}_{\mathrm{G}}(x) \cap$ órb $_{\mathrm{G}}(\mathrm{y}) \Longrightarrow$ órb $_{\mathrm{G}}(x)=$ órb $_{\mathrm{G}}(\mathrm{y})$.

Inciso (b): Sean órb $\mathrm{G}_{\mathrm{G}}(\mathrm{x})$ y órb $\mathrm{r}_{\mathrm{G}}(\mathrm{y})$ dos órbita cerradas y distintas en $X$, entonces son órbitas disjuntas y por la propiedad (e) del teorema anterior se tiene que $\pi\left(\right.$ órb $\left._{\mathrm{G}}(\mathrm{x})\right) \neq \pi\left(\right.$ órb $\left._{\mathrm{G}}(\mathrm{y})\right)$ por lo tanto órbitas distintas caen en puntos distintos en $X / / G$. Por la suprayectividad de $\pi$ se tiene que a cada punto de $X / / G$ corresponde una órbita y por el inciso anterior de este mismo lema se concluye que le corresponde una órbita cerrada.

Por lo tanto existe una correspondencia biyectiva tal como se quería.

La siguiente pregunta natural que surge del teorema anterior, es que propiedades se obtienen cuando las órbitas de la acción son cerradas, en un buen cociente categórico. El siguiente teorema nos da información en tal caso.

Proposición 2.26. Sea $\pi: X \longrightarrow X / / G$ es un buen cociente categórico. Entonces las siguientes condiciones son equivalentes:

(a). Todas las órbitas son cerradas en X.

(b). Dados dos puntos $x, y$ en $X$ se tiene que:

$$
\pi(\mathrm{x})=\pi(\mathrm{y}) \Longleftrightarrow \mathrm{x} \sim \mathrm{y} .
$$

Es decir, $x, y$ pertenecen a la misma órbita.

(c). $\pi$ induce una biyección

$$
\{\text { órbitas en } \mathrm{X}\} \simeq \mathrm{X} / / \mathrm{G} \text {. }
$$

(d). La imagen del morfismo $\sigma: \mathrm{G} \times \mathrm{X} \longrightarrow \mathrm{X} \times \mathrm{X}$ definido por $(\mathrm{g}, \mathrm{X}) \mapsto$ ( $\mathrm{g} x, \mathrm{X})$ es el producto fibrado $\mathrm{X} \times{ }_{\mathrm{X} / / \mathrm{G}} \mathrm{X}$.

Demostración. Iniciemos demostrando (a) implica (b). Sean $x, y$ en $X$ tales que $\pi(x)=\pi(y)$. Entonces por el inciso (e) del Teorema 2.24 se tiene:

$\emptyset \neq \overline{\text { órb }_{\mathrm{G}}(x)} \cap \overline{\text { órb }_{\mathrm{G}}(\mathrm{y})}=$ órb $_{\mathrm{G}}(x) \cap$ órb $_{\mathrm{G}}(\mathrm{y}) \Longrightarrow$ órb $_{\mathrm{G}}(\mathrm{x})=$ órb $_{\mathrm{G}}(\mathrm{y}) \Longrightarrow x \sim \mathrm{y}$

El otro lado de la implicación del inciso (b) es por definición de órbita.

$(b) \Rightarrow$ (c) Sean dos órbitas de $G$ en $X$ tales que $\pi\left(\right.$ órb $\left._{\mathrm{G}}(\mathrm{x})\right)=\pi\left(\right.$ órb $_{\mathrm{G}}(\mathrm{y})$ ), por hipótesis se tiene que órb $\mathrm{G}(\mathrm{x})=\operatorname{oorb}_{\mathrm{G}}(\mathrm{y})$ por lo tanto $\pi$ es inyectiva. Luego por el inciso (b) del teorema 2.24 tenemos que $\pi$ es sobreyectiva, con lo cual concluimos que $\pi$ es una biyección.

(b) $\Rightarrow$ (d) Por definición del producto fibrado tenemos:

$$
X \times{ }_{X / / G} X=\{(y, x) \in X \times X \mid \pi(x)=\pi(y)\}=\{(y, x) \in X \times X \mid x \sim y\}
$$

Esta última igualdad es obtenida de la hipótesis, por lo que existe $g \in \mathrm{G}$ tal que $\mathrm{gx}=\mathrm{y}$ por lo tanto

$X \times{ }_{X / / G} X=\{(y, x) \in X \times X \mid y=g x\}=\{(g x, x) \in X \times X \mid g \in G, x \in X\}=\operatorname{Im} \sigma$

(d) $\Rightarrow$ (a) Sea $x \in X$ y $y \in \overline{\text { orb }_{G}(x)}$, demostremos que $y \in$ órb $_{G}(x)$. Dado que $y \in \overline{\text { orb }_{\mathrm{G}}(\mathrm{x})}$ se tiene que $\pi(\mathrm{y})=\pi(\mathrm{x})$ por lo que $(\mathrm{y}, \mathrm{x}) \in \mathrm{X} \times \mathrm{X} / / \mathrm{X}=\operatorname{Im} \sigma$ por lo que existe $g \in G$ tal que $(y, x)=(g x, x)$ lo cual implica $y=g x$ por lo 
tanto $y \in$ órb $_{\mathrm{G}}(x)$ es decir órb o $_{\mathrm{G}}(\mathrm{x}) \subseteq$ órb $_{\mathrm{G}}(\mathrm{x})$. Con lo cual se demuestra que las órbitas son cerradas.

En el caso de un buen cociente categórico satisfaga la proposición anterior se dice que es un buen cociente geométrico. La notación para estos cocientes es $\pi: X \longrightarrow X / G$.

Teorema 2.27. Sea $\pi: X \longrightarrow X / / G$ un buen cociente categórico. Entonces son equivalentes:

(a). $\mathrm{X}$ contiene un abierto de Zariski denso $\mathrm{G}$-invariante, $\mathrm{U}_{0}$, tal que órb $\mathrm{G}_{\mathrm{G}}(\mathrm{x})$ es cerrado en $\mathrm{X}$ para todo $\mathrm{X} \in \mathrm{U}_{0}$.

(b). $\mathrm{X} / / \mathrm{G}$ tiene un abierto denso de Zariski, $\mathrm{U}$, tal que

$$
\pi_{\mid \pi^{-1}(\mathrm{u})}: \pi^{-1}(\mathrm{U}) \longrightarrow \mathrm{u}
$$

es un buen cociente categórico.

Demostración. Demostremos a) implica b). Existe $\mathrm{U}_{0} \subset \mathrm{X}$ un abierto denso $\mathrm{G}$-invariante. Denotemos por $\mathrm{W}=X \backslash \mathrm{U}_{0}$ el cerrado $\mathrm{G}$-invariante correspondiente. Luego para $x \in \mathrm{U}_{0}$ la órb $\mathrm{G}(\mathrm{x}) \subset \mathrm{U}_{0}$ es cerrado y $\mathrm{G}$-invariante, lo cual implica que $\pi(x)=\pi\left(\right.$ órb $\left._{\mathrm{G}}(x)\right) \notin W$ por ser $\pi$ buen cociente categórico. Por lo que $X / / G=\pi\left(\mathrm{U}_{0}\right) \cup \pi(W)$. Sea $U=\pi\left(\mathrm{U}_{0}\right)$, dado que $W$ es cerrado y $G$ invariante, $\pi(W)$ es cerrado, entonces $U$ es abierto. Por hipótesis $U_{0}$ es denso, lo cual implica que $\pi\left(\mathrm{U}_{0}\right)=\mathrm{U}$ es denso. Por ser $\pi$ un buen cociente categórico se tiene que

$$
\pi_{\mid \mathrm{U}_{0}}: \mathrm{U}_{0} \longrightarrow \mathrm{U}
$$

es un buen cociente categórico y por hipótesis todas las órbitas en $\mathrm{U}_{0}$ son cerradas concluyendo que

$$
\pi_{\mid \mathrm{U}_{0}}: \mathrm{U}_{0} \longrightarrow \mathrm{U}
$$

es un buen cociente geométrico.

Para la demostración restante observe que si $X$ es una G-variedad y $\varphi: X \longrightarrow$ $Y$ es un morfismo constante en G-órbitas entre variedades, se cumple que para todo subconjunto $\mathrm{U}$ de $\mathrm{Y}, \varphi^{-1}(\mathrm{U})$ es un subconjunto $\mathrm{G}$-invariante. Por lo que en el caso de nuestro interés, si $U \in X / / G$ es abierto y denso, se tiene que $\pi^{-1}(U)$ es un abierto denso G-invariante. Por ser

$$
\pi_{\mid \pi^{-1}(\mathrm{U})}: \pi^{-1}(\mathrm{U}) \longrightarrow \mathrm{U}
$$

buen cociente geométrico, las órbitas contenidas en $\pi^{-1}(\mathrm{U})$ son cerradas, concluyendo así la demostación de la proposición.

Teorema 2.28. Sea $\mathrm{G}$ un grupo algebraico que actúa en $\mathrm{X}$ y $\pi: \mathrm{X} \longrightarrow \mathrm{Y}$ un morfismo de variedades que es constante en G-órbitas. Supongamos que existe una cubierta de $\mathrm{Y}, \mathrm{Y}=\cup_{\gamma} \mathrm{V}_{\alpha}$ tal que

$$
\pi_{\mid \pi^{-1}\left(\mathrm{~V}_{\gamma}\right)}: \pi^{-1}\left(\mathrm{~V}_{\gamma}\right) \longrightarrow \mathrm{V}_{\gamma}
$$

es un buen cociente categórico para toda $\gamma$, entonces $\pi: X \longrightarrow Y$ es un buen cociente categórico. 
Demostración. Demostremos la primera propiedad de la definición de cociente categórico, es decir, para todo $\mathrm{U} \subset \mathrm{Y}$ abierto

$$
\mathcal{O}_{Y}(\mathrm{U}) \simeq \mathcal{O}_{X}\left(\pi^{-1}(\mathrm{U})\right)^{\mathrm{G}} .
$$

Sea $U \in Y$, por $\operatorname{ser}\left\{V_{\gamma}\right\}$ una cubierta abierta de $Y$ tenemos que

$$
\mathrm{U}=\bigcup_{\alpha}\left(\mathrm{v}_{\alpha} \cap \mathrm{U}\right)
$$

para algunos índices $\alpha$, entonces $\mathrm{U} \cap \mathrm{V}_{\alpha} \subseteq \mathrm{V}_{\alpha}$ es abierto en $\mathrm{V}_{\alpha}$. Por hipótesis se tiene que

$$
\mathcal{O}_{\mathrm{V}_{\alpha}}\left(\mathrm{U} \cap \mathrm{V}_{\alpha}\right) \simeq \mathcal{O}_{\pi^{-1}\left(\mathrm{~V}_{\alpha}\right)}\left(\pi^{-1}\left(\mathrm{U} \cap \mathrm{V}_{\alpha}\right)\right)^{\mathrm{G}}
$$

para toda $\alpha$. Si $s \in \mathcal{O}_{Y}(\mathrm{U})$, entonces es regular en $\mathrm{V}_{\alpha} \cap \mathrm{U}$, es decir

$$
s \in \mathcal{O}_{\mathrm{V}_{\alpha}}\left(\mathrm{U} \cap \mathrm{V}_{\alpha}\right) \simeq \mathcal{O}_{\pi^{-1}\left(\mathrm{~V}_{\alpha}\right)}\left(\pi^{-1}\left(\mathrm{U} \cap \mathrm{V}_{\alpha}\right)\right)^{\mathrm{G}} \text {. }
$$

Denotemos por $s_{\mid \alpha}=s_{\mid U \cap V_{\alpha}}$. Para poder definir una sección en $\mathcal{O}_{X}\left(\pi^{-1}(U)\right)^{G}$ mediante $s$, es necesario verificar en las intersecciones de $\left(U \cap V_{\alpha}\right) \cap\left(U \cap V_{\beta}\right)$ las secciones obtenidas de $s$ vía las restricciones a los abiertos correspondientes son iguales. Para ello denotemos por $\mathrm{V}_{\alpha \beta}=\mathrm{U} \cap \mathrm{V}_{\alpha} \cap \mathrm{V}_{\beta}$, demostremos que

$$
\left(s_{\alpha}\right)_{\alpha \beta}=\left(s_{\mid u \cap v_{\alpha}}\right)_{\mid v_{\alpha \beta}} \quad \text { y } \quad\left(s_{\beta}\right)_{\alpha \beta}=\left(s_{\mid u \cap v_{\beta}}\right)_{\mid V_{\alpha \beta}}
$$

son iguales.

Observe que $V_{\alpha \beta}$ es un abierto de $U \cap V_{\alpha}$ y consideremos $\pi_{\alpha}=\pi_{\mid} u \cap V_{\alpha}$, por lo tanto

$$
\pi_{\alpha \mid \pi^{-1}\left(\mathrm{~V}_{\alpha \beta}\right)}: \pi_{\alpha}^{-1}\left(\mathrm{~V}_{\alpha \beta}\right) \longrightarrow \mathrm{V}_{\alpha \beta}
$$

es un buen cociente categórico. De manera análoga se tiene que

$$
\pi_{\beta \mid \pi^{-1}\left(\mathrm{~V}_{\alpha \beta}\right)}: \pi_{\beta}^{-1}\left(\mathrm{~V}_{\alpha \beta}\right) \longrightarrow \mathrm{V}_{\alpha \beta}
$$

es un buen cociente categórico.

Dado que $\pi_{\alpha}$ y $\pi_{\beta}$ son las restricciones de $\pi$ a los abiertos correspondientes, se tiene que $\pi_{\alpha}^{-1}\left(\mathrm{~V}_{\alpha \beta}\right)=\pi_{\beta}^{-1}\left(\mathrm{~V}_{\alpha \beta}\right)$. Ahora bien, el buen cociente categórico es único por lo tanto

$$
\mathcal{O}_{\mathrm{u} \cap \mathrm{V}_{\alpha}}\left(\mathrm{V}_{\alpha \beta}\right) \simeq \mathcal{O}_{\mathrm{u} \cap \mathrm{V}_{\beta}}\left(\mathrm{V}_{\alpha \beta}\right)
$$

de aquí que las secciones en las intersecciones coinciden. Lo cual implica

$$
\mathcal{O}_{\pi^{-1}\left(U \cap v_{\alpha}\right)}\left(\pi^{-1}\left(V_{\alpha \beta}\right)\right)^{G} \simeq \mathcal{O}_{\pi^{-1}\left(u \cap v_{\beta}\right)}\left(\pi^{-1}\left(V_{\alpha \beta}\right)\right)^{G}
$$

Es decir, dada una sección $s \in \mathcal{O}_{Y}(U)$ hemos construido una colección de secciones $\left\{\mathrm{s}_{\alpha}\right\}$, la restricción a cada abierto $\mathrm{U} \cap \mathrm{V}_{\alpha}$ que en las intersecciones de cualquiera dos de ellos, $V_{\alpha \beta}$, coinciden, entonces podemos definir una colección de secciones $\left\{\varphi_{\alpha}\left(s_{\alpha}\right)\right\}$, donde

$$
\varphi_{\alpha}: \mathcal{O}_{\mathrm{u} \cap \mathrm{v}_{\alpha}}\left(\mathrm{U} \cap \mathrm{v}_{\alpha}\right) \longrightarrow \mathcal{O}_{\pi^{-1}\left(\mathrm{U} \cap \mathrm{v}_{\alpha}\right)}\left(\pi^{-1}\left(\mathrm{U} \cap \mathrm{V}_{\alpha}\right)\right)^{\mathrm{G}}
$$

es un isomorfismo definido por ser $\pi$ un buen cociente categórico localmente. De aquí que $\left\{\varphi_{\alpha}\left(s_{\alpha}\right)\right\}$ generan una única sección global en $\pi-1(\mathrm{U}), \varphi(s) \in$ $\mathcal{O}_{X}(\pi-1(\mathrm{U}))$, dado $\varphi_{\alpha}\left(s_{\alpha}\right)$ es G-invariante para toda $\alpha$ se concluye que $\varphi s \in$ 
$\mathcal{O}_{X}\left(\pi^{-1}(\mathrm{U})\right)^{\mathrm{G}}$. Debido a que $\varphi_{\alpha}$ es un isomorfismo para toda $\alpha$, en realidad se tiene que

$$
\mathcal{O}_{Y}(\mathrm{U}) \simeq \mathcal{O}_{X}\left(\pi^{-1}(\mathrm{U})\right)^{\mathrm{G}} .
$$

Para la segunda propiedad de buen cociente categórico, sea $W$ un cerrado Ginvariante, entonces $\mathrm{U}=X \backslash W$ es un abierto G-invariante. Dado que $\pi$ es constante en órbitas, $Y=\pi(W) \cup \pi(\mathrm{U})$ con $\pi(W) \cap \pi(\mathrm{U})=\emptyset$. De nuevo, por ser $\pi$ constante en órbitas, se tiene que $U=\pi^{-1}(\pi(U))$, es decir, $\pi(U)$ es abierto, ya que $\pi$ es continua, por lo tanto, $\pi(W)$ es cerrado.

La tercera propiedad se deriva de la segunda, si $W, V$ con cerrados $G$-invariantes disjuntos, también lo son las imágenes bajo $\pi$.

Para finalizar este capítulo citaremos un teorema que nos indica cúando un anillo de elementos G-invariantes es finitamente generado. La demostración podrá consultarse en el libro de Dolgachev [4] o en el libro de Ferrer y Rittatore [5].

Teorema 2.29. Sea $\mathrm{G}$ un grupo reductivo que actúa en una variedad algebraica $\mathrm{X}$. Entonces

(a). $k[\mathrm{X}]^{\mathrm{G}}$ es finitamente generada como k-álgebra.

(b). El morfismo $\pi: \mathrm{X} \longrightarrow$ Specm $\mathrm{k}[\mathrm{X}]^{\mathrm{G}}$ inducido por $\mathrm{k}[\mathrm{X}]^{\mathrm{G}} \subseteq \mathrm{k}[\mathrm{X}]$ es un buen cociente categórico. 



\section{Capítulo 3}

\section{Variedades tóricas como cocientes}

\subsection{Preliminares}

El capítulo tiene por objetivo la aplicación de la teoría de invariantes a un ejemplo muy concreto, las variedades tóricas. En la sección 3.2 se dará la demostración de D. Cox [2] que caracteriza a cierto tipo de variedades tóricas. Iniciaremos recordando las definiciones y propiedades necesarias para ello en la sección 3.1, usando como referencias básicas [1] y [12]. En la sección 3.1 se trabajará con un campo algebraicamente cerrado arbitrario de característica cero. Sin embargo en la sección 3.2 se realizará el análisis sobre el campo de los números complejos. Los resultados principales están en la sección 3.2 con demostraciones completas.

Existen varias equivalencias para definir una variedad tórica, nos centraremos en dos únicamente. Para ello usaremos la definición de un toro algebraico (1.3) que se dio en el capítulo 1 .

Definición 3.1. Una variedad tórica es una variedad irreducible, $V$, la cual contiene a un toro $\mathrm{T}$ como un abierto tal que la acción de $\mathrm{T}$ en sí mismo se extiende a toda la variedad $V$, es decir, existe un morfimo $\varphi$ tal que

$$
\varphi: \mathrm{T} \times \mathrm{V} \longrightarrow \mathrm{V}
$$

es una acción algebraica.

Para la siguiente definición de variedades tóricas, requerimos de la definición de retícula.

Definición 3.2. Una retícula $\mathrm{N}$ es un grupo abeliano libre de rango finito. Es decir, si el rango de la retícula es $n$, se tiene que $N \simeq \mathbb{Z}^{n}$

Ahora bien, para el toro, $\mathrm{T}$ definimos los siguientes grupos.

Definición 3.3. Sea $\mathrm{T}$ un toro, se define

(a). Un subgrupo a un parámetro de T es un morfismo $\lambda: k^{*} \longrightarrow$ T el cual es un homomorfismo de grupos. El conjunto de subgrupos uniparamétricos $\mathrm{Y}(\mathrm{T})=\operatorname{Hom}\left(\mathrm{k}^{*}, \mathrm{~T}\right)$ es un grupo.

(b). Un caracter de un $\mathrm{T}$ es un morfismo $\chi: T \longrightarrow \mathrm{k}^{*}$ el cual es un homomorfismo de grupo. El conjunto de caracteres $X(T)=\operatorname{Hom}\left(T, k^{*}\right)$ es un grupo.

Observación 3.4. Cuando $T=\left(k^{*}\right)^{n}$ se tienen las siguientes observaciones. 
- Sea $u=\left(b_{1}, \cdots, b_{n}\right) \in \mathbb{Z}^{n}$ define un subgrupo a un parámetro $\lambda^{\mathfrak{u}}$ : $\mathrm{k}^{*} \longrightarrow \mathrm{T}$,

$$
\lambda^{u}(t)=\left(t^{b_{1}}, \cdots, t^{b_{n}}\right) .
$$

Todos los subgrupos a un párametro de $\mathrm{T}=\left(\mathrm{k}^{*}\right)^{\mathrm{n}}$ tienen esta forma. Por lo que son un grupo, $N$, isomorfo a $\mathbb{Z}^{n}$.

- Sea $m=\left(a_{1}, \cdots, a_{n}\right) \in \mathbb{Z}^{n}$ definimos el caracter $\chi^{m}: T \longrightarrow k^{*}$ por

$$
\chi^{m}\left(t_{1}, \cdots, t_{n}\right)=t_{1}{ }^{a_{1}} \cdot t_{2}{ }^{a_{2}} \cdots t_{n}{ }^{a_{n}} .
$$

$\mathrm{Al}$ igual que los subgrupos a un parámetro, todos los caracteres de $\mathrm{T}=$ $\left(k^{*}\right)^{n}$ son de esta forma y por tanto forman un grupo, $M$, isomorfo a $\mathbb{Z}^{n}$.

Para el caso cuando $T \simeq\left(k^{*}\right)^{n}$ se tiene que tanto el grupo de caracteres como el de subgrupos a un parámetro son isomorfos a un grupo abeliano libre de rango finito, con rango igual a la dimensión del toro, $\mathrm{T}$.

Entre estos dos grupos se tiene un apareamiento perfecto.

Observación 3.5. Sean T, N y $M$ como en la observación anterior. Entonces, existe un apareamiento natural bilineal

$$
\langle,\rangle: \mathrm{M} \times \mathrm{N} \longrightarrow \mathbb{Z}
$$

definido por

- Dado un caracter $\chi^{m}$ y un subgrupo a un parámetro $\lambda^{u}$ la composición $\chi^{\mathrm{m}} \circ \lambda^{\mathfrak{u}}: k^{*} \longrightarrow k^{*}$ es un subgrupo a un parámetro de $k^{*}$, el cual está dado por $t \longrightarrow t^{l}$ con $l \in \mathbb{Z}$. Entonces definimos

$$
\langle\mathrm{m}, \mathrm{u}\rangle=\mathrm{l} \text {. }
$$

- En el caso concreto de que $T=\left(k^{*}\right)^{n}$ se tiene que

$$
\langle\mathrm{m}, \mathrm{n}\rangle=\sum_{i=1}^{n} a_{i} b_{i},
$$

$$
\text { con } \mathrm{m}=\left(\mathrm{b}_{1}, \cdots, \mathrm{b}_{\mathrm{n}}\right) \in \mathbb{Z}^{\mathrm{n}} \text { y } \mathrm{u}=\left(\mathrm{a}_{1}, \cdots, \mathrm{a}_{\mathrm{n}}\right) \in \mathbb{Z}^{\mathrm{n}} .
$$

Estos grupos abelianos libres de rango finito son las retículas asociadas al toro $\mathrm{T}$, ambas son duales una de la otra. Denotaremos por $\mathrm{T}_{\mathrm{N}}$ el toro asociado a la retícula $\mathrm{N}$ y por $\mathrm{M}$ a su retícula dual correspondiente.

Un semigrupo es un conjunto $S$ con una operación binaria asociativa y un elemento distinguido que denotaremos por identidad. Los semigrupos de nuestro interés son los afines, los cuales cumplen los siguiente.

Definición 3.6. Un semigrupo $S$ es un semigrupo afín si

- La operación binaria en $\mathrm{S}$ es conmutativa. En este caso denotaremos a la operación como la suma, + y el elemento identidad por 0 . Entonces un conjunto finito $S^{\prime}$ genera el semigrupo conmutativo

$$
\mathbb{N S}^{\prime}=\sum_{m \in S^{\prime}}\left\{a_{m} m \mid a_{m} \in \mathbb{N}\right\} \subseteq S
$$

- $S$ es finitamente generado si existe un conjunto finito $S^{\prime}$ tal que $\mathbb{N} S^{\prime}=S$. 
- El semigrupo $S$ puede ser encajado en una retícula $M$.

Los semigrupos afines permiten asociarles una k-álgebra de semigrupo, la cual es el espacio vectorial con base $\mathrm{k}$ y la multiplicación inducida por la estructura de grupo. Nos enfocaremos en el caso del toro. Sea $M$ su retícula de caracteres. Dado $\mathrm{m} \in M$ se tiene el caracter $\chi^{\mathrm{m}}$. Entonces definimos

$\mathrm{k}[\mathrm{S}]=\left\{\sum_{\mathrm{m}} \mathrm{c}_{\mathrm{m}} \chi^{\mathrm{m}} \mid \mathrm{c}_{\mathrm{m}} \in \mathrm{k} \quad\right.$ y $\quad \mathrm{c}_{\mathrm{m}}=0 \quad$ para casi toda $\mathrm{m}$ salvo un número finito $\}$ con la multiplicación inducida por

$$
x^{m} \cdot x^{m^{\prime}}=\chi^{m+m^{\prime}}
$$

Si $S=\mathbb{N} S^{\prime}$ para algún $S^{\prime}=\left\{m_{1}, \cdots, m_{n}\right\}$ entonces $k[S]=k\left[\chi^{m_{1}}, \cdots, \chi^{m_{n}}\right]$.

Con esto podemos citar la siguiente proposición. Ver [1, Prop. 1.1.14]

Proposición 3.7. Sea $\mathrm{S}$ un semigrupo afín. Entonces

(a). $\mathrm{k}[\mathrm{S}]$ es un dominio entero y finitamente generado como k-álgebra.

(b). $\operatorname{Specm}(k[S])$ es una variedad tórica cuyo toro tiene retícula de caracteres $\mathbb{Z} \mathrm{S}$.

La siguiente descripción de una variedad tórica nos permitirá fijar la notación que se usará en la siguiente sección.

Consideremos los espacios vectoriales $N_{\mathbb{R}}=\mathrm{N} \otimes_{\mathbb{Z}} \mathbb{R}$ y su dual $M_{\mathbb{R}}=M \otimes_{\mathbb{Z}} \mathbb{R}$ (ambos espacios son isomorfos a $\mathbb{R}^{n}$ ). El apareamiento entre las retículas $\mathrm{N}$ y $\mathrm{M}$ induce un apareamiento natural entre estos espacios vectoriales

$$
\begin{aligned}
\langle,\rangle: M_{\mathbb{R}} \times N_{\mathbb{R}} & \longrightarrow \mathbb{R} \\
\left\langle\chi^{\mathrm{m}} \otimes \mathrm{r}_{1}, \lambda^{\mathrm{u}} \otimes \mathrm{r}_{2}\right\rangle & \longrightarrow\langle\mathrm{m}, \mathrm{u}\rangle \mathrm{r}_{1} \mathrm{r}_{2},
\end{aligned}
$$

con $r_{1}, r_{2}$ números reales.

Definición 3.8. Un cono convexo poliédrico en $\mathrm{N}_{\mathbb{R}}$ es un conjunto de la forma

$$
\sigma=\operatorname{Cono}(S)=\left\{\sum_{\mathfrak{u} \in S} \lambda_{\mathfrak{u}} u \mid \lambda_{\mathfrak{u}} \geqslant 0\right\} \subseteq \mathrm{N}_{\mathbb{R}}
$$

donde $S \subseteq \mathrm{N}_{\mathbb{R}}$ es un conjunto finito. Se dice que $\sigma$ está generado por $S$. Por convención $\operatorname{Cono}(\emptyset)=0$

Definición 3.9. Sea $\sigma \subseteq \mathrm{N}_{\mathbb{R}}$ un cono poliédrico convexo, se define su cono dual como

$$
\sigma^{\vee}=\left\{m \in M_{\mathbb{R}} \mid\langle m, u\rangle \geqslant 0 \text { para toda } u \in \sigma\right\} .
$$

Dado $m \neq 0$ en $M_{\mathbb{R}}$ se tiene el hiperplano

$$
\mathrm{H}_{\mathrm{m}}=\left\{\mathrm{u} \in \mathrm{N}_{\mathbb{R}} \mid\langle\mathrm{m}, \mathrm{u}\rangle=0\right\} \subseteq \mathrm{N}_{\mathbb{R}}
$$

y el semi plano cerrado

$$
\mathrm{H}_{\mathrm{m}}{ }^{+}=\left\{\mathrm{u} \in \mathrm{N}_{\mathbb{R}} \mid\langle\mathrm{m}, \mathrm{u}\rangle \geqslant 0\right\} \subseteq \mathrm{N}_{\mathbb{R}} .
$$


Definición 3.10. Una cara de un cono $\sigma$ es un conjunto de la forma $\tau=H_{m} \cap \sigma$ para algún $m \in \sigma^{\vee}$. La notación para una cara es $\tau \preceq \sigma$. Observe que si $m=0$ se obtiene que $\sigma$ es cara de sí mismo. Las caras $\tau \neq \sigma$ se llaman caras propias y se denotarán por $\tau \prec \sigma$.

Definición 3.11. Dada una cara $\tau \preceq \sigma$ se define

$$
\begin{aligned}
\tau^{\perp} & =\left\{m \in M_{\mathbb{R}} \mid\langle m, u\rangle=0 \quad \text { para toda } u \in \tau\right\} \\
\tau^{*} & =\left\{m \in \sigma^{\vee} \mid\langle m, u\rangle=0 \quad \text { para toda } u \in \tau\right\} \\
& =\sigma^{\vee} \cap \tau^{\perp} .
\end{aligned}
$$

La anterior observación establece una relación entre las caras de un cono $\sigma \subseteq$ $\mathrm{N}_{\mathbb{R}}$ y las caras de su cono dual $\sigma^{\vee} \subseteq M_{\mathbb{R}}$.

Definición 3.12. Sea $\sigma \subseteq \mathrm{N}_{\mathbb{R}}$.

- $\sigma$ es un cono fuertemente convexo si $(\sigma) \cap(-\sigma)=0$.

- $\sigma$ es un cono racional si $\sigma=\operatorname{Cono}(S)$ para algún conjunto finito $S \subseteq N$.

Los conos que nos serán de utilidad son los conos poliédricos racionales fuertemente convexos. Por lo que de aquí en adelante se entenderá por cono $\sigma$ un cono poliédrico racional fuertemente convexo.

Sea $\sigma$ un cono, y $\rho$ una cara de dimensión 1, es decir, un rayo. Dado que $\sigma$ es racional también lo es $\rho$, por lo tanto el semigrupo $\rho \cap N$ está generado por un único elemento mínimo, $\mathfrak{u}_{\rho} \in \rho \cap \mathrm{N}$. Como consecuencia se tiene que $\sigma$ está generado por sus rayos generadores de sus caras de dimensión 1.

Definición 3.13. Un cono $\sigma$ es simplicial si sus generadores mínimos son linealmente independientes sobre $\mathbb{R}$

Para relacionar estas definiciones con las variedades tóricas se requiere de la siguiente observación y proposición.

Observación 3.14. Sea $\sigma \subseteq \mathrm{N}_{\mathbb{R}}$, considere los siguientes puntos reticulares

$$
S_{\sigma}=\sigma^{\vee} \cap M \subseteq M
$$

los cuales forman un semigrupo.

Para cada $\tau \preceq \sigma$ su semigrupo asociado está dado por

$$
\mathrm{S}_{\tau}=\mathrm{S}_{\sigma}+\mathbb{Z}(-\mathrm{m}) \text {. }
$$

Proposición 3.15 (Lema de Gordan). $\mathrm{S}_{\sigma}$ es finitamente generado.

Ver, por ejemplo, [1, Prop. 1.2.17].

Es decir $S_{\sigma}$ es un semigrupo afín (ver 3.6). Entonces por la proposición 3.7, $S_{\sigma}$ tiene asociada la $k$-álgebra de semigrupo $k\left[S_{\sigma}\right]$ de tipo finito, . Ahora bien, a una cara $\tau \preceq \sigma$ se tiene que $k\left[S_{\tau}\right]=k\left[S_{\sigma}\right]_{\chi^{m}}$. Por lo tanto se tiene el siguiente teorema que conecta una variedad tórica afín con un cono, ver, por ejemplo, [1, Teo. 1.2.18]. 
Teorema 3.16. Sea $\sigma \subseteq \mathrm{N}_{\mathbb{R}}$ con semigrupo $S_{\sigma}$, entonces

$$
\mathrm{u}_{\sigma}=\operatorname{Specm}\left(\mathrm{k}\left[\mathrm{S}_{\sigma}\right]\right)
$$

es una variedad tórica afín.

Hemos construido una variedad tórica afín a partir de un cono, ahora daremos una construcción de una variedad tórica a partir de una colección finita de conos.

Definición 3.17. Un abanico $\Sigma \subseteq \mathrm{N}_{\mathbb{R}}$ es una colección finita de conos, tales que

(a). Todo cono $\sigma \in \Sigma$ es un cono fuertemente convexo y racional.

(b). Para todo $\sigma \in \Sigma$ cada cara de $\sigma$ pertenece a $\Sigma$.

(c). Para cualesquiera $\sigma_{1}, \sigma_{2}$ en $\Sigma$, la intersección $\sigma_{1} \cap \sigma_{2}$ es una cara de ambos.

(d). El soporte de $\Sigma$ es $|\Sigma|=\cup_{\sigma \in \Sigma} \sigma \subseteq \mathrm{N}_{\mathbb{R}}$.

(e). $\Sigma(r)$ es el conjunto de conos de dimensión $r$.

Al igual que en los conos podemos definir lo que es un abanico simplicial. Se dice que $\Sigma$ es simplicial si todo cono $\sigma \in \Sigma$ es simplicial.

Observación 3.18. Dado un abanico $\Sigma$, podemos construir una variedad tórica asociada a $\Sigma$, pegando las variedades tóricas $U_{\sigma}$ por medio de las caras provenientes de interseción de dos conos. Denotaremos por $\mathrm{X}_{\Sigma}$ a dicha variedad tórica. Consultar [1, Sección 3.1].

Ahora, dado que $X_{\Sigma}$ contiene un toro $T_{N}$ el cual actúa en la variedad, podemos preguntarnos sobre las $\mathrm{T}_{\mathrm{N}}$-órbitas. Dichas órbitas están caracterizadas por el siguiente teorema. La demostración se puede consultar en [1, Teo. 3.2.6].

Teorema 3.19 (Correspondencia órbita-cono). Sea $\mathrm{X}_{\Sigma}$ la variedad tórica asociada al abanico $\Sigma$. Entonces

(a). Existe una correspondencia biyectiva

$$
\begin{aligned}
\{\text { conos } \sigma \in \Sigma\} & \longleftrightarrow\left\{\mathrm{T}_{\mathrm{N}} \text {-órbitas en } \mathrm{X}_{\Sigma}\right\} \\
\sigma & \longleftrightarrow \text { órb }_{\mathrm{T}_{\mathrm{N}}}=\operatorname{Hom}_{\mathbb{Z}}\left(\sigma^{\perp} \cap M, \mathrm{k}^{*}\right)
\end{aligned}
$$

(b). Sea $n=\operatorname{dim} \mathbb{N}_{\mathbb{R}}$. Para cada cono $\sigma \in \Sigma$, dimórb $\operatorname{T}_{\mathrm{N}}(\sigma)=\mathfrak{n}-\operatorname{dim} \sigma$.

(c). El abierto afín $\mathrm{U}_{\sigma}$ es la unión de sus órbitas

$$
\mathrm{u}_{\sigma}=\bigcup_{\tau \preceq \sigma} \operatorname{orb}_{\mathrm{T}_{\mathrm{N}}}(\tau)
$$

(d). $\tau \preceq \sigma$ si y sólo si órb $\mathrm{T}_{\mathrm{N}}(\sigma) \subseteq \overline{\operatorname{orb}_{\mathrm{T}_{\mathrm{N}}}}(\tau)$ y

$$
\overline{\operatorname{orb}_{\mathrm{T}_{N}}(\tau)}=\bigcup_{\tau \preceq \sigma} \operatorname{orb}_{\mathrm{T}_{\mathrm{N}}}(\sigma),
$$

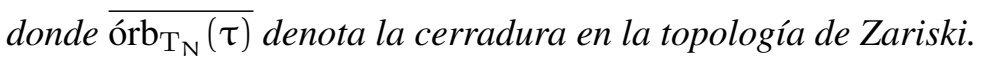

A continuación definiremos un tipo especial de morfismos entre variedades tóricas relacionados con los abanicos asociados a ellas. 
Definición 3.20. Sean $N_{1}, N_{2}$ dos retículas con $\Sigma_{1}$ un abanico en $\left(N_{1}\right)_{\mathbb{R}}$ y $\Sigma_{2}$ un abanico en $\left(N_{2}\right)_{\mathbb{R}}$. Una aplicación $\mathbb{Z}$-lineal $\bar{\phi}: \mathrm{N}_{1} \longrightarrow \mathrm{N}_{2}$ es compatible con los abanicos $\Sigma_{1}$ y $\Sigma_{2}$ si para cada cono en $\sigma_{1} \in \Sigma_{1}$ existe un cono $\sigma_{2} \in \Sigma_{2}$ tal que $\bar{\phi}_{\mathbb{R}}\left(\sigma_{1}\right) \subseteq \sigma_{2}$.

Con ello podemos dar la siguiente definición.

Definición 3.21. Sean $X_{\Sigma_{1}}$ y $X_{\Sigma_{2}}$ dos variedades tóricas normales con abanicos asociados $\Sigma_{1}$ abanico en $\left(N_{1}\right)_{\mathbb{R}}$ y $\Sigma_{2}$ abanico en $\left(N_{2}\right)_{\mathbb{R}}$. Un morfismo $\phi: X_{\Sigma_{1}} \longrightarrow$ $X_{\Sigma_{2}}$ es tórico si $\phi$ envía el toro $\mathrm{T}_{\mathrm{N}_{1}} \subseteq \mathrm{X}_{\Sigma_{1}}$ en el toro $\mathrm{T}_{\mathrm{N}_{1}} \subseteq \mathrm{X}_{\Sigma_{2}}$ y $\phi_{\mid \mathrm{T}_{\mathrm{N}_{1}}}$ es un homomorfismo de grupos.

El siguiente teorema describe cómo se relacionan las aplicaciones compatibles entre abanicos y morfismos tóricos.

Teorema 3.22 (Ver [10]). Sean $\mathrm{N}_{1}, \mathrm{~N}_{2}$ dos retículas y sea $\Sigma_{i}$ un abanico en $\left(\mathrm{N}_{i}\right)_{\mathbb{R}}$, $i=1,2$.

(a). Si $\bar{\phi}: \mathrm{N}_{1} \longrightarrow \mathrm{N}_{2}$ es una aplicación $\mathbb{Z}$-lineal compatible con los abani$\cos \Sigma_{1}$ y $\Sigma_{2}$, entonces existe un morfismo tórico $\phi: \mathrm{X}_{\Sigma_{1}} \longrightarrow \mathrm{X}_{\Sigma_{2}}$ tal que $\phi_{\mid \mathrm{T}_{\mathrm{N}_{1}}}$ es la aplicación

$$
\bar{\phi} \otimes 1: \mathrm{N}_{1} \otimes_{\mathbb{Z}} \mathrm{k}^{*} \longrightarrow \mathrm{N}_{2} \otimes_{\mathbb{Z}} \mathrm{k}^{*}
$$

(b). Recíprocamente, si $\phi: \mathrm{X}_{\Sigma_{1}} \longrightarrow \mathrm{X}_{\Sigma_{2}}$ es un morfismo tórico, entonces $\phi$ induce una aplicación $\mathbb{Z}$-lineal $\bar{\phi}: \mathrm{N}_{1} \longrightarrow \mathrm{N}_{2}$ que es compatible con los abanicos $\Sigma_{1}$ y $\Sigma_{2}$.

\subsection{Variedades tóricas como cocientes}

Esta sección tiene por objetivo dar la demostración de que toda variedad tórica es un casi buen cociente de una variedad algebraica afín por la acción de un grupo algebraico adecuado. Nos guiaremos de la sección 5.1 del libro [1] y del artículo original [2].

Iniciaremos con el caso cuando la variedad tórica no tiene un factor toro, los primeros resultados serán bajo esta conidición a menos que se indique lo contrario.

Proposición 3.23. Sea $X_{\Sigma}$ la variedad tórica asociada al abanico $\Sigma$. Son Equivalentes

(a). $X_{\Sigma}$ tiene un factor toro

(b). Existe un morfismo no constante $\mathrm{X}_{\Sigma} \longrightarrow \mathrm{k}^{*}$

(c). $\mathrm{u}_{\mathrm{p}}, \mathrm{p} \in \Sigma(1)$ no genera a $\mathrm{N}_{\mathbb{R}}$

Demostración. (a). Supongamos que $X_{\Sigma}$ tiene un factor toro, es decir $X_{\Sigma} \simeq X_{\Sigma^{\prime}} \times\left(k^{*}\right)^{r}$ para $r>0$ y para alguna variedad tórica $X_{\Sigma^{\prime}}$. Sea $\lambda$ un caracter no trivial de $\left(k^{*}\right)^{r}$, entonces esto define un morfismo no constante

$$
X_{\Sigma} \simeq X_{\Sigma^{\prime}} \times\left(k^{*}\right)^{r} \longrightarrow\left(k^{*}\right)^{r} \longrightarrow k^{*}
$$

donde el primer morfismo es la proyección en la segunda entrada y el segundo es el carácter $\lambda$. 
(b). Sea $M$ y $N$ las retículas asociadas al toro $T_{N}$. Recordemos que $k[M]$ se descompone como suma directa de $\chi^{m}$, caracteres del toro, $\chi^{m}: T_{N} \longrightarrow$ $k^{*}$. Por lo tanto todo morfismo $T_{N} \longrightarrow k^{*}$ corresponde a los elementos invertibles en el anillo de coordenadas de $T_{N}$, es decir en $k[M]$. Luego, dichos morfismos son de la forma $c \chi^{m}$ con $c \in k^{*}$. Entonces, cada morfismo no constante $\phi: X_{\Sigma} \longrightarrow k^{*}$ cumple que $\phi_{\mid \mathrm{T}_{\mathrm{N}}}=\chi^{\mathrm{m}}$ con $\mathrm{m} \in M \backslash 0$. Entonces $\phi$ es un morfismo tórico que proviene de un homomorfismo entre retículas (no trivial) $\bar{\phi}: \mathrm{N} \longrightarrow \mathbb{Z}$. Dado que $k^{*}$ proviene del abanico trivial entonces $\bar{\phi}_{\mathbb{R}}$ envía todos los conos de $\Sigma$ al origen. Lo cual implica que $u_{\rho} \in \operatorname{ker} \bar{\phi}_{\mathbb{R}}$ para todo $\rho \in \Sigma(1)$, por lo tanto $u_{\rho}$ no genera todo $\mathrm{N}_{\mathbb{R}}$.

(c). Supongamos que $u_{\rho}, \rho \in \Sigma(1)$ no genera todo $N$. Sea $N^{\prime}$ la retícula generada por $\left(u_{\rho} \mid \rho \in \Sigma(1)\right) \cap N$ la cual es una subretícula propia de $N$. Luego $\mathrm{N} / \mathrm{N}^{\prime}$ es libre de torsión, ya que la retícula es saturada, es decir, sea $a \in \mathbb{Z}$ entonces $a n^{\prime} \in N^{\prime}$ si y sólo si $n \in N^{\prime}$, esto se debe a que la variedad tórica asociada a $\mathrm{N}^{\prime}$ es un toro, por lo que existe $\mathrm{N}^{\prime \prime}$ tal que $\mathrm{N}=\mathrm{N}^{\prime} \times \mathrm{N}^{\prime \prime}$, por lo que $\Sigma$ lo podemos considerar como un abanico $\Sigma^{\prime}$ en $N_{\mathbb{R}}^{\prime}$, entonces $\Sigma$ es el producto de abanicos $\Sigma=\Sigma^{\prime} \times \Sigma^{\prime \prime}$ con $\Sigma^{\prime \prime}$ el abanico trivial en $N_{\mathbb{R}}^{\prime \prime}$. Luego, tenemos el siguiente isomorfismo

$$
\mathrm{X}_{\Sigma} \simeq \mathrm{X}_{\Sigma^{\prime}} \times \mathrm{T}_{\mathrm{N}^{\prime \prime}} \simeq \mathrm{X}_{\Sigma^{\prime}} \times\left(\mathrm{k}^{*}\right)^{\mathrm{n}-\mathrm{s}}
$$

con $\operatorname{dim} \mathrm{N}_{\mathbb{R}}=\mathrm{n} \mathrm{y} \operatorname{dim} \mathrm{N}_{\mathbb{R}}^{\prime}=\mathrm{s}$.

De este resultado obtenemos la siguiente proposición. Ver [1, Prop. 4.1.3].

Proposición 3.24. Se tiene la siguiente sucesión exacta

$$
0 \longrightarrow \mathrm{M} \longrightarrow \operatorname{Div}_{\mathrm{T}_{\mathrm{N}}}\left(\mathrm{X}_{\Sigma}\right) \longrightarrow \mathrm{Cl}\left(\mathrm{X}_{\Sigma}\right) \longrightarrow 0
$$

donde la primera aplicación envía a $\mathrm{m}$ a div $\chi^{\mathrm{m}}$ en tanto que la segunda envía un divisor $\mathrm{T}_{\mathrm{N}}$-invariante en su representante en $\mathrm{Cl}\left(\mathrm{X}_{\Sigma}\right)$. Dicha sucesión es exacta corta si y sólo si

$$
\left\{u_{p} \mid p \in \Sigma(1)\right\}
$$

genera a todo $\mathrm{N}_{\mathbb{R}}$, es decir, si y sólo si $\mathrm{X}_{\Sigma}$ no tiene un factor toro.

Demostración. Observe que para todo $\mathrm{D} \in \operatorname{Div}_{\mathrm{T}_{\mathrm{N}}}\left(\mathrm{X}_{\Sigma}\right)$, se tiene que

$$
\text { órb }(0) \cap \mathrm{D}=\emptyset
$$

por las condiciones de invarianza. Por otro lado,

$$
\operatorname{Div}_{T_{N}}\left(X_{\Sigma}\right)=\bigoplus_{\rho} \mathbb{Z} D_{\rho}
$$

en donde $\mathrm{D}_{\rho}=\overline{\operatorname{orb}(\rho)}$ con $\rho \in \Sigma(1)$, esta igualdad se debe al teorema de correspondecia de conos y órbitas $\mathrm{T}_{\mathrm{N}}$-invariantes y ejemplo 2.3 de [10]. De hecho, $\mathrm{D}_{\rho}$ con $\rho \in \Sigma(1)$ son una base para $\operatorname{Div}_{T_{N}}\left(X_{\Sigma}\right)$ por condiciones de irreducibilidad. 
Entonces $D_{\rho}$ son las componentes irreducibles de $X_{\Sigma} \backslash T_{N}$ y podemos aplicar el teorema 4.0.20 del libro [1] y tenemos la siguiente sucesión exacta

$$
\operatorname{Div}_{\mathrm{T}_{\mathrm{N}}}\left(\mathrm{X}_{\Sigma}\right) \longrightarrow \mathrm{Cl}\left(\mathrm{X}_{\Sigma}\right) \longrightarrow \mathrm{Cl}\left(\mathrm{T}_{\mathrm{N}}\right) \longrightarrow 0
$$

Sin embargo, por el teorema 4.0.18 de [1] se tiene que $\operatorname{Cl}\left(\mathrm{T}_{\mathrm{N}}\right)=0$, dado que $k\left[T_{N}\right]$ es un DFU. Por lo que todo divisor es un divisor principal. Entonces, de la sucesión (1), obtenemos

$$
\operatorname{Div}_{\mathrm{T}_{\mathrm{N}}}\left(\mathrm{X}_{\Sigma}\right) \longrightarrow \mathrm{Cl}\left(\mathrm{X}_{\Sigma}\right) \longrightarrow 0
$$

por lo tanto $\operatorname{Div}_{T_{N}}\left(X_{\Sigma}\right) \longrightarrow \mathrm{Cl}\left(X_{\Sigma}\right)$ es suprayectiva.

Ahora bien, sea $\mathrm{m} \in M$ y considere $\operatorname{div}\left(\chi^{\mathrm{m}}\right)$, este divisor es $\mathrm{T}_{\mathrm{N}}$-invariante por proposición 2.7 de [10], por lo tanto la composición $m \rightarrow \operatorname{div}\left(\chi^{m}\right)$ es cero. Ahora sea $D \in \operatorname{Div}_{T_{N}}\left(X_{\Sigma}\right)$ tal que $D$ va a dar a cero en $\operatorname{Cl}\left(X_{\Sigma}\right)$, entonces existe $f \in$ $k\left(X_{\Sigma}\right)^{*}$ tal que $D=\operatorname{div}(f)$. Por $(1) \operatorname{div}(f)$ es principal en $T_{N}$ por lo que existe $f^{\prime}$, con $\operatorname{div}(f)_{\mid T_{N}}=\operatorname{div}\left(f^{\prime}\right)$. Pero todo divisor principal es de Cartier, entonces por proposición 2.10 de [10] existe $m \in M$

$$
\operatorname{div}\left(f^{\prime}\right)=\operatorname{div}\left(\chi^{m}\right)=\sum_{\rho \in \Sigma(1)}\left\langle m, u_{\rho}\right\rangle D_{\rho}
$$

Probando así la exactitud de la sucesión

$$
M \longrightarrow \operatorname{Div}_{\mathrm{T}_{\mathrm{N}}}\left(\mathrm{X}_{\Sigma}\right) \longrightarrow \mathrm{Cl}\left(\mathrm{X}_{\Sigma}\right) \longrightarrow 0
$$

Solo resta demostrar que (3) es una sucesión exacta corta. Supongamos que $m \in$ $M$ con $\operatorname{div}\left(\chi^{m}\right)=0$, entonces $\left\langle m, u_{\rho}\right\rangle=0$ para todo $u_{\rho} \in \Sigma(1)$, entonces si $\left\{u_{\rho} \mid \rho \in \Sigma(1)\right\}$ genera todo $N_{\mathbb{R}}$ se tiene que $m=0$. Por último supongamos que (3) es exacta corta y que $\left\{u_{\rho} \mid \rho \in \Sigma(1)\right\}$ generan $N_{\mathbb{R}}^{\prime} \subset N_{\mathbb{R}}$, podemos suponer que difieren de un solo generador, digamos $u_{\tau}$ (ya que $N_{\mathbb{R}}$ es de rango finito). Considere $\Sigma^{\prime}$ el abanico generado por $\left\{u_{\rho} \mid \rho \in \Sigma(1)\right\} \cup\left\{u_{\tau}\right\}$, el cual por construcción genera a todo $N_{\mathbb{R}}$. Sea $X_{\Sigma^{\prime}}$ la variedad asociada al abanico $\Sigma^{\prime}$, por construcción $X_{\Sigma}$ es un abierto en $X_{\Sigma}$ puesto que es unión de los abiertos $U_{\sigma}$ con $\sigma \in \Sigma \subset \Sigma^{\prime}$. Por las condiciones de invarianza de las órbitas asociadas a los conos de dimensión uno, tenemos que el divisor $D_{\tau}$ es la componente irreducible de $X_{\Sigma^{\prime}} \backslash X_{\Sigma}$. De nuevo por la proposición 4.0.20 de [1] podemos considerar la siguiente sucesión exacta corta,

$$
\mathrm{M} \longrightarrow \operatorname{Div}_{\mathrm{T}_{\mathrm{N}}}\left(\mathrm{X}_{\Sigma^{\prime}}\right) \longrightarrow \mathrm{Cl}\left(\mathrm{X}_{\Sigma^{\prime}}\right) \longrightarrow \mathrm{Cl}\left(\mathrm{X}_{\Sigma}\right) \longrightarrow 0
$$

Por la exactitud de (4) existe $m \neq 0 \in M$ con caracter asociado al divisor

$$
\operatorname{div}\left(\chi^{m}\right)=\left\langle m, u_{\tau}\right\rangle D_{\tau} .
$$

Por otro lado $\operatorname{div}\left(\chi^{m}\right)_{\mid X_{\Sigma}}=0$ puesto que su soporte no contiene a ningún $D_{\rho}$ con $\rho \in \Sigma(1)$. Mas aún, $\operatorname{div}\left(\chi^{m}\right)_{\mid X_{\Sigma}}$ es un divisor principal en $X_{\Sigma}$ y $T_{N}$-invariante. Por lo que hemos contruido un caracter, $\chi^{\mathrm{m}}$ diferente de cero tal que $\operatorname{div}\left(\chi^{\mathrm{m}}\right)=$ $\operatorname{div}(0)$ en $X_{\Sigma}$ contradiciendo la sucesión exacta corta con la que iniciamos. Por lo tanto $\left\{u_{\rho} \mid \rho \in \Sigma(1)\right\}$ genera a todo $N_{\mathbb{R}}$. 
Dado que

$$
\operatorname{Div}_{T_{N}}\left(X_{\Sigma}\right)=\bigoplus_{p} \mathbb{Z} D_{p}
$$

Es decir tenemos la siguiente sucesión exacta corta

$$
0 \longrightarrow \mathrm{M} \longrightarrow \bigoplus_{\mathrm{p}} \mathbb{Z} \mathrm{D}_{\mathrm{p}} \longrightarrow \mathrm{Cl}\left(\mathrm{X}_{\Sigma}\right) \longrightarrow 0
$$

o bien

$$
0 \longrightarrow \mathrm{M} \longrightarrow \mathbb{Z}^{\Sigma(1)} \longrightarrow \mathrm{Cl}\left(\mathrm{X}_{\Sigma}\right) \longrightarrow 0,
$$

ya que para cada $m \in M$ se envía a $\operatorname{div}\left(\chi^{m}\right)=\Sigma_{p}\left\langle m, u_{p}\right\rangle D_{p}$.

Aplicando el funtor $\operatorname{Hom}_{\mathbb{Z}}\left(-, k^{*}\right)$ a esta ultima sucesión exacta corta tenemos

$$
1 \longrightarrow \operatorname{Hom}_{\mathbb{Z}}\left(\mathrm{Cl}\left(\mathrm{X}_{\Sigma}\right), \mathrm{k}^{*}\right) \longrightarrow \operatorname{Hom}_{\mathbb{Z}}\left(\mathbb{Z}^{\Sigma(1)}, \mathrm{k}^{*}\right) \longrightarrow \operatorname{Hom}_{\mathbb{Z}}\left(M, k^{*}\right) \longrightarrow 1
$$

Recordemos que tenemos los siguientes isomorfismos

$$
\begin{aligned}
\operatorname{Hom}_{\mathbb{Z}}\left(\mathbb{Z}^{\Sigma(1)}, k^{*}\right) & \simeq\left(k^{*}\right)^{\Sigma(1)} \\
\operatorname{Hom}_{\mathbb{Z}}\left(M, k^{*}\right) & \simeq \mathrm{T}_{\mathrm{N}},
\end{aligned}
$$

y definimos el siguiente grupo

$$
\mathrm{G}=\operatorname{Hom}_{\mathbb{Z}}\left(\mathrm{Cl}\left(\mathrm{X}_{\Sigma}\right), \mathrm{k}^{*}\right) .
$$

Por lo que sucesión exacta corta se puede escribir como

$$
1 \longrightarrow \mathrm{G} \longrightarrow\left(\mathrm{k}^{*}\right)^{\Sigma(1)} \longrightarrow \mathrm{T}_{\mathrm{N}} \longrightarrow 1
$$

Una propiedad del grupo $\mathrm{G}$ es la siguiente

Lema 3.25. Sea $\mathrm{G} \subseteq\left(\mathrm{k}^{*}\right)^{\Sigma(1)}$ el grupo definido previamente. Entonces se cumple

(a). $\mathrm{Cl}\left(\mathrm{X}_{\Sigma}\right)$ es el grupo de caracteres de $\mathrm{G}$.

(b). G es el producto de un toro y un grupo finito abeliano. En particular G es reductivo.

(c). Dada una base $e_{1}, \cdots, e_{\mathrm{n}}$ de $M$ tenemos

$$
\begin{aligned}
\mathrm{G} & =\left\{\left(\mathrm{t}_{\mathrm{p}}\right) \in\left(\mathrm{k}^{*}\right)^{\Sigma(1)} \mid \prod_{\mathrm{p}} \mathrm{t}_{\mathrm{p}}^{\left\langle\mathrm{m}, \mathrm{u}_{\mathrm{p}}\right\rangle}=1, \text { para toda } \mathrm{m} \in \mathrm{M}\right\} \\
& =\left\{\left(\mathrm{t}_{\mathrm{p}}\right) \in\left(\mathrm{k}^{*}\right)^{\Sigma(1)} \mid \prod_{\mathrm{p}} \mathrm{t}_{\mathrm{p}}^{\left\langle\mathrm{e}_{\mathrm{i}}, \mathrm{u}_{\mathrm{p}}\right\rangle}=1, \quad 1 \leqslant \mathrm{i} \leqslant \mathrm{n}\right\} .
\end{aligned}
$$

Demostración. Iniciaremos demostrando el inciso $b$. Por la proposición 3.2 tenemos que $\mathrm{Cl}\left(\mathrm{X}_{\Sigma}\right)$ es un grupo abeliano finitamente generado, por lo que

$$
\mathrm{Cl}\left(\mathrm{X}_{\Sigma}\right) \simeq \mathbb{Z}^{\mathrm{l}} \times \mathrm{H}
$$

donde $\mathrm{H}$ es un grupo finito abeliano. Entonces

$$
\mathrm{G}=\operatorname{Hom}_{\mathbb{Z}}\left(\mathrm{Cl}\left(\mathrm{X}_{\Sigma}\right), \mathrm{k}^{*}\right) \simeq \operatorname{Hom}_{\mathbb{Z}}\left(\mathbb{Z}^{\mathrm{l}} \times \mathrm{H}, \mathrm{k}^{*}\right) \simeq\left(\mathrm{k}^{*}\right)^{\mathrm{l}} \times \operatorname{Hom}_{\mathbb{Z}}\left(\mathrm{H}, \mathrm{k}^{*}\right),
$$

como $\mathrm{H}$ es finito, $\operatorname{Hom}_{\mathbb{Z}}\left(\mathrm{H}, \mathrm{k}^{*}\right)$ es finito. 
Para el inciso $a$, sea $\chi(\mathrm{G})=\operatorname{Hom}\left(\mathrm{G}, \mathrm{k}^{*}\right)$ el grupo de caracteres de $\mathrm{G}$. Sea $\alpha \in \mathrm{Cl}\left(X_{\Sigma}\right)$, definimos $\lambda \in \chi(G)$ como $\lambda(g)=g(\alpha) \in k^{*}$, es decir, $\alpha$ nos define un caracter de $\mathrm{G}$. Ahora bien, aplicando el $\operatorname{Hom}_{\mathbb{Z}}\left(-, k^{*}\right)$ a la siguiente sucesión exacta

$$
1 \longrightarrow \mathrm{G} \longrightarrow\left(\mathrm{k}^{*}\right)^{\Sigma(1)} \longrightarrow \mathrm{T}_{\mathrm{N}} \longrightarrow 1
$$

tenemos

$$
1 \longrightarrow \mathrm{M} \longrightarrow \mathbb{Z}^{\Sigma(1)} \longrightarrow \chi(\mathrm{G}) \longrightarrow 1
$$

Dado que los puntos de $\mathbb{Z}^{\Sigma(1)}$ define un elemento $\alpha \in \mathrm{Cl}\left(\mathrm{X}_{\Sigma}\right)$ tenemos que todo elemento de $\chi(G)$ proviene de un $\alpha$. Por lo tanto $\chi(G) \simeq \mathrm{Cl}\left(X_{\Sigma}\right)$.

Para el último inciso recordemos que $\lambda \in \operatorname{Hom}_{\mathbb{Z}}\left(M, k^{*}\right)$ se define como la evaluación del caracter asociado a $m \in M$ es decir, $\lambda(m)=\chi^{m}(t)=t_{1} m_{1}$. $t_{2} m_{2} \cdots t_{n} m_{n}$, por lo tanto el morfismo identidad, $\lambda_{e}$ es $\lambda_{e}(m)=\chi^{m}(t)=$ $t_{1} m_{1} \cdot t_{2} m_{2} \ldots t_{n} m_{n}=1$ para toda $m \in M$. La misma caracterizacion se tiene para $\left(k^{*}\right)^{\Sigma(1)} \simeq \operatorname{Hom}_{\mathbb{Z}}\left(\mathbb{Z}^{\Sigma(1)}, k^{*}\right)$ por otro lado los puntos de $\mathbb{Z}^{\Sigma(1)}$ representan un elemento de $\mathrm{Cl}\left(\mathrm{X}_{\Sigma}\right)$ mediante la asignación $\left(\left\langle\mathrm{m}, \mathrm{u}_{\mathrm{p}}\right\rangle\right)$ donde $\mathrm{p} \in \Sigma(1)$, por lo tanto los elementos de $\mathrm{G} \subseteq\left(\mathrm{k}^{*}\right)^{\Sigma(1)}$ satisfacen la relación deseada, es decir

$$
G=\left\{\left(t_{p}\right) \in\left(k^{*}\right)^{\Sigma(1)} \mid \prod_{p} t_{p}^{\left\langle m, u_{p}\right\rangle}=1 \quad \text { para toda } m \in M\right\} .
$$

En particular si $\left\{e_{1}, \cdots, e_{n}\right\}$ es una base de $M$ se tiene que para todo $g=\left(t_{p}\right) \in G$ y para cada $e_{i}, \prod_{p} t_{p}\left\langle e_{i}, u_{p}\right\rangle=1$, cumpliendo así la segunda igualdad en el inciso $c$.

Definimos el siguiente ideal monomial en el anillo de coordenadas de $(k)^{\Sigma(1)}$, para cada $p \in \Sigma(1)$ asignemosle la variable $x_{p}$ y consideremos

$$
S=k\left[x_{p} \mid p \in \Sigma(1)\right]
$$

entonces el Specm $(S)=(k)^{\Sigma(1)}$. A $S$ se le denomina el anillo total de coordenadas de $X_{\Sigma}$. Ahora bien para cada $\sigma \in \Sigma$, definimos el monomio

$$
x^{\widehat{\sigma}}=\prod_{p \notin \sigma(1)} x_{p} .
$$

Consideremos el ideal

$$
\mathrm{B}(\Sigma)=\left\langle\chi^{\widehat{\sigma}} \mid \sigma \in \Sigma\right\rangle \subseteq \mathrm{S} .
$$

Notemos que si $x^{\widehat{\tau}}$ es un múltiplo de $\chi^{\widehat{\sigma}}$ cuando $\tau$ es cara $\sigma$.

Por lo que $\Sigma_{\max }=\{\sigma \in \Sigma \mid \sigma \quad$ es un cono máximo de $\quad \Sigma\}$ genera a $\mathrm{B}(\Sigma)$, es decir

Se define

$$
\mathrm{B}(\Sigma)=\left\langle\chi^{\widehat{\sigma}} \mid \sigma \in \Sigma_{\max }\right\rangle
$$

$$
\mathrm{Z}(\Sigma)=\mathrm{V}(\mathrm{B}(\Sigma)) \subseteq(\mathrm{k})^{\Sigma(1)} .
$$

Definición 3.26. Un subconjunto $C \subseteq \Sigma(1)$ es una colección primitiva si

(a). $C \nsubseteq \sigma(1)$ para todo $\sigma \in \Sigma$.

(b). Para cada subconjunto propio $C^{\prime} \subsetneq C$ existe $\sigma \in \Sigma$ con $C^{\prime} \subseteq \sigma(1)$. 
Con esta definición podemos dar una descripción de $Z(\Sigma)$.

Proposición 3.27. $\mathrm{Z}(\Sigma)$ como unión de componentes irreducibles está dada por

$$
\mathrm{Z}(\Sigma)=\bigcup_{\mathrm{C}} \mathrm{V}\left(\mathrm{x}_{\mathrm{p}} \mid \mathrm{p} \in \mathrm{C}\right)
$$

donde la unión es sobre todas las colecciones primitivas $C \subseteq \Sigma(1)$.

Demostración. Demostremos que los subespacios coordenados máximos contenidos en $Z(\Sigma)$ provienen de colecciones primitivas.

Supongamos que $V\left(x_{\rho_{1}}, \cdots, x_{\rho_{s}}\right)$ es un subespacio cumpliendo la hipótesis. Por lo tanto el ideal de anulación $\mathrm{I}=\left\langle\chi_{\rho_{1}}, \cdots, x_{\rho_{s}}\right\rangle$ es primo. Luego, por construcción, para cualquier cono $\sigma \in \Sigma$ se tiene que $x^{\widehat{\sigma}}$ se anula en todo $Z(\Sigma)$ por lo que existe un $x_{\rho_{i}}$ que divide a $\chi^{\widehat{\sigma}}$, es decir que la variable $x_{\rho_{i}}$ aparace en su expresión monomial por lo tanto el rayo asociado a $x_{\rho_{i}}$, no pertenece a $\sigma(1)$. Sea $C=\left\langle\rho_{1}, \cdots, \rho_{s}\right\rangle$ contenido en $\Sigma(1)$, demostramos que para cualquier cono $\sigma \in \Sigma(1)$ se cumple que $C \nsubseteq \sigma(1)$. Ahora bien, sea $C^{\prime}=C \backslash\left\{\rho_{i}\right\}$, basta demostrar que si todo subconjunto propio de esta forma está contenido en $\sigma(1)$ para algún cono, se cumpla que cualquier subconjunto propio de $C$, esté contenido en $\sigma(1)$ para algún $\sigma$. Por lo que consideremos $C^{\prime}$ como antes, entonces $\left\langle x_{\rho_{i}}\right\rangle \subsetneq\left\langle x_{\rho_{1}}, \cdots, x_{\rho_{s}}\right\rangle$ implica que

$$
\mathrm{V}\left(\mathrm{x}_{\rho_{1}}, \cdots, \mathrm{x}_{\rho_{\mathrm{s}}}\right) \subsetneq \mathrm{V}\left(\mathrm{x}_{\rho_{\mathrm{i}}}\right)
$$

dado que $\left\langle x_{\rho_{i}}\right\rangle$ es primo y por ser $\mathrm{V}\left(x_{\rho_{1}}, \cdots, x_{\rho_{s}}\right)$ máximo, se tiene que

$$
\mathrm{Z}(\Sigma) \subset \mathrm{V}\left(\mathrm{x}_{\rho_{\mathrm{i}}}\right)
$$

por lo que existe $\sigma \in \Sigma$ tal que $x^{\widehat{\sigma}}$ divide a $x_{\rho_{i}}$ lo cual implica que $x^{\widehat{\sigma}}=x_{\rho_{i}}$. Entonces $\rho_{i} \notin \sigma(1)$ pero por definición de $x^{\widehat{\sigma}}$ se tiene que $\left\{\rho \mid \rho \neq \rho_{i}\right\} \subset \sigma(1)$. En particular $C^{\prime}$ está contenido en $\sigma(1)$. Con lo que se demuestra que $C$ es una colección primitiva.

Ahora bien, sea $C=\left\{\rho_{1}, \cdots, \rho_{s}\right\}$ una colección primitiva, consideremos el ideal definido por las coordenadas asociadas a los rayos en $\mathrm{C}, \mathrm{I}_{\mathrm{C}}=\left\langle x_{\rho_{1}}, \cdots, x_{\rho_{s}}\right\rangle$. Por ser $C$ primitiva se tiene que para todo $\sigma \in \Sigma, C \nsubseteq \sigma(1)$ por lo que existe $\rho_{i}$ tal que no pertenece a $\sigma(1)$ por lo tanto $x_{\rho_{i}}$ divide a $x^{\widehat{\sigma}}$. Lo cual implica que para todo cono $\sigma \in \Sigma$ se tiene que $x^{\widehat{\sigma}}$ se anula en $V\left(\mathrm{I}_{\mathrm{C}}\right)$ concluyendo que $\mathrm{V}\left(\mathrm{I}_{\mathrm{C}}\right) \subseteq \mathrm{Z}(\Sigma)$.

Supongamos que existe $\mathrm{V}$ tal que $\mathrm{V}\left(\mathrm{I}_{\mathrm{C}}\right) \subsetneq \mathrm{V}$. Sea I el ideal de anulación de $\mathrm{V}$. Entonces por condiciones de contención I $\subsetneq I_{C}$, por lo que $\mathrm{I}=\left\langle x_{\rho_{\mathrm{t}_{1}}}, \cdots, x_{\rho_{\mathrm{t}_{1}}}\right\rangle$. Sea $C^{\prime}=\left\{\rho_{t_{1}}, \cdots, \rho_{t_{l}}\right\} \subsetneq C$, por lo que existe un cono tal que $C^{\prime} \subseteq \sigma(1)$. Considere $x^{\widehat{\sigma}}=\prod_{\rho \notin \sigma(1)} x_{\rho}$, entonces $x_{\rho_{t_{i}}}$ no divide a $x^{\widehat{\sigma}}$. Es decir, existe un cono $\sigma$ tal que $x^{\widehat{\sigma}}$ no se anula en $V$, por lo tanto $V \nsubseteq Z(\Sigma)$.

Observación 3.28. Dado que $\left(k^{*}\right)^{\Sigma(1)}$ actúa en $(k)^{\Sigma(1)}$ por medio de las matrices diagonales, se tiene que $\left(k^{*}\right)^{\Sigma(1)}$ actúa en $(k)^{\Sigma(1)} \backslash Z(\Sigma)$. Por lo tanto $G \subseteq$ $\left(k^{*}\right)^{\Sigma(1)}$ también actúa en $(k)^{\Sigma(1)} \backslash Z(\Sigma)$. 
Para poder demostrar que toda variedad tórica es casi un buen cociente requerimos de una variedad afín adecuada, la cual será construidad a partir de un nuevo abanico. Sea $X_{\Sigma}$ una variedad afín tórica con abanico $\Sigma \subseteq N_{\mathbb{R}}$. Consideremos la $\mathbb{Z}$ base estándar de $\mathbb{Z}^{\Sigma(1)}$

$$
\left\{e_{p} \mid p \in \Sigma(1)\right\} .
$$

Para cada $\sigma \in \Sigma$ definimos el siguiente cono

$$
\tilde{\sigma}=\operatorname{cono}\left(e_{p} \mid p \in \sigma(1)\right) \subseteq \mathbb{R}^{\Sigma(1)} .
$$

Dado que los conos que conforman nuestro abanico $\Sigma$ son conos poliédricos fuertemente convexos, podemos construir el siguiente abanico

$$
\tilde{\Sigma}=\{\tau \mid \tau \preceq \tilde{\sigma} \text { para algún } \quad \sigma \in \Sigma\}
$$

en $\left(\mathbb{Z}^{\Sigma(1)}\right)_{\mathbb{R}}=\mathbb{R}^{\Sigma(1)}$. Este nuevo abanico tiene las siguientes propiedades.

Proposición 3.29. Sea $\tilde{\Sigma}$ como antes. Entonces:

(a). $(k)^{\Sigma(1)} \backslash Z(\Sigma)$ es la variedad tórica del abanico $\tilde{\Sigma}$.

(b). La aplicación $\mathrm{e}_{\mathrm{p}} \longrightarrow \mathrm{u}_{\mathrm{p}}$ define un morfismo entre las retículas $\mathbb{Z}^{\Sigma(1)} \longrightarrow$ $\mathrm{N}$ compatible con los abanicos $\tilde{\Sigma} y \Sigma$.

(c). El morfismo tórico inducido

$$
\pi:(k)^{\Sigma(1)} \backslash \mathrm{Z}(\Sigma) \longrightarrow \mathrm{X}_{\Sigma}
$$

es constante en órbitas.

Demostración. Consideremos el siguiente abánico, $\Sigma_{0}$ formado por

$$
\operatorname{cono}\left(e_{\rho} \mid \rho \in \Sigma(1)\right)
$$

y sus caras, el cual es el abanico asociado a la variedad tórica $(k)^{\Sigma(1)} \cdot \tilde{\Sigma}$ es subabanico de $\Sigma_{0}$, aplicando el teorema de correspondencia entre órbitas y conos (3.19) en una variedad tórica, se tiene que la variedad tórica $X_{\Sigma}$ se obtiene de $(k)^{\Sigma(1)}$ al remover las órbitas correspondientes a los conos en $\Sigma_{0} \backslash \tilde{\Sigma}$, es decir, la cerradura de las órbitas de conos mínimos. Ahora bien, todo cono en $\tilde{\sigma} \in \Sigma_{0} \backslash \tilde{\Sigma}$ es tal que $\tilde{\sigma} \notin \sigma(1)$ para todo cono en $\tilde{\Sigma}$, a su vez, $\tilde{\sigma}$ debe ser un cono mínimo, es decir, cualquier subconjunto propio de $\tilde{\sigma}(1)$ está contenido en $\sigma(1)$ para algún cono de $\tilde{\Sigma}$. Es decir, hay que remover los conos asociados a las colecciones primitivas de $\tilde{\Sigma}$. De nuevo por el teorema de correspondecia de órbitas y conos esto es equivalente a eliminar para cada colección primitiva

$$
\mathrm{V}\left(x_{\rho} \mid \rho \in \mathrm{C}\right) \text {, }
$$

por lo tanto eliminar todos las órbitas asociadas a los conos en $\Sigma_{0} \backslash \tilde{\Sigma}$ es equivalente a eliminar

$$
Z(\Sigma)=\bigcup_{C} V\left(x_{\rho} \mid \rho \in C\right)
$$

Concluyendo así la demostración del inciso a).

Recordemos que la retícula asociada a $X_{\Sigma}$ es $N$, entonces definimmos el morfismo entre retículas:

$$
\bar{\pi}: \mathbb{Z}^{\Sigma(1)} \longrightarrow \mathrm{N}
$$


definida por $\bar{\pi}\left(e_{\rho}\right)=u_{\rho}$ (el rayo mínimo en $N$ ). Dado que cada cono $\sigma$ de $\mathbb{Z}^{\Sigma(1)}$ está generado por $u_{\rho}$ con $\rho \in \sigma(1)$ se tiene que $\bar{\pi}_{\mathbb{R}}(\tilde{\sigma})=\sigma$ por construcción de $\tilde{\Sigma}$. Por lo tanto $\pi$ es un morfismo compatible con los abanicos $\Sigma$ y $\tilde{\Sigma}$

Ahora bien, el morfismo $\bar{\pi}: \mathbb{Z}^{\Sigma(1)} \longrightarrow \mathrm{N}$ induce un morfismo entre las retículas duales correspondientes, $M$ y $\mathbb{Z}^{\Sigma(1)}$, es decir, tomar el funtor $\operatorname{Hom}_{\mathbb{Z}}\left(-, k^{*}\right)$ es decir, el morfismo de toros inducido por $\bar{\pi}$ coincide con el morfismo de toros de la sucesión exacta corta (6). Dado que $G \subseteq\left(k^{*}\right)^{\Sigma(1)}$ se tiene que para todo $g \in G$ y $x \in(k)^{\Sigma(1)} \backslash Z(\Sigma)$, se tiene

$$
\bar{\pi}(g x)=\bar{\pi}(g) \bar{\pi}(x)=\bar{\pi}(x)
$$

la primera igualdad se debe a que $\bar{\pi}$ es el morfimos correspondiente entre toros y por lo tanto se aplica la equivarianza, y la segunda porque $\mathrm{G}$ está contenido en el núcleo del morfimos entre los toros de la sucesión exacta corta. Demostrando que $\bar{\pi}$ es constante en órbitas.

Para finalizar el capítulo daremos la demostración de que toda variedad tórica es un casi buen cociente, pero este resultado será hecho para el caso de que el campo es el de los números complejos. Requererimos un lema previo.

Lema 3.30. Suponga que $V$ es una variedad tórica afín no necesariamente normal, con toro $\mathrm{T}$. Dado un punto $\overline{\mathrm{p}} \in \mathrm{V}$ existe un punto $\overline{\mathrm{q}} \in \mathrm{T}$ y un subgrupo a un parámetro $\lambda: \mathbb{C}^{*} \longrightarrow \mathrm{T}$ tal que

$$
\overline{\mathrm{p}}=\lim _{\mathrm{t} \rightarrow 0} \lambda(\mathrm{t}) \overline{\mathrm{q}} .
$$

Teorema 3.31. Sea $X_{\Sigma}$ una variedad tórica sin factor toro y considere el morfismo tórico $\pi:(\mathbb{C})^{\Sigma(1)} \backslash Z(\Sigma) \longrightarrow X_{\Sigma}$ de la proposición 3.29 anterior. Entonces

(a). $\pi$ es un casi buen cociente geométrico para la acción de $\mathrm{G}$ en $(\mathbb{C})^{\Sigma(1)} \backslash Z(\Sigma)$. Por lo tanto

$$
\mathrm{X}_{\Sigma} \simeq(\mathbb{C})^{\Sigma(1)} \backslash \mathrm{Z}(\Sigma) / / \mathrm{G} \text {. }
$$

(b). $\pi$ es un cociente geométrico si y sólo si $\Sigma$ es simplicial.

Demostración. Sea $\sigma \in \Sigma$, consideremos el morfismo

$$
\pi_{\mid \pi^{-1}\left(\mathrm{U}_{\sigma}\right)}: \pi^{-1}\left(\mathrm{U}_{\sigma}\right) \longrightarrow \mathrm{U}_{\sigma} .
$$

Denotemos por $\pi_{\sigma}$ a $\pi_{\mid \pi^{-1}\left(\mathrm{U}_{\sigma}\right)}$. Por construcción del morfismo $\pi$, se tiene que para todo $\tau, \sigma \in \Sigma$ si $\bar{\pi}_{\mathbb{R}}(\tilde{\tau}) \subset \sigma$ implica que $\tau \preceq \sigma$. Por lo tanto $\pi^{-1}\left(\mathrm{U}_{\sigma}\right)$ es la variedad tórica $U_{\tilde{\sigma}}$ asociada al cono $\tilde{\sigma}=\operatorname{cono}\left\{e_{p} \mid p \in \sigma(1)\right\}$. Es decir tenemos el siguiente morfismo tórico

$$
\pi_{\sigma}: \mathrm{U}_{\tilde{\sigma}} \longrightarrow \mathrm{U}_{\sigma} \text {. }
$$

Dado que $\mathrm{G}$ es un grupo reductivo, por el teorema 2.29, basta demostrar que $\pi_{\sigma}^{*}$ induce un isomorfismo entre los anillos de coordenadas

$$
\mathbb{C}\left[\mathrm{U}_{\tilde{\sigma}}\right] \simeq \mathbb{C}\left[\mathrm{U}_{\sigma}\right]^{\mathrm{G}}
$$

para que $\pi_{\sigma}$ sea un buen cociente categórico.

Analicemos los anillos coordenados involucrados 
- Para $\mathrm{U}_{\tilde{\sigma}}$, el semigrupo asociado al cono $\tilde{\sigma}$ es

$$
\tilde{\sigma}^{\vee} \cap \mathbb{Z}^{\Sigma(1)}=\left\{\left(a_{\rho}\right) \in \mathbb{Z}^{\Sigma(1)} \mid a_{\rho} \geqslant 0 \text { para todo } \rho \in \sigma(1)\right\} .
$$

Entonces el anillo de coordenadas es

$$
\mathbb{C}\left[\mathrm{U}_{\tilde{\sigma}}\right]=\left[\prod_{\rho} x_{\rho}^{\mathrm{a}_{\rho}} \mid \mathrm{a}_{\rho} \geqslant 0 \quad \text { para todo } \rho \in \sigma(1)\right]=\mathrm{S}_{\chi^{\widehat{\sigma}}}
$$

donde $S_{x^{\widehat{\sigma}}}$ es la localización de $S=\mathbb{C}\left[x_{\rho} \mid \rho \in \Sigma(1)\right]$ en $x^{\widehat{\sigma}}=\prod_{\rho \notin \sigma(1)} x_{\rho}$.

- Para $U_{\sigma}$ su anillo de coordenadas es $\mathbb{C}\left[\sigma^{\vee} \cap M\right]$.

- El morfismo $\bar{\pi}: \mathrm{N} \longrightarrow \mathbb{Z}^{\Sigma(1)}$ tiene por dual el morfismo de la sucesión exacta corta (5) como se demostró previamente, es decir, el morfismo $M \longrightarrow \mathbb{Z}^{\Sigma(1)}$ definido por $\bar{\pi}^{\vee}(\mathrm{m})=\left(\left\langle\mathrm{m}, \mathrm{u}_{\rho}\right\rangle\right) \in \mathbb{Z}^{\Sigma(1)}$. Dado que los caracteres del toro $T_{N}$ son una base para su anillo de coordenadas, basta analizar el morfismo entre los anillos coordenadas, $\pi_{\sigma}{ }^{*}: \mathbb{C}\left[\sigma^{\vee} \cap M\right] \longrightarrow$ $\mathrm{S}_{\chi^{\hat{\sigma}}}$ en los caracteres, es decir

$$
\pi_{\sigma}^{*}\left(\chi^{m}\right)=\prod_{\rho} x_{\rho}^{\left\langle m, u_{\rho}\right\rangle}
$$

pero este morfismo está bien definido puesto que $\left\langle\mathrm{m}, \mathrm{u}_{\rho}\right\rangle \geqslant 0$ para todo $\rho \in \sigma(1)$ por definición de su semigrupo asociado.

Por otro lado $\pi_{\sigma}$ es constante en órbitas, se tiene en realidad que

$$
\pi^{*}{ }_{\sigma}: \mathbb{C}\left[\sigma^{\vee} \cap M\right] \longrightarrow\left(S_{\chi^{\widehat{\sigma}}}\right)^{\mathrm{G}} \text {. }
$$

De la sucesión exacta corta (6) se tiene que $\pi_{\sigma}\left((\mathbb{C})^{\Sigma(1)}\right)=\mathrm{T}_{\mathrm{N}}$ entonces, la imagen de $\mathrm{U}_{\tilde{\sigma}}$ bajo $\pi_{\sigma}$ contiene un abierto denso, por lo tanto $\pi^{*}{ }_{\sigma}$ es un morfismo inyectivo. Demostremos que $\pi^{*}{ }_{\sigma}$ es suprayectivo, sea $f \in S_{\chi \widehat{\sigma}}$, entonces $f$ tiene la siguiente expresión

$$
f=\sum_{a} c_{a} x^{a}
$$

donde $x^{a}=\prod_{\rho} x_{\rho}^{a_{\rho}} \in \mathbb{C}\left[U_{\tilde{\sigma}}\right]$, luego $f$ es $G$-invariante si y sólo si para todo $t=\left(t_{\rho}\right) \in G$ se tiene que

$$
\sum_{a} c_{a} x^{a}=\sum_{a} c_{a} t^{a} x^{a} .
$$

$O$ bien, $f$ es $G$-invariante si y sólo si $t^{a}=1$ para toda $t \in G$ siempre que $c_{a} \neq 0$. Observe que la aplicación $t \rightarrow t^{a}$ es un caracter de $G$ por tanto pertenece a su grupo de caracteres, es decir a $\mathrm{Cl}\left(\mathrm{X}_{\Sigma}\right)$. Dado que este caracter es trivial cuando $c_{a} \neq 0$ se tiene por la sucesión exacta corta (5) que para el exponente $a=\left(a_{\rho}\right)$ existe $m \in M$ tal que $a_{\rho}=\left\langle m, u_{\rho}\right\rangle$ para $\rho \in \Sigma(1)$. Por otro lado, se tiene que $x^{a} \in S_{x^{\widehat{\sigma}}}$ por lo que

$$
a_{\rho}=\left\langle m, u_{\rho}\right\rangle \geqslant 0 \text { para todo } \rho \in \Sigma(1) .
$$

Demostrando que $m \in \sigma^{\vee} \cap M$ lo cual a su vez implica que $f$ pertenece a la imagen de $\pi_{\sigma}^{*}$. Concluyendo así que $\pi_{\sigma}$ es un buen cociente categórico. 
Para la siguiente parte de la prueba, supongamos primero que $\sigma$ es simplicial. La prueba se reduce a demostrar que las $\mathrm{G}$ órbitas son cerradas en $\mathrm{U}_{\tilde{\sigma}}$ por el teorema 2.27 del capítulo 2. Además por la caracterización de las componentes conexas de un grupo algebraico afiín, en este caso $\mathrm{G}$, basta probar que las $\mathrm{G}_{\mathrm{e}}$-órbitas, de la componente conexa que contiene a la identidad del grupo, son cerradas en $\mathrm{U}_{\tilde{\sigma}}$, puesto que $\mathrm{G}_{e}$ es de índice finito en $\mathrm{G}$.

Sea $p \in \mathrm{U}_{\tilde{\sigma}} \mathrm{y} \overline{\mathrm{p}} \in \overline{\text { orb }_{\mathrm{G}}(\mathrm{p})} \subset \mathrm{U}_{\tilde{\sigma}}$. Observe que órb $\mathrm{or}_{\mathrm{G}}(\mathrm{p})$ es una variedad tórica (posiblemente no normal) con toro $\mathrm{T} \simeq \mathrm{G}_{e} /\left(\mathrm{G}_{e}\right)_{\mathrm{p}}$. Por el lema 3.30 previo a este teorema, existe un subgrupo a un parámetro, $\lambda^{\prime}: \mathbb{C} \longrightarrow \mathrm{T}$ y q $\mathrm{q}^{\prime} \in \mathrm{T}$ tal que

$$
\overline{\mathrm{p}}=\lim _{\mathrm{t} \rightarrow 0} \lambda^{\prime}(\mathrm{t}) \mathrm{q}^{\prime} \cdot \mathrm{p} .
$$

Este subgrupo a un parámetro define un subgrupo a un parámetro $\lambda: \mathbb{C} \longrightarrow \mathrm{G}_{e} \mathrm{y}$ un punto $\mathrm{q} \in \mathrm{G}_{e}$ tal que

$$
\bar{p}=\lim _{t \rightarrow 0} \lambda(t) q \cdot p .
$$

Ahora bien, dado que $G_{e} \subset\left(\mathbb{C}^{*}\right)^{\Sigma(1)}$ podemos escribir $\lambda(t)=\left(t^{a_{\rho}}\right)$ con $a_{\rho} \in \mathbb{Z}$. De hecho, por el lema 3.25 se cumple que

$$
\sum_{\rho} a_{\rho} u_{\rho}=0
$$

Escribamos a $p=\left(p_{\rho}\right), \bar{p}=\left(\bar{p}_{\rho}\right)$ y $q=\left(q_{\rho}\right)$, analizando el límite en cada componente, tenemos

$$
\bar{p}_{\rho}=\lim _{t \rightarrow 0} \lambda\left(t^{a_{\rho}}\right) q_{\rho} \cdot p_{\rho} .
$$

Dado que $p, \bar{p} \in U_{\tilde{\sigma}}$ y $q \in G_{e}$, se tiene que sus $\rho$-ésimas componentes son no ceros para $\rho \notin \sigma(1)$. Entonces, el límite en cada una de esas componentes para que esté bien definido se necesita que $a_{\rho}=0$, por lo que tenemos la siguiente condición

$$
\sum_{\rho \in \sigma(1)} a_{\rho} u_{\rho}=0
$$

Sin embargo, $\sigma$ es simplicial, lo cual implica que $u_{\rho}$ con $\rho \in \sigma(1)$ son linealmente independientes. Entonces $a_{\rho}=0$ para todo $\rho \in \sigma(1)$. En resumen $a_{\rho}=0$ para todo $\rho$. Demostrando que $\lambda$ es el subgrupo a un parámetro trivial. Concluyendo así que $\bar{p}$ pertenece a órb $\mathrm{G}_{e}(p)$, es decir, la órbita es cerrada en $\mathrm{U}_{\tilde{\sigma}}$.

Para demostrar la otra implicación, supongamos que $\sigma \in \Sigma$ no es simplicial. Entonces existe una relación

$$
\sum_{\rho \in \sigma(1)} a_{\rho} u_{\rho}=0
$$

donde $a_{\rho} \in \mathbb{Z}$ y al menos un $a_{\rho}>0$. Definamos $a_{\rho}=0$ para $\rho \notin \sigma(1)$. Esto permite definir un subgrupo a un parámetro

$$
\lambda(t)=\left(t^{a_{\rho}}\right) \in\left(\mathbb{C}^{*}\right)^{\Sigma(1)},
$$


el cual también es un subgrupo a un parámetro de G. La razón de ello es que, para toda $m \in \mathbb{Z}^{\Sigma(1)}$ se tiene que

$$
\begin{aligned}
& \lambda(\mathrm{t})=\left(\mathrm{t}^{\mathrm{a}_{\rho}}\right) \in \mathrm{G} \Longleftrightarrow \prod_{\rho}\left(\mathrm{t}^{\mathrm{a}_{\rho}}\right)^{\left\langle\mathrm{m}, \mathrm{u}_{\rho}\right\rangle}=1 \\
& \Longleftrightarrow \prod_{\rho}(\mathrm{t})^{\mathrm{a}_{\rho}\left\langle\mathrm{m}, \mathrm{u}_{\rho}\right\rangle}=1 \\
& \Longleftrightarrow \prod_{\rho}(\mathrm{t})^{\left\langle\mathrm{m}, \mathrm{a}_{\rho} \mathrm{u}_{\rho}\right\rangle}=1 \\
& \Longleftrightarrow(t)^{\sum_{\rho}\left\langle m, a_{\rho} u_{\rho}\right\rangle}=1 \\
& \Longleftrightarrow(t)^{\left\langle m, \Sigma_{\rho} a_{\rho} u_{\rho}\right\rangle}=1 \\
& \Longleftrightarrow \sum_{\rho} a_{\rho} u_{\rho}=0 \text {. }
\end{aligned}
$$

Ahora bien, definimos un punto $p=\left(p_{\rho}\right) \in U_{\tilde{\sigma}}$ por

$$
p_{\rho}= \begin{cases}1 & a_{\rho} \geqslant 0 \\ 0 & a_{\rho}<0 .\end{cases}
$$

Ahora bien, considere el $\lim _{t \rightarrow 0} \lambda(t) p$, este límite existe en $(\mathbb{C})^{\Sigma(1)}$ ya que $p_{\rho}=0$ para $a_{\rho}<0$. De hecho, si $\rho \notin \sigma(1)$ se tiene que $a_{\rho}=0$ por lo $\rho$-ésima componente de $\lambda(t) p$ es igual a 1 para toda $t$, por lo que $\bar{p}=\operatorname{lím}_{t \rightarrow 0} \lambda(t) p$ pertenece a $\mathrm{U}_{\tilde{\sigma}}$. Por la condición de dependencia lineal, tenemos que existe $\rho_{0} \in \sigma(1)$ tal que $a_{\rho_{0}}>0$, entonces

- Dado que $\rho_{0}$-ésima entrada de $p$ es distinto de cero, lo mismo para todo $g p \in \operatorname{orb}_{\mathrm{G}}(\mathrm{p})$

- La condición que $a_{\rho_{0}}>0$ implica que $\rho_{0}$-ésima entrada de

$$
\overline{\mathrm{p}}=\lim _{\mathrm{t} \rightarrow 0} \lambda(\mathrm{t}) \mathrm{p}
$$

es cero.

Demostrando así que existe un punto $\bar{p} \in \overline{\text { órb }_{\mathrm{G}}(p)} \backslash$ órb $_{\mathrm{G}}(\mathrm{p})$, con lo cual se concluye que la órbita no es cerrada en $\mathcal{U}_{\tilde{\sigma}}$. Finalizando así de que $\pi_{\sigma}$ no es un buen cociente geométrico.

Para concluir con la demostración del teorema, usaremos los teoremas 2.27 y 2.28 del capítulo 2. Dado que $\pi_{\sigma}$ es un buen cociente categórico para cada $\sigma \in \Sigma$ se tiene que:

$$
\pi:(\mathbb{C})^{\Sigma(1)} \backslash Z(\Sigma) \longrightarrow X_{\Sigma}
$$

también lo es.

Ahora bien, para probar el primer inciso del teorema, considere el subabanico que contiene a todos los conos simpliciales, denotémoslo por $\Sigma^{\prime} \subseteq \Sigma$. Entonces $X_{\Sigma}$ es abierto en $X_{\Sigma}$, además $\Sigma^{\prime}(1)=\Sigma(1)$, por lo que $X_{\Sigma}$, y $X_{\Sigma}$ poseen el mismo anillo total de coordenadas y el mismo grupo G. Al definir el abanico $\tilde{\Sigma}^{\prime} \subset \mathbb{Z}_{\mathbb{R}}^{\Sigma(1)}$ se tiene que 
- Para cada $\sigma \in \Sigma^{\prime}$ se tiene que $\pi_{\sigma}: \pi^{-1}\left(\mathrm{U}_{\sigma}\right)=\mathrm{U}_{\tilde{\sigma}}$ por lo que

$$
\pi^{-1}\left(\mathrm{X}_{\Sigma^{\prime}}\right)=\bigcup_{\sigma \in \Sigma^{\prime}}\left(\mathrm{U}_{\tilde{\sigma}}\right)
$$

- Se realiza el mismo análisis que en la proposición 3.29, sea $\Sigma_{0}$ como en ese caso, y $X_{\Sigma}$, es la variedad tórica resultante de remover la cerradura de conos minimos de $\Sigma_{0} \backslash \Sigma^{\prime}$ es decir, remover los conos cuyos rayos son linealmente dependientes, y como deben ser conos mínimos cualquier subconjunto propio de sus rayos debe ser un conjunto linealmente independiente, por lo tanto

$$
\pi^{-1}\left(X_{\Sigma^{\prime}}\right)=\bigcup_{\sigma \in \Sigma^{\prime}}\left(\mathrm{U}_{\tilde{\sigma}}\right)=(\mathbb{C})^{\Sigma(1)} \backslash Z\left(\Sigma^{\prime}\right) .
$$

Por las propiedades de ser un buen cociente categórico se tiene que

$$
\pi_{\mid \pi^{-}\left(\mathrm{X}_{\Sigma^{\prime}}\right)}: \pi^{-1}\left(\mathrm{X}_{\Sigma^{\prime}}\right) \longrightarrow \mathrm{X}_{\Sigma^{\prime}}
$$

es un buen cociente categórico y para cada $\sigma \in \Sigma^{\prime}, \pi_{\sigma}$ es un buen cociente geométrico, por lo tanto, $\pi$ es un casi buen cociente geométrico y es buen cociente geoétrico cuando $\Sigma$ es simplicial. Por último, si cada $\sigma \in \Sigma$ es simplicial, cada $\pi_{\sigma}$ es un buen cociente geométrico, de nuevo por los teoremas 2.27 y 2.28 del capítulo $2, \pi$ es un buen cociente geométrico. Quedado así demostrado este teorema. 



\section{Bibliografía}

[1] Cox, D., J. Little and H. Schenck, Toric Varieties. AMS, Providence, 2012.

[2] Cox, D. The homogeneous cordinate ring of a toric variety. J. Algebraic Geometry 4(1), 17-50 (1995). Errata, J. Algebraic Geometry 23 (2014) 393-398.

[3] Danilov, V., "The Geometry of Toric Varieties", Russian Mathematical Surveys 33 (1978), 97154.

[4] Dolgachev, I., Lectures on Invariant Theory. Cambridge University Press, London, 2003.

[5] Ferrer, W. and A. Rittatore, Actions and Invariants of Algebraic Groups. Chapman and Hall CRC Press, Boca Raton, 2005.

[6] Fulton, W., Introduction to Toric Varieties. Princeton University Press, Princeton, 1993.

[7] Hartshorne, R., Algebraic Geometry. Springer Verlag, Berlin, 1977.

[8] Humphreys, J., Linear Algebraic Groups. Springer Verlag, New York, 1975.

[9] Oda, T., Convex Bodies and Algebraic Geometry. Springer Verlag, Berlin, 1996.

[10] Ruiz, Juan., Secciones globales en variedades tóricas. Tesis de Maestría, 2013.

[11] Shafarevich, I. R., Basic Algebraic Geometry. Springer Verlag, Berlin, 1974.

[12] Zaldívar, F., Introducción a la geometría algebraica. 2010-2015. 